Gifted Individuals with Asperger's:

A Discourse Exploration of 'Being an Aspie'

\title{
Camellia Wong
}

A thesis submitted to the Victoria University of Wellington in partial fulfilment of the requirements for the degree of Master of Education

Victoria University of Wellington

2009 
$\sim 2 \sim$ 


\section{Abstract}

People labelled as both 'gifted' and as having Asperger's Syndrome are a social paradox. Asperger's is often associated with impairment and disability, and a dualistic 'opposite' of giftedness on a learning ability spectrum. This study explored life experiences of adults who self-identified as 'aspies' (a casual term used by people who are proud to have Asperger's as an identity) and who were also identified as being gifted in the widest sense. A literature review discovered few studies on the topic, and, of those found, most employed traditional medical explanations of the condition or 'syndrome'. This study adopted qualitative participatory methodology using interviews. Three men and one woman who identified as aspies participated in semi-structured interviews that were video recorded in order to better understand their communication strategies. A type of discourse analysis based on Foucault's use of discourse (Parker, 1992) was used to analyse their worldviews about Asperger's, giftedness and abilities. The analysis revealed an interplay of medical and other related discourses surrounding Asperger's as impairment, with a lesser emphasis on discourses of giftedness. Participants often felt marginalised due to stereotypes about 'incompetence' regarding people with Asperger's; a view seen as denigrating their talents. The main finding was that aspie identities were not always strong due to a conflicting need to 'fit in' and accommodate to 'normal' society. Their talents sometimes benefited their attempts at 'pretending to be normal'; however this depended on whether the skill was regarded by society as worthy.

Decisions to be proud aspies and resist social norms, sometimes had 
consequences of isolation, confusion, being misunderstood or judged as disabled. Whilst participants preferred to be accepted as aspies with talented skills, they found this social positioning often contradictory. The thesis ends with a suggestion that future research could adopt more participatory focuses, to enable more insight into ways that people labelled as having Asperger's and with giftedness discursively describe their worlds and concepts of ability. 
$\sim 5 \sim$ 
$\sim 6 \sim$ 


\section{Acknowledgements}

My journey of discovering what it means to be an aspie happened because I had a supportive, encouraging and creative team. This team followed me through the academic adventure that is a thesis: preparing ethical essentials, travelling through hurdles of literature, discovering participants, exploring data, voyaging though analysis, endless editing, and keeping me on track. They also showed me that there is light at the end of the tunnel. I hope my relationships with each of you grow from beyond this one journey.

First and foremost I must acknowledge my academic supervisor, Dr Lise Bird Claiborne. Lise, you believed in me and remained my constant role model and teacher. My way of thinking of the world has changed as you showed me how to think ingeniously. You taught me to reflect on myself as a researcher and I am humbled to have been your student. Kay, Kea, Eion and Alan, your words and wisdom will live on from this one thesis. Thank you for being advocates, participants and just being you. Finally, my partner Benny Yuen - you have always been a support and my 'rock' though life challenges, emotional and academic. You believed in me, and I cannot express by words how much that has meant. In addition to those already mentioned, I would like to thank Debra and Brie from Victoria University of Wellington Student Learning Support, Warren Butcher for creating my narratives into movies, Maureen Walker and Autism New Zealand, David Corner, Sue Cornforth, and the New Zealand Federation of Graduate Women (Wellington branch). 
Abstract.

Acknowledgements.

Chapter 1: 'Asperger's' - the gift of being uncommon.................. 13

Aims of the study ...................................................... 17

Research questions................................................. 20

Researcher background and interest to the study ..................... 20

The journey of aspies' narratives, chapter by chapter..................... 21

Chapter 2: Foundation before the journey............................... 25

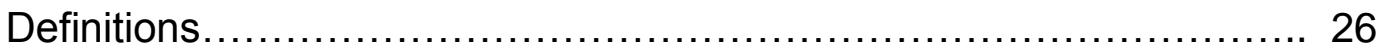

Intellectual impairment ............................................ 27

Autism spectrum and Asperger's syndrome......................... 32

Personal identity of 'aspie'......................................... 37

Interplay between Asperger's and Savant Syndrome............. 38

Disability ....................................................... 41

Giftedness....................................................... 43

A discursive framework for exploring the 'asperger' world ................ 51

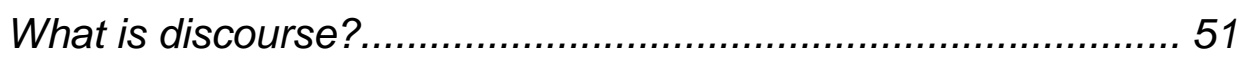

How is discourse used by people................................. 53

Who is Michel Foucault............................................. 54

Why is discourse used in my research about 'gifted aspies'?....... 56

Power and discourse .............................................. 56

Language, communication and discourse .......................... 59

Categories of disability discourse ............................... 61

Chapter 3 Veiled Genius: Manifesting inability, masking talents..... 69

Chapter 4 Life as we see it: 'Gifted aspies”' perceptions of life...... 79 The power of discursive communication and language for socialization. 82 Medical models and discourses....................................... 84 
Being part of a research community: participants and researchers working together 91

Research process that are disabling to participants.............. 94

Benefits for participants with 'asperger's' and giftedness........... 97

Beginning the journey of methodology............................. 99

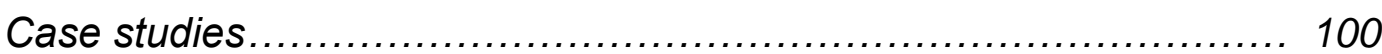

Gaining Entry and seeking participants.................... 101

Participant selection................................................. 104

Establishing rapport with research participants................. 106

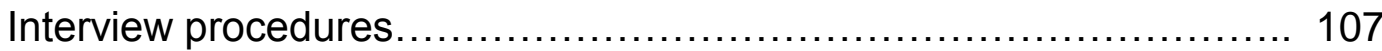

Trustworthiness between researcher and participants................ 112

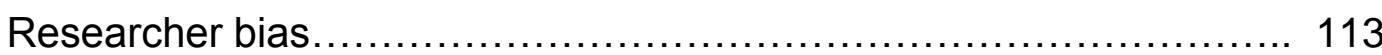

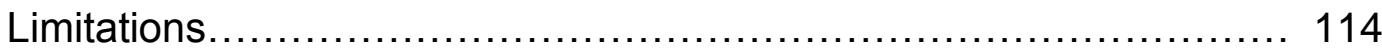

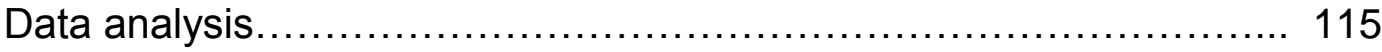

Chapter 6 “" Aspie’ is who I am”........................................119

Feeling different to other humans.................................. 119

Pathway to 'aspie' .............................................. 121

Diagnosis of 'aspergers' ................................................... 124

"Being aspie is optimistically unique:............................. 134

Chapter 7 Sharing the 'asperger' world as 'aspies'................... 139

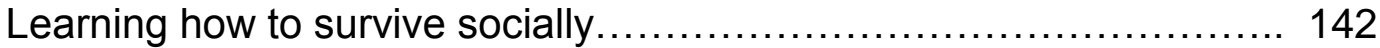

Positive advice to other 'aspies' .................................................. 143

Pretending to be 'normal' ...................................... 146

Chapter 8 Language and words in an 'asperger world'............. 149

Measurable 'aspergers' and talents.................................. 149

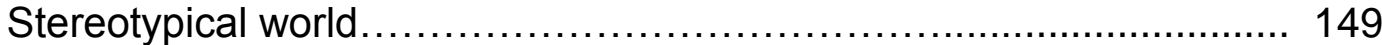

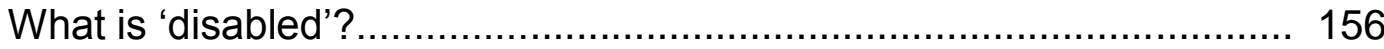

Continuing with categories, characters and classifications............ 157

Chapter 9 Gifted 'aspies' 
“I am an 'aspie' but am I gifted?" ............................... 161

"My 'asperger's' gives me abilities"............................... 163

Worthy abilities, talents and gifts............................... 167

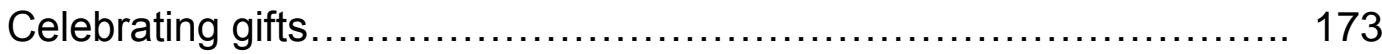

Chapter 10 Sharing an 'aspie' journey........................... 177

Influences of discourses about 'aspergers'.......................... 177

Living in a dualistic world....................................... 179

Hide the 'aspie' ... rather than pride of 'aspie' ......................... 181

Positioning oneself amongst an 'aspie' and normal communities....... 191

Chapter 11 Conclusion................................................... 188

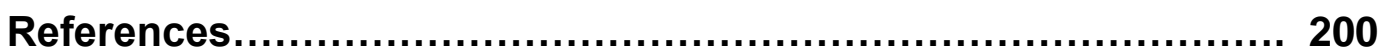

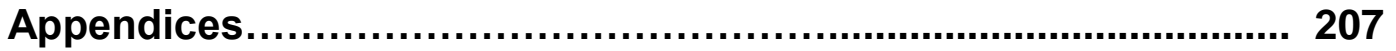


$\sim 11 \sim$ 
$\sim 12 \sim$ 


\section{Chapter 1}

\section{'Asperger's' - The Gift of Being Uncommon}

The life experiences of people who live with the labels 'gifted' and Asperger's Syndrome are unique and are a paradox. This thesis focuses on the conjunction of the two in the lives of people who have both talents and characteristics of Asperger's ${ }^{1}$. There may be a common belief that talent is demonstrated through academic, social or physical competence; conversely, anyone labelled as having aspergers is viewed as having a medical 'syndrome' - hence, a 'disability', which is by common definition, the binary opposite of 'higher ability' (Fulcher, 1989). A person who is socially categorized as having both the contrasting labels is in a paradoxical position, a mystery to people within their society and perhaps, to themselves. Termed 'dual exceptionalities' by Cash (1999, p.95), this anomaly is often because disability and giftedness are seemingly on either extremes of a social 'learning ability spectrum', outside usual cultural norms (MacFarlane, 2000).

Concepts of ability are constructed by social understandings of what constitutes competence. Cultures evolve and rely around a spectrum of ability; people's abilities are compared and judged as either unable to succeed at certain tasks ('incompetent'), to accrediting talent to learners

\footnotetext{
${ }^{1}$ In this study, I will alter the commonly used words 'Asperger's' and 'Asperger Syndrome'. To capitalise the words with apostrophes would indicate diagnostic use of disability terms within a medical discourse of ability. For instance, it is commonly reported than Hans Asperger 'discovered' the concept of 'Asperger's Syndrome' and gave this medical syndrome his name, with capitalisation and an apostrophe. Because this study is discourse analysis, I wish to avoid terminology that places my research within a medical discourse.
} 
who display successful standards of socially accepted tasks (MacFarlane, 2000). These descriptions are organised in ways that systematise and normalise situations to do with acquiring knowledge and ability (Stockholder, 1994).

Human ability is measured, categorized, compared and contrasted in most societies. As Michel Foucault argued in his writings The Birth of a Clinic (1973) and Discipline and Punish - the Birth of a Prison (1975), social systems create perceptions of what are considered normal and abnormal. Ability, effort and considered potential are constructed through social processes that create a limited number of social positions.

It is through these conditions that people develop identities, statuses and frames of thought or mind. For example, Temple Grandin was celebrated as a successful writer despite her 'high-functioning autistic' label (Grandin, 2007). However, being diagnosed autistic and 'mentally retarded' meant she experienced extraordinary obstacles of fitting in, and was stereotypically labelled as a 'geek'. As Mead stated, "Selves can only exist in definite relationships to other selves" (Mead, as cited in Bagatell, 2007, p415). The interpersonal inevitably becomes the personal. Therefore, constructing social categories is a "highly cultural and evolving process" (Bagatell, 2007, p.415). Human life revolves around social groupings that can be reliably measured within a particular society.

There remains the challenge for talented people labelled as having aspergers, of decisions of which positions are best to identify with, and whether it is possible to choose identities at all. Is a person's destined path 
determined by such labels? How can such people 'fit in' with a dualistic largely non-asperger society? Is it possible to challenge the ordinarily used, yet negative connotations, of words such as 'disability', 'impairment' and 'aspergers'?

Foucault (1975) introduced the idea of 'technologies of the self'. He argued that humans are not born as separate individuals with 'natural' status positions within societies. He described 'technologies of the self' as ways individuals act upon themselves to produce particular modes of identity and sexuality. These technologies include methods of selfcontemplation, self-disclosure and self-discipline (Synque, 2007). One technology of the self is the narrative of the autobiography.

Foucault also believed that 'technologies of the self' are part of the production of discourses that shape societies (Foucault, 1975). To Foucault, discourses describe worldviews which influence social behaviours, communications and understandings of concepts of abilities. Discursive meaning is found everywhere that humans socialize; the words people use, the behaviours, beliefs and morals people live by. Discourses shape people's social status and positioning, and hence shape the selves we can be. Discourse, therefore becomes part of 'who someone is' that it seems 'natural' to believe so (McHoul \& Grace, 1993).

Discourses act powerfully to constrain what is possible, while often being hidden from view as part of a taken-for-granted way of living. For example, society may not question normalities that are part of a medical discourse 
(Fulcher, 1989). Diagnosis is used for special educational funding, for language and descriptions of terms such as 'syndrome' or 'sufferer' or 'special needs' and scientific explanations and cures of difference to the mind (Ministry of Health, 2008). Since discourses create what is possible in society, we cannot avoid discourse, but rely on discourses, as dominant discourses and categories become imposed or 'inscribed' on people in social interaction and through institutional culture (Bagatell, 2007, p. 414).

This study focuses on the existence of many different types of discourses. Some discourses dominate others as they are practically ingrained within cultures. One dominant discourse type found in the past and prevalent today is medical discourse, as discussed by Foucault in The Birth of a Clinic (Foucault, 1973). Medical discourses have tended to place a high degree of scientific explanation, such as genetics or biology to produce certain cases (Fulcher, 1989; Parker, 1992; Wisconson Medical Society, 2007). Medical discourses have also tended to justify many social patterns, which lead to carefully considered interpretations of social standards, sanctions and scrutiny (Parker, 1992). For example, diagnosis of autism spectrum disorder or aspergers syndrome was medically developed to decide who is autistic (Autism New Zealand Inc, 2004). Societies have not only abided by standards and criterions, but have inevitably lived and been accustomed by them. Standardisation becomes normalized and unquestioned.

The paradoxical effect of people living with both gifted and asperger labels could be best described as 'juggling' the many different discourses and 
deciding whether to negotiate, adapt, defend or change attitudes and identities accordingly. Donna Williams, acclaimed writer of Nobody Nowhere described juggling the journey as 'battles to craft a self' living with in a world with high functioning autism (Williams, 1992).

One subculture this study explores is a population of people who are proudly identified as 'having asperger syndrome' (Plank, 2005). Self dubbed 'aspies', they have positive outlooks of their unique identities, despite being surrounding in perceived negated discourses in their lives ${ }^{2}$. Using a rights-based discourse, aspies tend to focus on ensuring equality and pride for those living with aspergers in a mainly non-asperger 'neurotypical' world. Yet, membership in the aspie world is not isolated to a cultural sub group, but one that exists perhaps, alongside and as a protest to the ordinarily negated term of asperger 'syndrome' (Plank, 2005). Understanding that the eccentricities, oddities and idiosyncrasies that 'make up' traits commonly shared amongst aspies are often considered by them as part of their socially inept 'geek syndrome' (Moltzen, 1995). Hence, aspies have adopted terms and words from the 'neuro-typical' normal world and characterized the usage into socially understood, positive slang meanings (Bagatell, 2007).

\footnotetext{
2 'Aspie' as a term in this study differs from the term 'aspergers'. Aspergers refers to a socially constructed and commonly understood condition or 'syndrome'; however this study views aspergers as a difference, or way of being. People with aspergers may feel proud of their difference and self identify as an aspie. However, not all people deemed to have aspergers identify themselves as aspies.
} 


\section{Aims of the study}

This study had four main purposes; first, it investigated the usefulness of the concept of dualisms, to see whether there are populations of people who describe their experiences as being classified as gifted and with aspergers as if they were on two sides of a learning ability spectrum (Bourne, 2004). Another predominant dualistic notion is 'othering'; the dualistic idea that people are either 'normal' or not (Foucault, 1975). These ideas are explored throughout the study.

Secondly the study examined the type of discourses surrounding giftedness and aspergers that participants chose to share. The study examined the descriptions of participants' emotionally colourful journeys being labelled with these terms and how their discourses were influenced and influence their social environments.

Thirdly, the study aimed to challenge the stereotypes of people categorized as 'gifted' and with 'aspergers' and/or 'disabled'. Such research could inform schools, employers, policy makers, and parents and friends who could gain an understanding of the unique experiences of being gifted and disabled (Bruzzano-Ricci, 2005).

Finally the study used a participant focus in its underlying research ethics. The study gave individuals with these identities or labels a forum in which they spoke their views of what reality of life is for them. Not only could the participants gain recognition from this study; their data may encourage and empower other people with similar identities or labels, who may 'hide' 
their giftedness, aspergers and/or disablement, to instead be proud of their uniqueness (Baker, 2007).

My study explored possibilities for appropriate methodology, given arguments from disability theorists such as O'Brien who advocated that disability research should hold her participants' views of 'nothing about us without us' (O'Brien, 2005, n.p). I worked with participants who were advisors and co-workers for the duration of the study, which enabled a richer, detailed and more critical description of their lives (Stockholder, 1994). The social and medical models and discourses of aspergers and giftedness were studied, especially the works of Fulcher (1989) and Foucault (1975), who believed disability is a social power construction from societies.

To summarize, this study adopted Foucault's theory of power and discourse in order to provide:

1. An exploration of how people classified as gifted and aspergers described their lives living with the 'dual exceptionality' labels.

2. To reveal and examine the discourses in their stories participants choose to share.

3. If there existed dualisms of gifted ability and aspergers in participants' talk and how they were used in participant's stories.

4. To explore the potential relevance of the concept of individuals classified as both gifted and aspergers in current New Zealand society. 
This study is therefore unique in that people with aspergers may be considered dually gifted and talented, although such juxtaposition may seem paradoxical to those who see these characteristics as mutually exclusive. The study also differs from traditional approaches to research about people with Asperger's Syndrome as it takes a participatory stance, portraying a shared co-researcher relationship. There are very few studies of this type (e.g. Biklen \& Kliewer, 2006) and could be useful as an alternative to traditional medical or quantitative research on aspergers and/or giftedness. Participatory research related to the gifted-asperger dualism is needed to evolve new understandings insider opinions of the positioning of people with aspergers and giftedness, and how social discourses shapes concepts of ability.

\section{Research questions}

1. If there do exist any dualisms between giftedness and aspergers, as found in the literature and expressed in the accounts of people who are labelled both as gifted labelled and aspergers, how are they expressed?

2. How do people who have been labelled as both gifted and aspergers view and describe their lives?

3. What type of discourses are used by participants and what impact do the discourses have on their lives?

\section{Researcher's background and interest to the study}

My passion for researching people who are labelled as gifted, autistic or disabled grew from my personal relationships with people who express their differences. I have two siblings who experience intellectual disability. 
My close involvement with their lives has meant a personal journey of exploring how people are labelled according to ability. Other people who experience differences to learning have also shared with me their ideas of ability; thus developing my interest of this social topic.

I have also worked in the disability field; as a facilitator and support worker in community groups for people of various ages, and as a disability advocate. These professions have included working for and with people who experience these labels. 'Asperger's Syndrome', as the idea is commonly referred to, has always interested me as people labelled as this are often fraught between what is 'normal', 'disabled' and 'gifted' and how their identities have formed according to these terms. Therefore, this study widens my understandings of what aspergers is to other people and how society can influence current knowledge.

\section{The journey of 'aspies" narratives, chapter by chapter}

In order to create a foundation for my study, I developed some definitions of the often fluid concepts of disability, impairment, autism, aspergers and giftedness, based on a critique of literature. These are reviewed in Chapter Two 'Foundations before the Journey'. Several discourses, using Foucault's framing, are outlined in this chapter, namely medical, lay and charity discourses and the traditionalist measurement discourses of giftedness and disability (Foucault, 1975).

Chapter Two also incorporated a literature review of the sub group aspie, and the creation of the term 'savant syndrome'. This provides an intriguing 
example of the interplay of ability and inability. Subchapter 'A discursive framework for exploring the asperger world' follows in Chapter Two. This describes Foucault's post constructivist philosophy of discourse analysis. 'Categories of disability discourse' is a review of some discourses disability tends to be represented by, according to other disability literature.

The uncomfortable, yet unavoidable ideas of normality and how society organizes people according to ability is explored in Chapter Three 'Veiled Genius: Manifesting Inability, Masking Talents'. This details arguments of how abilities are measured and judged, and the social groupings that result from this.

Chapter Four is a review of literature, which sought to understand the ways aspies have viewed their lives, in 'Life as We See It: Gifted Aspies' Perceptions of Life Experiences'. I reflected on the rare studies done which incorporated people labelled aspergers as narrative participants.

Chapter Five sets out my methodology, with a detailed personal account of the process of establishing a collective, participatory method. I explored literature that incorporated this fashion in a domain otherwise left unconsidered. My decision to create opportunities for participants to be co-researchers meant I sought to understand the processes that have been enabling and disabling to people deemed to have aspergers. The second part of Chapter Five details the unique quest for data, a journey 
that took me to never-revealed and unrivalled aspects of the participants' colourful lives.

Chapters Six through Nine present various aspects of the findings of my research. Chapter Six, 'Self identity As Aspie', incorporates narratives describing identity difference of neuro-typical 'normal' people, diagnosis's and a certain dignity of being aspie. Chapter Seven, 'Sharing the Aspie World', delves into the ways aspies supported one another, in order to 'survive' living in an 'uncommon world' with neuro-typical people. The language which typifies discourse is discussed in detail of Chapter Eight 'Language and Words', namely, measuring abilities, considerations of the word 'normal', stereotypes and what constitutes as 'disabled'. The final data analysis in Chapter Nine focuses on the unique views of the topic by gifted aspies themselves.

My findings developed as I addressed larger theoretical debates in Chapter Ten. Focusing on participants' personal narratives, I explored elements of aspie and gifted identity, and discourses that surround and enable these perceptions.

The concept of people with aspergers having a disability or impairment concurrently with giftedness is paradoxical. Studies done on the topic are rare; hence this research may throw a new light of 'ability' to be explored. 
$\sim 24 \sim$ 


\section{Chapter 2}

\section{Foundations before the Journey}

All humans engage in cultures which inevitably create attained ways of thinking, being and living. These cultural acts become normalised and common within cultures. Often the acts are pervasive and rather subtle, and seemingly typical ways of being are often unquestioned. For example, expressions of self-identity are influenced by cultural opinions of what constitutes social groupings of ability, talent, disability and aspergers (Synque, 2007). Societies and cultures are constantly changing; therefore personal experiences and self-identities evolve. And it is these that are loosely based on beliefs, attitudes, values and behaviours relevant to the cultures (Rapley, 2004).

This chapter outlines the definitions used within this thesis, and compares and critiques the types of definitions or descriptions of ability in other studies or arenas. I have selected a few examples that focus on the ideas of giftedness, talents, disability, intellectual impairment, autism and asperger syndrome.

Because definitions are descriptions of cultural worldviews, I have adopted discourse analysis. Discourse analysis is used in this thesis as it enables me to detach myself from my own current worldviews and ideas. Discourse analysis allows me to explore and reflect upon the discourses and worldviews or ideas used by other people, social groups and institutionalised cultures (Foucault, 1975). 
Another reason to understand discourses and definitions surrounding concepts of abilities is there is little awareness about people who seem to be labelled gifted dualistically with aspergers / disabled. In particular, New Zealand research lacks studies about giftedness and aspergers, when contrasted to other studies about giftedness concurrently with other 'deficits' or disabling 'disorder' groups (such as attention deficit hyperactivity disorder, epilepsy and dyslexia) (Blacher, 2002; BruzzanoRicci, 2005; MacFarlane, 2000). This may be due to the few studies done in New Zealand on aspergers in general, as the New Zealand diagnostic criteria were created in the mid-1980s (Autism New Zealand Inc, 2004).

Furthermore, aspergers is usually believed to be an intellectual impairment. Since it is often described with the medical term, 'syndrome', it has been associated with impaired differences in thinking, for those diagnosed or considered with this condition (Clark, 1995; McMullen, 2000). It is because of these reasons that few studies focus on the apparent 'dual exceptionalities' of people classified as both gifted and aspergers.

\section{Definitions}

In order to indicate the definitions of key terms I am using in the thesis, my own overview of key terms is given next. 
Intellectual impairment

Impairment when used in this study, describes people who are born with or obtain a deficiency, injury or difference to a bodily function, and these variances are compared to the majority who are not judged to have this variation (Bickenbach, 1994). In traditional medical terms, intellectual impairment is a distinction and difference to one or more areas in the brain, compared to most other human brains.

However my view of intellectual impairment is more influenced by social construction, which is based on the level of sufficiency or severity of the effect or outcome, as determined by the society an individual lives in (Rapley, 2004). I stress that the effect is not necessarily a deficit or gain; it is a measured difference to perceived social norms. Intellectual impairment does not imply a person has an inability to do a task using the brain. Rather, the words suggest a difference in the way the brain functions; or in other words, a variation to the majority of people in a person's given environment if the majority are not considered to have the variance.

Sometimes people with an intellectual impairment require extra support for different tasks to enable them to function within the norms of their society. If supports are of perceived quality to a person deemed to have intellectual impairment, then the impairment need not be a disturbance or hindrance for their lives (Coleman, 2001). For example, a person with aspergers who dislikes sensory disturbance of bright lights may still be able to drive or use a computer if the light is shaded or sunglasses are worn. This 
definition, therefore, values diversity and uniqueness in the way the brain works, and focuses on difference as a celebration.

Intellectual impairment, according to some social thought, is considered to be a condition or 'bodily dysfunction' that is agreed by society as deviation from social normalities when compared to other bodies (Ramcharan \& Grant, 1994; Rapley, 2004). 'Abnormality' and deviation from the 'ideal norm' are not uncommon descriptions used for generations, from medical, psychological, educational and thus social discourse (Biklen \& Kliewer, 2006).

Because the victim-blaming paradigm is prevalent in much of society today and yester-year (for instance, in psychology, education and medical thought) it has tended to belong under a label of intellectual impairment, which has negative and damaging 'tragedy' connotations as a problem intrinsic or belonging to an individual (Biklen \& Kliewer, 2006; Rapley, 2004). For example, there are accounts of parents of babies considered intellectually impaired who have often experienced disappointment or shock at the idea that their child may be a less capable human, who may be viewed by others as 'lesser' to an ideal 'perfect' child (Bennet, 2002).

Terms such as 'mental retardation', 'disorder' (i.e, not 'in order') and 'mental handicap' are words that may be considered derogatory labels (Biklen \& Kliewer, 2006; Miller, 2005). These terms and descriptions will not be used in this study, but an understanding of their cultural etymology and historical and present understanding of intellectual impairment will be. 
This study considers the history of these words and that the etymologies and associated meanings shift and evolve in common usage. It can be supposed that whilst these words were commonly used historically they may not have the same usage today. This is a reflection of historical events such as the recent closure of mental institutions in New Zealand; disability rights self advocacy movements and a growing participation of people with disabilities, impairment or aspergers in arenas that promote self worth of minority identities. Hence, words such 'mental retardation' may now be perceived as ablest and derogatory, and less politically correct. However the evolving language surrounding intellectual impairments remains a negated facet of learning abilities, and is used in manners which are not intended to be ablest, but simply to describe and compare learning ability.

Such stigmatising social forces define what is normal, and people are often positioned into categories as part of a taken-for-granted worldview that divides the normal from the ab-normal (Foucault, 1975). For instance a young child will usually be deemed 'brain damaged' before he or she can decide on a self-chosen identity to explain the socially perceived difference to their brain. People are born into social environments where, as children, they are unable to change the received social thought because the view of what is ordinary is often left unquestioned. These social influences develop into the personal opinions of people deemed to be intellectually impaired and it may be difficult to change their own attitudes and those of other people (Bagatell, 2007). 
However, over time and contexts, the dualisms of minority and majority groups always change (Baker, 2007). For example, being left-handed was once considered an intellectual impairment in western culture; lefthandedness is now not considered any differently to being right handed, despite still being a minority.

According to US government regulations, a medical view of intellectual impairment is set out as a national norm, as can be seen in the following 1997 Individuals with Disabilities Education Act regulations:

... A disorder in one or more of the basic psychological processes involved in understanding or using language, spoken or written, that may manifest itself in an imperfect ability to listen, think, speak, read, write, spell or do mathematical calculations, including conditions such as perceptual disabilities, brain injury, minimal brain dysfunction, dyslexia, and developmental aphasia.

(Cited in Blacher, 2002, p.103)

While questioning this definition, I acknowledge that people are currently and historically defined by these normative understandings. This traditional medical discourse appears to use objective, observable and intrinsic methods of viewing impairment. It also adds to a 'personal tragedy' discourse in that nothing much can be done about an 'unfortunate condition' that nature has 'given' (Fulcher, 1989). Factors such as difference of cultural values are not acknowledged in the definition; for instance, what constitutes a 'perfect' or 'imperfect' ability? The abilities listed are also limiting, hence stresses what abilities are valued in the writer's society (Blacher, 2002). 
Other literature surrounding intellectual impairment has hinted at an acceptance of diverse abilities within society, yet continues to separate 'the intellectually impaired' from people who are deemed 'normal'. For example, the New Zealand Ministry of Education use the term 'special education' to describe those learners who require specialised support to reach the measured education norms for ages (Moltzen, 1995). This is not unlike Garner's traditional approach to learning, that normality is based upon people reaching socially agreed ability level milestones at given ages (Moltzen, 1995). This theory states that should people fail to not show ability of measured social expectations, there is a problem and judged inability or lesser or lack of ability. Conversely, people who shows signs of achieving higher than the marked stages for the expected age level, are considered talented or with more ability (Cowan, Stainthorp, Kapnogianni \& Anastasiou, 2004).

However, special education in New Zealand as a legal term rarely includes those with considered advanced or higher ability talents. 'Special education' is an adopted term to portray those learners who require specialised support for not reaching ideal age related standards, often due to impairment (Bevan-Brown, 2004).

Intellectual impairment, then, is largely based upon what is considered by society's understandings, and eventual attitudes, of the concepts of abilities. These continually change and evolve, and become inscribed in social institutions. Whilst there are legal definitions of intellectual impairment in New Zealand similar to the American definitions, in this 
study I use the more reflective and social description of the New Zealand

Disability Strategy:

The common factor among people with impairments is that they face many lifelong barriers to their full participation in New Zealand society. Attitudes have been identified, through consultation, as the major barrier that operates at all levels of daily life in the general population. Attitudes and ignorance make their presence felt as stigma, prejudice and discrimination. In the year to June 1999, disability discrimination was the largest category of complaints to the Human Rights Commission

(Dalziel, cited by Office of Disability Issues, 2009, n.p).

\section{Autism spectrum and Asperger's Syndrome}

The so-called 'autism spectrum' is a label often considered an intellectual impairment; however it is a spectrum of ability in comparison to social norms (Tammet, 2006). Many people diagnosed with this label are described as experiencing some difficulties with communication, imagination and socialization as listed in the diagnostic criteria of autism (Ministry of Health, 2008). Since the forming of diagnostic criteria for autism, it has been assumed autism is not uncommon amongst all cultures, classes, ethnicities and ages (Autism New Zealand, 2004). This study takes into account of these common understandings of autism.

Aspergers is classified on the autistic spectrum. Aspergers is commonly known as 'asperger syndrome' due to the characteristic patterns of behaviour and symptoms of a disorder as mentioned above (Autism New Zealand Inc, 2004). In this study I do not use the term asperger syndrome, as 'syndrome' carries a loaded negativity about the difference of thinking that is aspergers. 'Syndrome' promotes an idea of medical pathology in psychiatry as a group of symptoms that create characteristics of a specific 
'disorder' or 'disease'. Instead, I use the term 'aspergers' to indicate a diversity of people who may use this term.

Aspergers is the main 'type' of autism grouping used in this study for two reasons. As autism definitions seem to cover a broad range of characteristics, a smaller sample of autism is more feasible for the scope of this study. Also, I am interested in aspergers, because the characteristics diagnosable to this 'syndrome' tend to be more 'hidden' as compared to characteristics belonging under the label autistic.

The socially measured characteristics of people diagnosed with aspergers are a variation of those diagnosed with autism. For example, speech is usually present and articulate compared to people with autism (Autism New Zealand, 2004). Research and current criteria for diagnosis shows that aspergers is considered a higher ability (often referred to as "higher functioning') 'end' of the autistic spectrum (Lindquist, Carlsson, Persson, Uvebrant, 2006). There are also theories that people diagnosed with aspergers may not experience the same degree of difficulty as people with autism for some tasks, such as independent living (Grandin, 2007; Tammet, 2006). Autism New Zealand defined 'asperger syndrome' as:

...a form of autism at the higher functioning ends of the autism spectrum. People with Asperger syndrome are of average (or higher) intelligence and generally have fewer problems with language, often speaking fluently, though their words can sometimes sound formal and ideas which are abstract, metaphorical or idiomatic may cause confusion and be taken literally. Unlike individuals with classic autism, who often appear withdrawn and uninterested in the world around them, many people with Asperger syndrome try hard to be sociable and do not dislike human contact. However, they still find it hard to understand nonverbal signals, including facial expressions.

(Autism New Zealand Inc, 2004, n.p., original capitalisation). 
Autism Spectrum Disorder Information Network defined 'autistic spectrum disorder' as:

...the term most preferred by parents and professionals that is often used to describe the whole range of a complex, life-long developmental disability that occurs in differing degrees of severity and a variety of forms and affects the way a person communicates and relates to people around them. This range usually includes Autism (severe, moderate, mild); High Functioning Autism; Asperger Syndrome; Pervasive Developmental Disorder - Not otherwise specified (PDD-NOS) and often includes semanticpragmatic disorders of language.

(Autism Spectrum Disability Information Network 2009, n.p. original capitalisation).

Despite the fact that these definitions direct the understandings of organisations supporting people living with aspergers, these definitions are laden with deficit connotations. As aspergers and autism are considered impairments, key words are such as 'developmental disabilities', 'degrees of severity', 'moderate, mild' 'disorder' and 'problems'. It is typical to therefore not assume talent or celebrated ability. As mentioned earlier, diversity is celebrated in this study; hence my definition of aspergers is comparatively different. In so far, differences are not necessarily considered as sufficiencies or severities, incapable nor ideal in this study. Differences are instead viewed as diversities.

In my opinion, following to some extent the social model of disability that focuses on environmental constraints that create disability more than a personal deficit (Fulcher, 1989), the 'higher and lower functioning' conception of a 'spectrum of autism', depends more on the support and environments people with autism and aspergers receive. For example, a person labelled aspergers who has an ability to be articulate may have 
barriers in his or her life if the people that socialize with the person do not allow his or her voice to be effectively heard. On the other hand, a person living with autism who cannot speak may be taught sign language in order to be given, as well as to create, opportunities to socialize with this form of communication. This study does not consider any person more able than another by impairment but more by how the society supports people with impairments to be able.

This study takes into account people who understand they are considered as autistic but feel they are very similar to the diagnostic criteria of aspergers. Because asperger syndrome diagnostic criteria were developed recently around the late 1980s, many people born before that era were considered or diagnosed as having a form of 'higher functioning' autism. My study argued people deemed high functioning may have characteristics that are a variation to the present diagnostic criteria of autism but are not necessarily more or lesser able than people without autism or aspergers, because this depends on the type of support available for their needs and wants.

Authors Biklen and Kliewer (2006) argued that words associated with impairment such as 'mental retardation' are neither natural nor 'real' but socially perceived. Nobody is born with autism or aspergers, but the behaviours and characterises are labelled according to those using power to categorise, such as people in medical professions. For instance, these conditions are not owned by people so labelled as autistic. As the authors argued against medical descriptions and diagnostic criteria, they asserted 
"autism, mental retardation and competence are what any of us makes them" (Biklen \& Kliewer, 2006, p.183).

Rapley (2004) agreed that autism and other related intellectual conditions are usually considered by society as an individual problem; in fact it is society that creates and shapes impairments and disabilities (Rapley, 2004). For example, the definition used by Autism Spectrum Disorder Information Network stated autism spectrum disorder is "a term most preferred by parents and professionals" (2009. n.p). My study stresses that disability professionals, educationalists, psychologists and other medical professionals created the ability metaphors that became real to the rest of society. Such professionals who defined the beliefs, definitions, limitations and labels were usually those not categorized in the ways they purported.

Biklen and Kliewer (2006) advocated that the formal definitions of 'mental retardation' are crudely inadequate and debilitating. They stated the concept is grossly unfair for any person to be so labelled". Definitions such as those given by the disability writer Blacher (2002) were considered authoritative, taken from traditional medical discourse of intellectual impairment and disability. Words and language are instrumental to social norms, thought and values.

Blatt and Kaplan (cited in Biklen \& Kliewer, 2006) added that 'mental retardation' is but a metaphor, and the term is terribly dangerous and disenfranchising. The authors pointed out that people generally have not 
met any human who chooses to be called mentally retarded, although people exist who are quite comfortable to place that appellation onto others.

Despite the prejudice and negativity of these worldviews, it is important for this study that I understand and reflect these historical and yet still current paradigms of thought that remain alive in modern society. The terms 'autism', 'aspergers' 'intellectual impairment' and 'disability' have been used intermittently in literature (Plomin \& Price, 2003). Other more historical words used are 'mental retardation', 'mental' or 'intellectual handicap', 'lame', or 'dumb'; however these words can still be found in academic writings or heard in social utterances (Stockholder, 1994). From reflecting past attitudes, behaviours, beliefs and discursive expressions, can I seek to understand how people who are deemed as autistic or aspergers describe their identities and lives.

\section{Personal identity of 'aspie'}

There is a current trend for people labelled aspergers to reflect on and choose to have a positive insight and self identity of aspergers (Bagatell, 2007). Self dubbed 'aspies', their life stories often convey the hardship of self acceptance of having a difference; however this self acceptance is often made challenging as people without the label of aspergers struggle to learn and accept their differences.

Similarly to the evolving language of the words 'mentally retarded', or 'handicapped', aspies exist as a social group that is made real when used 
commonly amongst a culture with shared understandings and characteristics of the group (Bagatell, 2007). In contrast to negated words however, 'aspie' can be seen as a positive term used by and for people who confidently identify as having aspergers. It also differs in that aspies created their name, of their opinion and 'type' of autism themselves, rather than being forced to be positioned with a label from others. This gives aspies group support to enable possibilities of becoming proud together as a sub-culture (Plank, 2005).

Some aspies have used creative words amongst themselves. From personal experience I have heard common slang within this group include phrases such as, 'neuro-typical' to describe people not deemed to have aspergers. Another example is when aspies have described a person in the form "he/she is an aspie", as if the word 'aspie' is a noun; and "I'm aspie" as if describing an identity or a personality trait (Bagatell, 2007).

'Aspie' therefore could be seen as adopting and challenging a label, and perhaps changing its negative force to a more positive one ${ }^{3}$. Because of the nature of participatory research, the word 'aspie' is adopted as a result of participants' choice in describing their identities.

Interplay between Asperger's and 'savant syndrome'

'Savant syndrome' is a term that has been used by other authors to describe learners with intellectual impairment who need not be taught a

\footnotetext{
${ }^{3}$ This is similar to gay or lesbian people adopting on the word 'queer' as an identity which had previously been pejorative
} 
skill, or need very little practice, to have considered a sudden amazing gifted ability in a short amount of time. Treffert defined savant syndrome as:

A rare, but spectacular, condition in which persons with various developmental disorders, including autistic disorder, have astonishing islands of ability, brilliance or talent that stand in stark, markedly incongruous contrast to overall limitations

(Treffert, 2007, n.p).

Placing this condition as a 'disorder' and 'syndrome' has developed a worldview based around medical assumptions: that people with intellectual impairment and giftedness are lucky and fortunate to have giftedness to 'save' them from a predestined life of impairment, disability, or 'dis-order' of normal development. Because the talent is considered as often unexplainable, it appeared, by Treffert's definition, a mysterious innate or fixed trait of people called 'savants'.

Some writers who have been considered 'savants' or with and 'savant-like' skills are due to a genetic basis (Cash, 1999; Clark, 1995; Grandin, 2007; Treffert, 2007). This is seen in Grandin's belief that:

The disorder may occur if a person receives too big a dose of genetic traits which are only beneficial in smaller amounts. For example, a slight tendency to fixate on a single subject can enable a person to focus and accomplish a great deal, whereas a stronger tendency to fixate prevents normal social interaction...

(Grandin, 2007, n.p).

In this statement, the savant condition or syndrome is considered a special talent. The medical label already shows that it can be measured and tested to see who fits into the stereotypical norms. Cash also added: 
The genetic traits that can cause severe disabilities can also provide the giftedness and genius that has produced some of the world's greatest art and scientific discoveries...

(Cash, 1999, p.23)

From this statement, genetics plays not only a part of defining who has savant syndrome, but that it can be viewed positively as a useful condition for society.

My thesis will also question the traditional views of the savant syndrome, as this syndrome sits within a medical discourse of impairment being intrinsic and unusual within the individual. Literature seems to suggest that simply because some people with intellectual impairments have talent comparable to most people with impairments, that these people must be somewhat rare, mysterious, and hard to comprehend, and be given a mysterious French label. The medical discourse tends to portray people 'with intellectual impairment' as very rarely, achieving talent that matches those without intellectual impairment (Morelock \& Feldman, 2003).

I will explore ways that this discourse continues the dualism of a disabled minority and non-disabled majority and creates the unfair stereotypes mentioned. If this is the case, gifted people labelled aspergers may be poorly supported in New Zealand education, employment and social life (Bourne, 2004). This research has been influenced by the New Zealand Disability Strategy, which stated:

Many impairment groups have rejected being called 'disabled' at some point, usually because of negative connotations associated with 'disability'. They have argued that 'there is nothing wrong with us; we are just different, with different support needs'.

(Office of Disability Issues, 2009, n.p) 


\section{Disability}

'Disability' as a term has various and arguable definitions, mainly because disability is found in all societies, classes and cultures; it is because of these social paradigms a category, or categories of disability are enabled. For example, the term disability is interpreted variously with regard to its usage in literature and speech, from medical, psychological, social, or media discourses (Fulcher, 1989). Often the term is used intermittedly with terms of intellectual impairment (Biklen \& Kliewer, 2006). Many authors tend to share the term rather loosely; to portray a dualistic nature of 'ability' and 'disability' (i.e. disabled being the opposite of abled) (Rioux, 1994). Such categorizing, therefore, creates a description for people believed to be unable to do or be something as compared to people deemed non-disabled.

This thesis takes as its starting point the social model of disability (Oliver, 1997) and has morphing infusions of Michel Foucault's discourse of disability (Horrocks \& Jevtic, 1998). In this study, disability is a term used for people who have experiences of disablement due to circumstances in their life that prevent them from doing a task or taking part in an activity, compared to people not limited due to barriers. The term disabled does not imply people are impaired because they are born with characteristics seen in the diagnostic criteria of aspergers. There remains a vital difference between 'impairment' and 'disability'. Also, social forces can influence a person who has a considered impairment to have or not have the identity or label of disabled. For example, a person with aspergers may 
have a visual impairment but because they wear glasses are not called disabled.

This study does not exclude the stories told by those people identified as with aspergers but who feel they are not victims of ablest attitudes or have challenges of disablement. To limit this study to people deemed as having aspergers and whom have experienced ablesm assumes that aspergers is a disability.

Fulcher (1989) argued that disability is a procedural category, rather than as something people cannot do; as a category it is a term used in discursive practices where impairment may or may not be present, but where the presumption is made that it is due to barriers. She added that presumptions about the presence of impairment should be suspended because disability is relative to social practices and independent of the presence of impairment. For instance, the 'same' impairment does not always lead to an identity or labelling of disability. On the other hand, if someone is born with features that are considered impairment, such as those used to determine or describe aspergers, an assumption is they are also intellectually disabled.

It appears that impairment and disability go hand-in-hand in much of society. As Foucault and others state (Biklen and Kliewer, 2006; Rioux, 1994; Synque, 2007) a person is not disabled due to their impairment; they are disabled due to barriers put in place by society. People with autism may not be disabled if they receive the right support in their lives. 


\section{Giftedness}

This study focuses on celebrating talented achievements of people categorised as aspergers. I do not detail which gifts or talents these may be, what measurement structure I believe is best, or ways used to establish ability level. However the talents are skills that are perceived by either the participant or society as an ability that is considered to be at a socially high standard compared to norms.

Talents therefore are undeniably subjective and questionable; this is because society often groups ability to certain standards and worthiness, and these standards constantly change. This is not unlike how art may be interpreted and are subjectively and ongoing judged; some people may view certain 'works of art' as unique creations, or not artistic at all. Therefore, given diverse standards of perceived ability, giftedness may be what participants or others 'make of them'.

Traditionally, intelligence has been the measurement of giftedness (Biklen \& Kliewer, 2006). Intelligence and giftedness is also measured on effectiveness, worthiness and productiveness to society (Gallaher, 2003). Historically and today, many writers used results from intelligence quotient tests (e.g. Baum, Herbert \& Renzulli, 1994; Bourne, 2004). Relying on these scientific and objective methods limits the relative potential found in individuals categorised as having disabilities and aspergers, which may express giftedness in ways that are not typically found on written and reading tests. 
George Parkyn, founder of New Zealand gifted educational schools, described the details of the giftedness definition used for the organisation he began:

When we turn to the problem of identifying the gifted and encouraging the development of their giftedness, two basic aspects need to be considered. The first is the ability of gifted children to progress rapidly through the known and accepted stages of development of rational thinking, ethical sensitivity and aesthetic awareness. The second is their potential ability to achieve new insights beyond those already known.

(Parkyn, cited in the New Zealand Association of Gifted Children, 2009, n.p).

Parkyn's words 'known and accepted stages of development' suggests the intrinsic traditional model of giftedness, as reflected by institutionalised society. This is further enhanced by the New Zealand Association of Gifted Children website's (2009) descriptions of how giftedness was decided upon, in order to become a parent member:

Eligibility for joining a branch of the New Zealand Association of Gifted Children.

Guidelines:

Some formalised testing that indicates the child is working or able to work at or above the 95th percentile; e.g. school reports or tests, extra-curricular activities, etc.

Specific information about the child's learning, social behaviour or development indicating ability in the gifted category, e.g. reading well at three years of age. Examples of the child's work, e.g. writing, art, maths.

The ability to competently handle curricula standards set for an age two years or more above the child's age.

(New Zealand Association of Gifted Children, 2009, n.p.).

Binet coined the concept of the 'mental age': children grow in intelligence and that any given child may be measured ahead or behind the typical 
level of his or her actual age (1907, cited in Reis \& Ruban, 2004, p.155).). Whilst this concept may be applicable in most contexts, Binet's comparisons gave no consideration to different class or status, ethnic cultures or generations. His related notion is that children who learn the most do so because of 'greater intelligence'. This idea grounds the medical, traditional discourse of giftedness and disability. Binet did have a point however; simply as Gallaher's (2003) writings about giftedness being reared as part of socialisation: "Intelligence is malleable and capable of improvement if we pay appropriate attention to it". That is, social factors influence the outcome of measured ability status (Binet, 1907, cited in Reis \& Ruban, 2004, p.155).

It is important to note, however, that many abilities that may be considered 'gifts' are not easily measured, or may not be related to traditional notions about the intelligence quotient (IQ). For example, the concept of creativity is by definition subjective and fluid, and differs according to cultural ideas about what constitutes art, imagination and originality. Gardner's theory of Multiple Intelligences explains that talent may fall within eight possible intelligence areas, and range from bodily / kinaesthetic to visual/spatial intelligences or more (Taylor, 2009). So whilst IQ tests may be useful for measuring specific abilities for specific reasons (e.g., to work with students with particular gifts in vocabulary or spatial skills), the tests cannot be generalised for all gifts and talents. They exist only as a type or test useful in some circumstances. 
Theories and concepts of giftedness have changed and evolved historically. The recent decade has shown recognition of gifted individuals within diverse social groups, such as those with asperger syndrome. Whilst medical studies have tended to be grounded in traditional empirical notions of IQ (e.g. Clark, 2995, Rioux, 1994) there has been a growing shift in research that has acknowledged that people with aspergers are talented. The change in discourse has meant for some researchers a difficulty in determining which traits of gifted people with aspergers are used to define aspergers, their perceived giftedness or if the characteristics are unique qualities not explained due to giftedness or aspergers (Cash, 1999).

Cash (1999) argued that individuals with aspergers and gifts are more easily tolerated by society as they are able to make their own accommodations in everyday life. She suggested that "the key to their success and growth appears to be their higher ability to manipulate and thereby dilute some of their apparent autistic weakness and tendencies" (Cash, 1999, p.8). Despite her recognition that people with aspergers may be talented, she described aspergers as intrinsic to the body and a reason for impairment or disability. Any apparent gifts are also viewed as intrinsic and deemed fortunate to lessen the disability, therefore developing a dualism of higher and lower ability.

More recently, other writers have stated that schools, employers and other key holders who are involved in 'gate keeping' the perception of competencies through processes such as assessment, should be 
accommodating by allowing more opportunities for people with aspergers to show their unique strengths. This stance takes the approach of a celebration of pride of diversity, especially minority groups such as those with aspergers. This can be seen in the research of Clasen and Clasen (1997). They argued that mentoring individuals to present their abilities in a good light may triumph over disability or impairment alone: "The mentorship may enhance the young person's sense of self-worth and an appreciation of the potential to be tapped". Mentorships are therefore ideal for people with giftedness and aspergers, as confidence in their identities as unique human beings can develop without lessoning individuals' realities of difference, whether perceived impairment or not.

New Zealand established a Working Party of Gifted Education in 2001 to develop goals to sustain and develop potential giftedness in schools, providing, for example, professional development for educational professionals of all kinds, information technology initiatives and a giftedness advisory committee (Ministry of Education, 2002). These policies are consistent with the concept that giftedness may be found in every social group. This also reflects a change of thinking compared to earlier decades of educational policies whereby giftedness was rarely acknowledged in asperger or disability communities, or received as much attention or funding as special education in New Zealand.

Traditionally, western societies have a long-standing contradictory lovehate relationship with giftedness and talent (Colangelo \& Davis, 2003). Society admires talented individuals who are driven and eager to learn. 
These learners are often revered as those that 'give more back to society' than people labelled as disabled and assumed unemployable (as opposed to unemployed). Robert J. Sternberg, American writer on gifted education, once wrote,

Giftedness is arguably the most precious natural resource a civilization can have. Children who produce and create well beyond our expectations invigorate us and show us the possibilities of human potential. The authors have never met teachers more excited about teaching than those who work with groups of gifted children.

(Sternburg, 1986 cited in Colangelo \& Davis, 2003, p.5).

On the other hand, societies often have an educational and welfare commitment to egalitarianism, that 'all people are created equal' (Colangelo \& Davis 2003). This can be seen in the more present educational policies in New Zealand, such as the Special Education Policy Guidelines. In this way, all individuals have the right to a fair and reasonable education. 'Reasonable' education is subjective as there may be a challenge of using special educational funding for those students who need more support to reach the ideal 'normal' standards, such as a child labelled as autistic. However does this differ to another child who needs extra support because his level of ability far exceeds normal expectations? Does it change if a child with exceptional talented 'higher' ability is considered as having aspergers? The idea of egalitarianism creates the confusing tension between encouraging yet restraining individual accomplishment.

Furthermore, some writers such as Colangelo and Davis (2003) stated that disability as a category is used as a political grouping to establish which funds are to be used for certain individuals. This is often seen as 
equity in New Zealand schools (Bevan-Brown \& Carroll-Lind, 2008; Riley, Bevan-Brown, Bicknell, Carroll-Lind \& Kearney, 2004). However, students who are labelled as gifted (including students with intellectual impairment) may miss out if their giftedness is not obvious or realized by teachers, educational staff or parents. In fact, giftedness may be masked or not considered if their unusual behaviour, inability or diverse communication or apparent intellectual impairment is more obvious (Biklen \& Kliewer, 2006; Blacher, 2002; Bourne, 2004; Braggett, 1998). Furthermore, treating equity and excellence as mutually exclusive may be destructive to development of appropriate educational practices (Colangelo \& Davis, 2003).

Colangelo and Davis stressed that society may also hold a fear that one person or group may be perceived as inherently superior or hold more value than others; this feeling may also exist amongst people considered aspergers, gifted or both (Colangelo, 2003). For example, a student who is classified as a 'mathematical genius' may be viewed as more valuable to society than a student who is classified as aspergers. One the other hand, a person classified as both aspergers and mathematically gifted may be viewed as more valuable than as someone known as having aspergers with no mathematical giftedness. This is an example of a traditional discourse prevailing social thought, and adds to Foucault's (1975) debate that power is indeed an influential, social bind.

Furthermore, some people may feel hostile and may express anti-elitism to a person or group perceived as intellectually gifted (Colangelo \& Davis, 
2003). For example, if the person considered gifted in the above example was talented in sports, they may be socially appreciated by peers, teachers and society more so than if they were gifted in mathematics or writing, or other skill.

Therefore, giftedness, especially giftedness seen in people classified as aspergers, is a particularly charged issue (Colangelo \& Davis, 2003). Unfortunately, most people with labels of giftedness and aspergers are likely to have limited support within education, employment of social life (Cash, 1999). This is often because differential 'treatment' for high achievers may be of low national priority, particularly for people labelled aspergers (at school, work, family life etc). Therefore, gifted people without suitable support could suffer psychological damage and permanent 'impairment' of their talents and abilities (Gallaher, 2003).

\section{A discursive framework for exploring the 'asperger' world}

What is discourse?

Theoretical ideas about discourse come from a philosophy that constitutes the foundation for people to attain certain ways of thinking, behaving and being. That is, an examination of discourses is one way of explaining the complexities of how humans socialise and structure society (Foucault, 1975). Discourses are made up of culturally and commonly shared ideas, concepts, understandings and logics. These qualities bring about systems, orders and ways of organising people and things (Burman, 1991). In other words, discourses are socially shared ways of looking and describing the world. 
Discourse in this sense differs from the traditional 'discourse' readily used in linguistic and psychological schools which describe communicative narratives. Discourse theory in this study is comparative in that it subjectively describes the intrinsic social choices people make and do. However it is contrasting to traditional schools of discourse in that it is so much more than words and communication (Parker, 1992).

Foucault would argue that discourse is to do with objects and subjects (Horrocks \& Jevtic, 1997). To reference something, to create a name for a noun, thing, or way of being, comes to give an object a reality (Parker, 2003). The development of phenomena by discourse can be best described as 'representations' of the world which Parker contends "have a reality almost as coercive as gravity, and, like gravity, we know of the objects through their effects" (Parker, 1992, p.6).

Discourse is a whole coherent system of socialisation, and compliments the ideas known in a society. When something becomes 'known' it becomes 'real' to people in that culture, and a part of specific discourse (Parker, 1992). For example, autism has been considered a 'type' of intellectual impairment and people described as autistic share similar characteristics and perceived impairments (Autism New Zealand Inc, 2004). The usual social positioning of people with autism is focussed on perceived limitations of disability. Over time, aspergers became a syndrome related to autism. 
Discourse in a sense, creates or 'brings' phenomena into human reality. Discourse shapes the social world. Parker concisely sums it as "discourses allow us to see things that are not 'really' there, and that once an object has been elaborated in a discourse it is difficult not to refer to it as if it is real" (Parker, 1992, p.6).

Discourse do not simply describe ways of being, they also categorise groups of people (Parker, 1992). For example, before aspergers was 'discovered' with diagnostic criteria, people with aspergers were often stereotypically deemed as 'unusual' or 'eccentric' individuals (Grandin, 2007). Some people with aspergers reported feeling distinctly different to people who are not classified as having aspergers, and some consider themselves as 'having something wrong with them' or diagnosed as readily known and discovered 'mental illness' to explain difference (Grandin, 2007; Williams, 1992).

Parker believed that discourses existed as referring to things that were always there to be discovered (Parker, 1992, p.5). Discourses enable frameworks for challenging the value of one way of thinking and talking about reality over other ways. Opinions are constantly changing about aspergers, for example, discourses surrounding the idea of aspies being a sub-group membership identity (Plank, 2005). That is, discourses create a particular reality, and in this sense, transform the world. 
How is discourse used by people?

Because people's social understandings, ideas about life and worldviews constantly change, discourse is developed, used and influenced - by all humans (Ramcharan \& Grant, 1994). People live discursively, that is, the things people do depends on their discourses. For example, parents rely on diagnoses for autism or aspergers because the cultures they live in understand that doctors, psychologists and clinicians are experts of what constitutes autism and aspergers (Bruzzano-Ricci, 2005). Humans for generations have trusted medical professions as they are known to heal, find answers and 'treat' deviance to what is viewed as 'abnormal' (Bickenbach, 1994). Discursive behaviour, communication and ways of thinking happen almost without realisation - in this sense, 'intrinsically'. This is because life is socially normalised that often people do not stop to challenge a set way of being (Bagatell, 2007).

Fulcher (1989) described discourses as socially shared realms that "articulate the world in certain ways; they identify problems, perspectives on those problems and thus 'solutions"' (Fulcher, 1989, p.8). Discourse contains social rules about how aspects of how the world works and 'ought' to work for humans. Therefore all discourse is subjected to scrutiny for which worldviews constitutes a preferred ways of living. The best considered option for deviance to social norms depends on which society, culture, generation and community values are prevalent at the time that people may follow the normal 'matter of course'. Historically parents were notified to place their children in psychiatric institutions by doctors, as intellectual impairment was discursively feared. Institutionalisation was 
therefore considered 'for the best interest for the child and society' (Foucault, 1975). Parents may have been faced with ablest resistance had they decided to challenge the medical system to not send their child to an institution and instead attend a 'regular school'. Therefore, discourse is a force to be reckoned with, to rely on, change or manipulate (Fulcher, 1989).

\section{Who is Michel Foucault?}

This thesis uses the view of discourse most closely associated with Michel Foucault ${ }^{4}$. Foucault was one of the first philosophers to describe in complex detail 'socialized subjects' (Biklen \& Kliewer, 2006; O'Farell, 2004; Ramcharan \& Grant, 1994; Stockholder, 1994). According to Foucault, humans have historically been seen as the subjective object in generations of historical, philosophical, scientific, economic and legal and social practices (Foucault, 1975). Language, communication, codes of behaviour and self-identities, are thus shaped and designed by the forces of a particular social body. For example, if a medical discourse, educational discourse and political discourse, appear to position people with mathematical giftedness as 'genetically superior' compared to people considered 'non- mathematically gifted' then the people within this realm of understanding would have some influence of their lives. Funding may be used in ways to promote such talents; teachers, parents and peers of people considered gifted may have an understanding that such people are fortunate and words associated with this idea may be 'lucky' or 'natural

\footnotetext{
${ }^{4}$ Foucault was a French historian, philosopher, critic and sociologist. He has been described as 'post modernist' however; his need to critic labels and social groupings meant his rejection of an identity or association to this concept (Horrocks \& Jevtic,1997). 
talent'; such people may find they receive respect, admiration of their ability or envy from others.

Foucault argued that science classifies the individual as subject of life (biology), labour (economy) and linguistics (language) (1973, cited in Horrocks \& Jevtic, 1997, p.6). Humans are forced into subjects of social groups, within the above categories. For example, a person with mathematical talent may be labelled gifted due to genetics (biology), education (economy), historical accreditations of talented people, knowledge and discourse of what constitutes as normal and 'abnormal' ability in that person's society (linguistics). In other words, discourse does not simply describe beings into subjective objects; it also positions them within a holistic realm of being, in various social domains (Foucault, 1975).

Foucault argued that people's selves are a product of the society and context in which each person interacts (Baker, 2007). He rejected the idea that people are predisposed or have a 'fixed' essence of certain abilities or traits, influenced instead by their milieu or language and culture (Parker, 1992). A person labelled as aspergers may be influenced to decide to identify as disabled, or aspergers, or feel they are born impaired; therefore, society influences this decision hugely (Rapley, 2004) ${ }^{5}$.

\footnotetext{
${ }^{5}$ This differs somewhat to the social model of disability, which states society creates barriers to participation.
} 
Why is discourse used in my research about 'gifted aspies'?

My aim and purpose of the study is to discover the type of discourses used within the narratives of people who are described as having both aspergers and gifts, as a subjective and reflective approach. A discourse approach is the best method to benefit this study by describing the participants' worlds and examining these stories in a wide discursive lens.

Discourses also serve to explain the power relations within institutionalised societies. All social groups have a social status, and thus discursive analysis enables a critical look at how gifted people and aspies become so labelled, as well as those associated with both categories. Discourses also help to explain why giftedness and impairments or disabilities are viewed as dualisms rather than the spectrum used to describe the syndrome of aspergers on an autistic spectrum (MacFarlane, 2000).

In respect to the particular topics of aspergers, aspie, giftedness, talent, ability, impairment and disability, these words, by usual discursive definitions, should not exist in the same discursive position, or within the same person (Lyons \& Fitzgerald, 2005). An analysis of discourses breaks down the traits (labels) and subjectively explores how and why the traits and labels are placed upon a person, and the meanings behind such words.

\section{Power and discourse}

Foucault argued power is a social construction that is exercised, rather than an innate or natural possession (Ramcharan \& Grant, 1994). Learned 
power statuses are created by all humans engaging in social structures, which cause such dualisms as disabled and the non-disabled (Rapley, 2004; Stockholder, 1994). For example, educators, funding agencies and parents may view a boy who has autism as disabled, rather than capable and talented, compared to the majority of non-autistic children. The fact his label is firstly autistic creates a way of thinking about his abilities. With each person participating in the same general belief of these institutions, the likelihood is that the boy may feel outnumbered if he tried to use some power himself to prove he is capable and talented first, then disabled, if he feels he is disabled at all.

Having fewer rights, does not mean having less power; rather it shows a use of power by conforming to those that choose to execute power as force, authority or control. People cannot exist as selves alone; they exist as inevitable socialised subjects with identities and power relations based upon experiences with cultures. This corresponds with Foucault's (1975) belief that scientific knowledge is linked to power, rather than truth (truth being a subjective element).

For Foucault, power is not so much viewed in terms of one's domination or assertiveness over another, who appears to have or own power, but that all humans are part of the mechanism or structure of power in society (McHoul \& Grace, 2000). As he stated, social structures

...Become the vehicle of that power, which in turn, has constituted it as that type of vehicle. Power is bother reflexive, then, and impersonal. It acts in a relatively autonomous way, and produces subjects just as much as, or even more than, subjects reproduce it.

(Foucault, 1977, McHoul \& Grace 2000, p.22). 
Therefore, one cannot demand that a society 'gives' or 'restores' the rights of an individual or group. This is because humans are all working within the movement and flow of power; humans are the product of power (Foucault, 1975).

This view is somewhat different to those held by writers who believe there is an apparent 'top-down' power hierarchy whereby disabled rights is often the social discourse put forward (Biklen \& Kliewer, 2006; Goggin \& Newell, 2005). Often this discourse was created by an historical influence and consequence of ablesm to people who are considered disabled. However, by believing that certain people, groups or institutions have power or have more power and that people labelled as disabled have less rights and voices heard, adds to the commonly thought idea power is unevenly distributed. As Foucault stated,

Do not demand of politics that it restore the 'rights' of the individual, as philosophy has defined them. The individual is the product of power. What is needed is to 'de-individualize' by means of multiplication and displacement, diverse combinations. Do not become enamoured of power

(Cited by Horrocks \& Jevtic, 1997, p.120)

Foucault (1975) gave the concept of partnering power and knowledge as if the two terms entail one another. In a sense, they do, as often discourses reproduce power relations such as particular medical discourses of disability, whereby a disabled person may be described as 'mad' and viewed as 'unable to reason' (as written by Foucault's description of 'madness' during the time of Age of Reason, 1977) (Encyclopaedia Britannica, 2009). Therefore, forced treatment of people with madness 
was punishable during this discourse in order to 'make' a person 'reasonable' (Foucault, 1977).

Parker (1992) warned that power and discourse are not the same thing, and should not be confused with one another. Power may be coercive or resistant, and usually has subjects whom are dominating or inferior. Power may often be used as the concept of the giving and taking away of rights, rather than giving and taking away power. Discourses often challenge power, and may at times be confronted with oppressive discourses (Parker, 1992).

\section{Language and communication and discourse}

Policy researcher Fulcher (1989) wrote that language is the instrument of power in disability discourse. But this depended on which discourse is used, as they deploy various themes, styles of statements and inherently different objectives. In other words, discourses create discursive practices, and language is one instrument of practice. For example, the idea of 'integration for disabled students' can be argued by some that it has always been present in schools (for example some students are mainstreamed in regular classes) whilst other people may think the words 'integration for students with disabilities' mean full participation to every area of educational life such as aggregated classrooms and activities such as school bands, camps, outings and plays (Fulcher, 1989).

Fulcher (1989) further argued that policies and legislations are where institutional conditions are established. These discursively provide the 
bases for the words used in societies. So, if mainstreaming were decided by government to mean every child with a disability is integrated in all areas of school, educational institutions, families and society would follow suit as this would be the chosen norm, and in a sense, the chosen language of the time. As Parker wrote, "texts are delimited tissues of meaning, reproduced in any form that can be given an interpretative gloss" (Parker, 1992, p.6).

So, if disability is a category created by language, it occupies the same place as other categories, which are part of society and essential to human life. For example, 'prodigious', 'giftedness', 'talent' versus 'disability' tend to imply positive or negative (as binary opposite) meanings. However as Fulcher (1989) mentioned, too often categories such as disability is used to exclude rather than include, oppress rather than enable. Language often clouds society, words, images and behaviours exhibited of people stereotyped as a category, that the assumption of intelligence could be unfair. Following the work of Biklen and Kliewer (2006), if society were to view the world from the eyes of the child, then one may safely assume the child is far less disabled than first decided upon by others.

\section{Categories of disability discourse}

In order to describe the discourse analysis of this study, I must first outline the considered established discourses of ability, namely, disability. This is because disability is a social and political category and other categories 
depend on measurement and comparisons of it. There are many forms of disability discourse as outlined by Fulcher (1989).

Medical discourse portrays disability as intrinsic to the body, as part of a person's problem and sometimes viewed as needing to be 'fixed' medically ${ }^{6}$. Institutionalisations by way of mental hospitals were often found in medical discourse. This has a focus on 'treating deviant patients' whose disabilities are seen as abnormal - conversely treating a society as it separates the dualistic groups and shuns disablement from the community. Fulcher (1989) believed disability is feared or seen as less than perfect; despite the contradictory nature that all people may be disabled in any given culture as disability is a social spectrum. Institutional hospitals may no longer exist in New Zealand but that is no assurance that medical discourse is not alive. For example, New Zealand Special Education funding is used to measure disability and impairment as according to diagnostic tests, learning assessments and medical reports. Disability in a medical discourse continues to focus on medical knowledge and may be viewed with a negative deficit focus (Morelock \& Feldman, 2003).

Charity discourse defines people labelled as disabled as in 'need of help', such as objects of pity, similarly to medical discourse that their challenges are personally tragic. Disabled people may be considered 'eternal

\footnotetext{
6 Medical discourse is not unlike that stated in the 'Definitions' section of 'Disability' (earlier section of Chapter Two 'Foundations before the Journey'). This type of discourse tends to add weight to much of other disability worldviews and discourses in history and today.
} 
children', lower achievers by ideal standards at school, employment and society in general (Fulcher, 1989). Such beliefs carry a tone of sympathy and theme of benevolence, in which the people labelled as disabled are in need of care or protection for the rest of their lives. Examples are current sheltered workshops for people deemed unemployable or uneducable, without pay. Such discourses hold the belief that it expects its 'clients' to be grateful recipients when non-disabled people support them to accommodate into a predominantly non-disabled community. This discourse deflects attention from individuals' perception and wants; it may anger or humiliate those people who want to be taken seriously and be independent in their lives (Williams, 1992).

Another disability discourse Fulcher (1989) identified is the lay discourse. This incorporates issues of control over the human body labelled as disabled. The abuse of forcing lobotomies onto people with intellectual impairments is an example of professionals controlling other bodies. The common belief was the controlling was seen as necessary, 'for their own good' (Fulcher, 1989). In this discourse, people deemed disabled experience such controlling as ordinary and expected in their lives not realising they may be also be able to choose not to be controlled. "Less easily regulated bodies are denied the full personal autonomy expected of those whose bodies are more easily controlled" (Williams, 1992, p.30). For instance, contraceptive injections taken to avoid a birth but with little understanding or choosing by women deemed disabled. This is informed by the medical discourse, and has an ethos of charity ethic and fear, prejudice, pity, ignorance, misplaced patronage and even resentment toward people disability (Fulcher, 1989). Abberley said: 
For disabled people the body is the site of oppression, both in form and in what is done to it ... the modern preoccupation with the body is as an image systematically devalues those whose disability is based in a visible (physical) impairment.

(Abberley, cited in Fulcher, 1989, p.30).

The superhero discourse exists when people are viewed as able to do a task despite disability or impairment. The task done by someone with impairment may be judged as ordinary or common or perhaps extraordinary to a society, but not usually viewed as easy for someone with perceived impairment (personal communication, IHC, 2008). Whilst the discourse attempts to portray a 'celebration' or accommodation of difference, it also perpetuates the labelling that impairment deviant to normality hence creates a disablement. In some cultures, impairment is seen as 'closer to God' or more 'special', as if the label emphasises a goodness of impairment yet acknowledges perceived hardships at the same time (personal communication, Nisha, 2008). Therefore the discourse treats disability as a separate, dualistic nature, in which giftedness is rare.

There also exists the rights discourse (Fulcher, 1989). The main themes surrounding this are the need for people deemed disabled to have selfreliance, independence, and as consumers, deciding on wants (rather than only needs), equality of citizenship, and creating strategies of confrontation, assertiveness and demand (Plank, 2005). Within a humanitarian paradigm, the rights discourse argues that for too long people deemed disabled have been positioned as 'victims' of forced discrimination, social exclusion and oppression. 'Aspie' as a chosen 
identity is another example of creating a change to the way aspergers is viewed by aspies and neuro-typical people alike. This discourse has an overtly political feel, and a dualistic belief that it is the non-disabled people who hold all the power.

The rights discourse differs somewhat to the discourse Foucault (1975) advocated. Rather than the power being owned by certain groups of society (e.g. doctors being more powerful than other vulnerable groups) power is instead an energy that simply exists in society. Humans use the power in different ways toward each other so that norms, categories, beliefs and assumptions are created from power. Language, behaviours and communications are instruments to social power. Therefore, 'empowerment' in its current usage to 'give back power' is void in Foucault's concept of power (Foucault, 1975). How power is used depends on the forces from groups of people onto others, who may choose to argue, advocate, rebel, comply, accept or assume a normal use of power.

A small and evolving discourse that perhaps sits unsurely on the 'disability' arena is suggested by a group of predominantly 'out' and proud aspies. A growing trend for people with aspergers is to view their differences not as a disability or impairment, but simply as a difference. From recent studies (e.g., Bagatell, 2007) and my work in the aspie community, I think there may be a newer, and perhaps younger, 'disability' discourse although the difference between 'being different' and 'impairment / disability' may be blurred. The growing recognition of this discourse may be less known to 
so-called 'normal' society, as aspies in recent years have tended to find one another through technological advances such as websites, blogs, forums and social networks. Some aspies have children who are also labelled as having aspergers, which may explain the high number of teenagers and young adults who strongly identify with the term aspie. This discourse has a less political and activist stance than the rights discourse, with more effort put into changing the meanings of labels that define a disabled type. This emerging discourse may not seek to 'alleviate' aspies' differences to 'normal' but rather to accept and to evolve understanding of the aspie world. An effort to diminish their difference may ultimately show their own acclimatisation to 'normal' society.

From my reflection upon the many types of disability discourses and dualisms I can assume that the concepts do not commonly go hand in hand. Few discourses outlined used positive themes surrounding disability, so understandably there exist very few studies of people labelled as both gifted and with aspergers, disability or impairment.

Foucault's (1975) argument was that social positions mark the identities and experiences living under these terms, and that no one is born with any of these labels. Yet social power enables social discourses and identities. People are positioned in ways society chooses for them to exist, inevitably. There do exist, however, conflicting choices and expectations for those labelled as both gifted and aspergers. Living somewhat paradoxically, life experiences may be challenging and a 'juggle' to decide or not to accommodate the social positioning as identities. 
$\sim 66 \sim$ 
$\sim 67 \sim$ 
$\sim 68 \sim$ 


\section{Chapter 3}

\section{Veiled Genius: Manifesting Inability, Masking Talents}

Unusual actions, behaviours viewed as bizarre and certain eccentricity may be assumed by medical or educational professionals and society as indicators of impairment or disability. Differences to social norms may be measured and compared on an ability spectrum, with impairment and 'high ability' on either opposing ends (Biklen \& Kliewer, 2006). On the other hand, prejudgements of people's incompetence's due to overt behaviours may be diminished, if allowed opportunities to reveal capabilities and talents first. This chapter explores the current literature that suggests judgements of ability create measured normalities, and consequently cause negative or disabling discourses.

Biklen and Kliewer (2006) analysed the narratives of people categorised as autistic, who identified their own behaviours and communication as different to what is typical in their American society. The researchers concluded that participants felt that there was little accommodation in their society for display of unconventional behaviours, and they were often subjected to veiled hints about their presumed inabilities. This meant that tasks within their range of capabilities were, in their view, incorrectly seen as challenging for them. Often medical discourses of disability ${ }^{7}$ construct a correlation between perceived physical awkwardness, inability and intellectual impairment (Cowan, et al, 2004).

\footnotetext{
${ }^{7}$ See Chapters 2.3 'Background - Definitions' and Chapter 4: 'Discourse - Review of Literature' 
As one of Biklen and Kliewer's (2006) participants said through typing, his difficulties were "not with thinking and knowing, but with doing and acting" (Bissonnette, cited in Biklen \& Kliewer, 2006, p.181). Doing day-to-day tasks was hard for him as he felt he was constantly judged, and was aware of his struggles. The participant also stated that 'acting' was difficult for him; he referred to his decision to portray himself in a way that was acceptable to society in order to prove he was not different and disabled. Therefore, he appeared to have been challenged by conflicting personal wants - his society's normalities, social approval and his need of belonging, versus the choice to challenge the strong social concept of inability linked to impairment (Biklen \& Kliewer, 2006).

Another more complex issue is how people become placed in positions of incompetence (and often with impairment or disability) concurrently with dualistic labels of gifted or talented - which are by definition associated with non-impaired, non-disabled ability ${ }^{8}$. Again, this is influenced to social comparisons and assumptions about what constitutes disability (Cash, 1999). For example, a participant in Biklen and Kliewer's (2006) study described that after he attempted to socialise by speaking, people got a sense of his inability to do this articulately, and used this as judgements of how well he could perform other common tasks to normal standards. However the researchers revealed the participant's typed communication

\footnotetext{
${ }^{8}$ See Chapter Two 'Background - Definitions' of intellectual impairment, disability and giftedness.
} 
was unique and creative, and that his choice of words may possibly be considered in a traditional 'gifted range' by educational experts.

Other studies and personal narratives have evoked a general preference by participants to be judged instead as potentially gifted or talented (Reis \& Ruban, 2004; Ward \& Alar, 2000). In other words, people expect and want to be judged, positively, and labelled accordingly. This shows the sense of a dualistic nature of ability as well as quests to be accepted within the constructs of power in any given society. For example, personal accounts reveal how people's intelligences were constantly questioned or assumed by others (e.g. teachers, parents, medical experts, employers, peers) as not reaching 'normal' standards. It is these standards that show the working of social norms, with consequent influences upon self confidence and identity (Bagatell, 2007; Sisk, 2003). One personal account revealed:

I certainly understand why I was assumed to be retarded. All of my very awkward movements and all my nonsense sounds made me appear retarded. Perhaps I was. Voices floated around over me. I heard sounds but not words. It wasn't until I had a communication system that I was able to make sense of the sounds.

(Wurzburg, cited in Biklen \& Kliewer, 2006, p.180).

Prima facie evidence of incompetence occurred much less once people deemed disabled learnt effective social norms, such as how to commonly and successfully communicate by speaking, reading and typing. This changed their perception of power relations in their society, giving a sense of respite, escapement of a negatively viewed social position, or freedom from scrutiny (Bagatell, 2007; Ward \& Alar, 2000). For many people in these studies, this was felt as a 'relief' that was sometimes described as a life changing experience. 
Yet Biklen and Kliewer (2006) questioned what would have been the participants' 'fate' or 'destiny' had they not been able to reach these standards of normality; moreover, if participants decided against trying to take part in society by conforming to the 'normal' expectations of being needing to 'prove' (or 'act') a show of ability (Biklen \& Kliewer, 2006). One participant from Bagatell's ethnographic study (2007) decided not to conform to the measures of a considered 'successful' social ability, because the whole ordeal was too stressful. He felt more freedom being himself, as he reported:

Basically, I sort of got off the whole cure mentality because before that's what I was - always wanted a cure... Or I was always thinking of aspergers as like this horrible thing that was like, causing me to have deficiencies and really couldn't function normally, but rather I came to see everything in a different light. Where it was more like, the aspergers is really... integrated into me, my personality is really something you can't separate out. And you know, it was like, I am who I am and, that it should be okay for me to be who I am and what I am... There really is basically no way to teach yourself to be normal. You can teach yourself to appear normal, but you can never really be more normal. And trying to do it is just stressful.

(Participant cited in Bagatell, 2007, p.419).

His apparent lack of social ability meant the young participant had a choice of improving this competency level to a standard of success within his social groups, or alternatively, 'being himself' - but with a perceived consequence of being labelled not normal and perhaps, incompetent. In this sense, his interplay of manifesting inability masks any assumption of talent. Abilities and inabilities influence how people view others and themselves, or as Holland believed "persons look at the world from the positions into which they are persistently cast" (Holland, cited in Bagatell, 2007, p.418). 
According to the discursive framework I am using in this thesis, all abilities can be seen as socially constructed; discourse creates, maintains and stereotypes people into categories, and in turn enables all people in society to take part and use power in some way toward each and all categories $^{9}$ (Foucault, 1977). For instance, the example given above poses further questions, such as how should people judged as nondisabled understand the disabled people they meet? This question rests on the often assumed dualisms and spectrums between disabled and nondisabled people, and gifted and non-gifted' people (Biklen, 2006).

Furthermore, human beings make inevitable assumptions in order to construct categories of people. It is the social force of power that causes social constructs. Discourses present the effects of power, such as the way categories and social groupings are shaped (Fulcher, 1989). However it is the cultural understandings of social groups which lead to prejudiced assumptions. For example, one might assume a person who is labelled 'non-verbal' cannot make any sound and may only understand the simplest of language, perhaps leading logically to an unfounded belief that their cognitive understanding is limited. This example is emphasised by the implications of institutional constructs, such as Special Educational Funding for 'slower learners' at schools or the current New Zealand Invalids' benefits (Office of Disability Issues, 2009). I think this is evident when a non-disabled person speaks to an adult deemed autistic in a patronising way, as if assuming he or she is an eternal child.

\footnotetext{
${ }^{9}$ Refer to Chapter Two "Foundations before the Journey" - "A discursive framework for exploring the asperger world'.
} 
On the other hand, Biklen and Kliewer (2006) furthered the challenge by asking whether people's physical actions are ever indicative of their intellectual abilities. Frankly, when would we know? If a child with autism cannot speak, they are often considered 'below' or 'behind' their peers' intellectual development, as measured by Binet's giftedness theory of life stages, and other social measures (Colangelo \& Davis, 2003). But then if an autistic child, who is unable to speak, displays ability to do mathematics measurably more successful than a trained mathematics teacher, is this considered gifted, alongside verbal impairment? Who decides what and who is competent, disabled, abled, and gifted?

Another challenge is if actions do not necessary reflect thought and ability, what does this say about scholars who constructed the idea of intelligence, as argued by Biklen and Kliewer (2006)? In other words, what constitutes competence? Some authors (Cousar, 2001; Miller, 2005) supposed that physically independent action, including communication and speech remains a component of ability; yet I argue these components are never entirely independent. For example, presenting a talent via artistic methods, can communicate to the outside world the inner convictions of talent one pursues. Bissonnette is one example, who stated

Not allowing people with disabilities their patterns of inspiring art through total freedom of expression is like limiting creativity with censorship. Without art, wafting smell of earth's pleasures would kite away to land of inanimate objects

(Participant cited in Biklen\& Kliewer, 2007, p.182) 
Bissonnette is a writer and artist who had been labelled 'high functioning autistic' (Bissonnette, 2005-2007). His documentary film My Classic Life as an Artist is an example of his perception that being judged disabled is a normal expected part of his life; something he viewed as inescapable (Bissonnette, 2005 - 2007). Bissonnette was categorized by many labels when he was growing up, for example 'moderately mentally retarded', 'schizophrenic', 'clinically insane', 'institutionalised' and 'incarcerated'; during this period he grew aware of how people viewed him (Biklen \& Kliewer, 2006, p.181). These labels often stemmed from the psychologists who tested him formally.

Bassinette's status as 'incompetent' given by the medical profession ruled or destined his position throughout his life. He stated, "Fastening labels on people is like leasing cars with destinations determined beforehand" (Bissonnette, 2005, 2007, n.p). However, when he learnt to type in his thirties, he recalled how people's views of him changed. He learnt the ability to speak and slowly become socially accepted because of this ability. "Expression provided an avenue of acceptance, even appreciation" and he admitted this is an acceptance he could not afford to give up (Bissonnette, 2005, 2007). Yet it was through the normative form of communication that he felt he could escape the negativity of his given social status, position and identity of 'mental retardation'.

What constitutes fairness or justice for people, whether they are labelled, gifted or non-gifted, disabled or non-disabled, or a combination, when they seek to participate in society in ways that differ from the so-called normal 
communications, behaviour or thought? Bissonnette $(2005,2007)$ was proud to identify as someone with autism so long as his other given label of gifted prevailed. His documentary was a unique insight of how someone lived under the terms of self identified 'giftedness and disability' described their life:

Lore around autism uses situations of incompetence to predict what little potential people have to learn creative and artistic skills. Like leading articles in magazines looking at populations of people with disabilities, my aesthetically questionable, but not bad to argue work, is the best way to clear up mysteries of what I am about. This is my reputation with people that know me.

(Bissonnette, 2005, 2007).

Manifestation of incapability may mask talents, if an 'inability' goes hand in hand as behaviour deemed unsociable, unsuccessful or deviant. However, enabling opportunities to express one's own understanding of life may prevent prima facie of evidence of incompetence. This is the essential ingredient of meaningful social inclusion for any person. Lineman called this "'mindedness; if someone is interested in seeking the competence of another; it helps to 'look for it' " (Lineman, 2001, cited by Biklen \& Kliewer, 2006, p.184). This is not so much about discovering or assuming the ability levels of a person, but allowing the opportunity for the person to decide to show their own capabilities in their comfortable ways (Biklen \& Kliewer, 2006, p.184).

Autobiographical narratives offer a window on the specific nature of people's experiences (Biklen \& Kliewer, 2006; Ramcharan \& Grant, 1994). They also give a larger picture of the world individuals inhabit, with views of the ways that people perceive themselves and how they feel they are judged by others. Narratives also give insights into how people negotiate 
different environments and their given social positions in their lives, which are tools for constructing identities (Biklen \& Kliewer, 2006). Narratives such as Bissonnette's $(2005,2007)$ are an arena which may empower people to speak for themselves, and show the importance of their place in other social circles.

Furthermore, narratives offer new possibilities for demonstrating competence. Researchers who created the opportunity for participants to share experiences from the heart meant viewed the participant as a peer with ability. When Bissonnette shared his perception of communication via art, his talent rather than his incompetence, was exposed (Biklen \& Kliewer, 2006). This study incorporates these ideals with other aspies from past literature and present day narratives. 
$\sim 78 \sim$ 


\section{Chapter 4}

\section{Life As We See It: Gifted ‘Aspies” Perceptions of Life}

Narratives offer a good insight into individuals' descriptions of their lives with aspergers and giftedness (Bagatell, 2007). Narratives may be told in a less structured manner, allowing participants to decide on their experiences to share. This chapter outlines the experience of seeking and analysing discourse narratives of other studies. It also explores some of the discourses of their narratives of competence and ability, particularly the dominant medical discourse.

\section{Overview of seeking literature}

Narrative studies about people deemed gifted and disabled people are not common and rather unconventional (Braggett, 1998).The few studies that do exist of the topic are mainly quantitative studies, focusing on the medical and psychiatric domains of how such diagnosis come about (Plomin \& Price, 2003). In order to understand the narratives of people considered gifted and with aspergers, I not only sought research written for academic and medical audiences, but also narratives for general audiences, such as websites, autobiographies, books and articles.

My research is a detached examination of personal narratives. This differs to the reviewed literature of other narratives, as often the general focus of them tends to place the people living with aspergers as experienced expert of autism or aspergers, with regard to their own experiences. This may lead readers to regard their authorship with some accredited authority or popularity. Rather, the narrators offer only perceptions of their realities. I 
choose instead to follow the discourse analysis as conceptualised by Foucault (1977).

Foucault was dubious of "notions of perception seen as a type of truth", especially as discourses may over exert a position of authority (Foucualt, 1973, Parker, 1992). Foucault stated "We must dispense with our habit of looking for an author's authority, and show instead how the power of discourse constrains both author and his utterances" (Foucault, cited by Horrocks, 1997, p.204). In other words, one cannot assume the worldview and discourses narratives reveal are an actual truth, but instead reflect that the experience is the narrators' realities. Therefore, I sought their descriptions of life and power usage to seek discursive meaning within their choice of words and actions, rather than to portray an authorship of absolute truth.

Most narratives tended to use participants who regarded their identities as aspergers, but not gifted (Bagatell, 2007; Ward \& Alar, 2000). This may suggest narratives assumed a dualistic notion that people are deemed as either gifted or not gifted with aspergers, as if emphasising a spectrum of the inability / ability dichotomy. This limits the narratives that are shared. In some respect, the rarity of focussing on participants as tokenistic 'aspies with gifts' places participants in, perhaps, celebrated status positions (Treffert, 2007). This could is an example of the superhero discourse ${ }^{10}$. It creates a sense of people with aspergers and gifts are perhaps 'luckier' to

\footnotetext{
${ }^{10}$ As mentioned in Chapter Two "Foundations before a Journey" - "A discursive framework for exploring the asperger world"
} 
have talent and therefore more successful, than people with aspergers not acknowledged with talents. This can also be summed up by revered 'savant syndrome expert' Professor Treffert,

.... Approximately one in ten (10\%) do have some special skills over a spectrum ranging from what are called 'splinter skills' to 'prodigious' savants. The latter have special skills so spectacular that they would be remarkable even if they were present in nonhandicapped persons. Savant skills also occur in other forms of Developmental Disability, such as Mental Retardation, but with much less frequency, as low as 1:2000 in a residential population.

(Treffert, 2007, n.p).

Unquestionably, each participant in these studies held very unique and varying perceptions of what it meant for them to live with 'dual exceptionalities' (Bagatell, 2007; Biklen \& Kliewer, 2006; Bissonnette, 2005, 2007; Grandin, 2007; Ward \& Alar, 2000). This was due to the wide range of discourses the participants used. Participants were either men or women, over the age of 18 years, and reflected about their lives as early as they were comfortable to. Participants tended to be of European ethnicity and lived in western societies. All had been diagnosed with aspergers or high functioning autism ${ }^{11}$. The fact that all had been diagnosed confirmed to participants the positioning and self identities they used within their discourses and narratives.

A few, such as Grandin (2007) and Bissonnette (cited in Biklen \& Kliewer, 2006) are well known for successful careers of their talents. Others were not famous but willing to share their experiences similarly for the cause of helping others with aspergers. Being accredited with positions of 'asperger expert' meant confidence and pride of identity that narrators were keen to

\footnotetext{
${ }^{11}$ Currently, many people are diagnosed with autism or 'higher-functioning autism' rather than aspergers, as this was a term more commonly used during the 1980's before a diagnostic criteria of aspergers was widely used (personal communication, Greally, 2007)
} 
share. These feelings effectively influenced the discursive language revealed in narratives.

Whilst immersed in narrative analysis, I found thematic discursive patterns. These clearly indicated common experiences within diverse lives with labels of aspergers and of giftedness.

\section{The power of discursive communication and language for}

\section{socialisation}

The ability to communicate in a way that accommodates into social circles was a lifelong challenge for some aspies (Bissonnette, 2005, 2007). Similarly mentioned in Chapter Three, people who appear to have unusual behaviours or impairment with communication, often felt to be judged as having lesser intellectual or academic competence, by people they meet. Examples are when narrators sensed they could not articulate as well as other people.

Narratives of people such as Grandin (2007) and Ward ( Ward \& Alar, 2000) described experiences of feeling that they 'have' an impairment that prevents or limits their abilities to socialize - which suggested that their disability is alluded by a medical discourse of bodily dysfunction. The narratives showed they tended to focus on explanations of why they felt they could not speak in the way their society dictates as normal. For example, Ward described:

Like most autistic people, I am constantly struggling to communicate....My language is quite good, but talking is stressful because it takes a lot of effort for me...I have very few friends because of my communication problems. There are little things 
about the way I move and the tone of my voice that signal to neurotypical people that I am "different."

(Ward \& Alar, 2000, n.p).

Ward said he was aged 2 years when he could 'finally' reach the milestone of talking in paragraphs. He made clear that he continued to be different due to not meeting 'normal childhood developmental stages', as laid out by medical and social expectations in his culture (Ward \& Alar, 2000). The feeling of not achieving due to a condition or members of a social category portrays the idea that social forces of power control and prevailed the actions and beliefs of a medical discourse (Fulcher, 1989). Similar to other narratives, experiences such as this confirmed his belief of having disability.

This contrasts to the opinion of Fulcher (1989) opinion that disability should be judged in a social variation of ability. Along with Bissonnette $(2005,2007)$ and others (Baker, 2007; Rioux, 1994) she believed social factors influence what is considered normal and abnormal abilities. These social factors create opposites of common abilities or inabilities into dualisms, such as communicating articulately or not being able to communicate in a socially approved way (Rioux, 1994).

Bissonnette (participant cited in Biklen \& Kliewer, 2006; Bissonnette, 2005, 2007) explained how he learnt to use facilitated communication and later learnt speaking, and how a 'new world opened up' for him, because he learnt an ordinarily shared task of communication. In particular, that the people of his world viewed him differently, as an independent person able 
to make choices and creatively use the communication tools that others commonly use. Bissonnette stated:

...At Cannes Film Festival it should win award for documentary of tension between affirmation of mental capacity and pain of physical production of language.

(Bissonnette, 2005, 2007, n.p).

In order for Bissonnette to accommodate to social norms he decided to an accept normalities within his world. He attempted to accommodate to society and prove he was more capable than others assumed. However Bissonnette $(2005,2007)$ described that in capabilities should not remain an individual's problem, but encompasses an issue for society.

... and even if one cannot speak nor write, does not verify the mental competence of an individual. In fact, it is a sad misjudgement as well as an influence of the medical discourse that impairments of individuals are an indicator and cause barrier to full participation, and is markedly the problem of the individual.

(Bissonnette, 2005, 2007, n.p).

Some narrators stressed that motivation comes from communicating a need of support; they stated this was essential for a positive outlook on life

with a disability (Rapley, 2004; Tallent-Runnels \& Layton, 2004). Bissonnette expressed his view that disabilities may be changed with the right support systems (Bissonnette, 2005, 2007). This demonstrates a change from a medical discourse to a rights based discourse despite having to accustom to cultural expectations for belonging (Biklen \& Kliewer, 2006; Ramcharan \& Grant, 1994; Rapley, 2004).

\section{Medical models and discourses}

Medical discourses are continually changing, and often narratives portray fluctuations of difference to medical discourse, particularly toward disability. Because medical discourse indoctrinates other discourses such 
as lay or charity discourse, there appear hints of medical discourse. Some narratives mentioned how the minds of people with aspergers function differently when compared to those of people not labelled aspergers. This suggests a genetic explanation of aspergers and giftedness. For example, Treffert shows strong medical discourse when he wrote:

Whether called ancestral, genetic or racial memory, or intuitions, or congenital gifts, the concept of a genetic transmission of sophisticated knowledge, well beyond 'instincts' is necessary to explain how prodigious savants, for example, can know things they never

learned.

Genetic memory factory-installed software exists in the prodigious savant, and indeed... the special brain circuitry of the prodigious savant gives them access to that generally buried potential in spectacular fashion, permitting them to 'know' things they never learned.

(Treffert, 2007, n.p)

Mentioning of medical discourse seemed to transpire regardless if a narrative showed strong slants of rights based disability models. One example is all narrators viewed mental hospital institutions were not beneficial to their lives or any human. From a rights discourse, participants commented that institutions only offered lesser opportunities for recipients; they were insensitive and denied rights to humans (Horrocks \& Jevtic 1997). They felt institutions placed people with autism as needing medical cures and corrections to behaviour. This is shown by the following extract:

No one should limit learning of truth in life to closed rooms occupied only by people with no natural means to communicate.... Going back in desolation where it's only me and letterless walls are not pleasant to think about. Nothing "apartheids" you like the insensitive world of institutional existence....

(Bissonnette, 2005, 2007, n.p).

This is parallel to Foucault's thinking of madness. He argued that madness is a perceived state of mind that is developed by the categorisations that society places upon people as subjects within social relations (Foucault, 
1977). For example, it is not because people are 'ill' that they should or ought to be forced to live in institutions, but because of discursive practices such as alienation that people are 'ill' or 'mad'. This can be best summed up in Foucault's quote:

Madness is an object of perception within a social space that is structured in different ways throughout history. Madness is an object of perception produced by social practices, rather than simply an object of thought or sensibility which could be analyzed.

(Foucault, 1973, Cited by Horrocks, 1998, p.6).

On the other hand, whilst institutions are discussed as ablest and denial of human rights, (suggesting a rights model of disability) narratives portrayed medical discourse in other ways. One narrative employed what Bagatell (2007) called a 'cure mentality'. The narrator in this study hoped scientists might invent a way to 'eliminate autism'. He imagined technology could change his brain, as he explained "why I'm interested... is for, I guess, call it personal reasons... because I want to learn about technologies that I'll be able to use to improve myself' (Bagatell, 2007, p.417). Another example is when Ward (Ward \& Alar, 2000) reminisced how he was aware he was in need of a lot of support and felt he was a negatively different student at school:

In grade school, I spent about half of the time in an emotionally disturbed/ learning disabled (ED / LD) classroom and half the time with general education students. I usually had an aide with me, especially in the general education classes. Without an aide to help me, I would not have been able to learn, because I needed frequent reminders to pay attention and to help manage my behaviour.

(Ward \& Alar, 2000, n.p.).

His choice of words 'emotionally disturbed' 'learning-disabled' ‘ED/LD' and 'general education students' shows dualisms of his abilities compared to those students not needing extra support. He was placed into this position 
and used the dualistic terms from the discourse of the education system his society created (Rioux, 1994). In particular, he stated he needed an aide 'especially' in general education classes, as he was unable to 'manage' his behaviour. These subjective words can be thought as part of the medical discourse, that his disability is part of his body, therefore his problem.

Therefore, whilst some narrators of literature agreed that certain medical discourses are disabling, they continued to incorporate eliminates of medical discourse to explain competence differently. Therefore, dominant discourses such as medical discourse become imposed and contribute to worldviews.

In most cases, a traditional medical discourse was very evident in narratives when diagnosis was discussed, and the terms they used were indicators of a medical description. For example, Parker (2003) was diagnosed as a child 'with ADHD', an adolescent with autism, then an adult with aspergers. This narrator felt that diagnosis did little to benefit him, but rather was used as a way to socially judge and categorise him lower into social status.

Giftedness was mentioned in few narratives, yet did not hold the same level of need for discussion as being categorised as aspergers. Whilst giftedness tended to be more fluid and subjectively labelled compared to the more concrete category of aspergers, the former tended to be backed by traditional discourses of giftedness. This is because impairment continues to be constantly influenced by the medical discourse. When 
giftedness was mentioned in narratives, themes of intelligence quotient tests and genetics were employed. For example, Grandin (2007) described talent in people with autism was due to differences in 'autistic brains'. Grandin suggested that autism is the reason she had fame and talent speaking to animals. This suggests strong notions of medical and traditional worldviews of both giftedness and aspergers, and the fact that ability and competence is continuously measured by these factors.

Exploring narratives of people living with the terms talent and aspergers allowed me as a researcher to reflect on how some people describe their experiences. In particular, I was able to study in a detached manner their understandings of which discourses they tended to use, especially about concepts of abilities. Examining other people's narratives in literature, I was influenced to create casual semi-structured interview questions for this study. This thesis also adopts a participatory method in order for participants to comment and suggest ideas and be part of the research. 
$89 \sim$ 
$\sim 90 \sim$ 


\section{Chapter 5}

\section{Methodology}

\section{Being part of a research community: participants and researchers}

\section{working together}

One of the main aims of this study is to allow the participants' voices to be collaborative in the research process. I feel this sharing of power is a symbol of a celebration of people with diverse ways of thinking and learning, and promoting roles of worth to people living with the label aspergers in New Zealand. Disability researcher and advocate O'Brien (2005, n.p) stated, "The only way ahead is for both researchers and disabled people to work together for new research agendas". New Zealand researcher Lamont (2008) added that collaborative research with participants "involves people most concerned about an issue in evaluating and reflecting on the success or otherwise of their ideas and actions. Therefore, everyone involved becomes researchers" (Lamont, 2008, p. 38). Participatory research enables different opinions of issues to coalesce, and this unique element is paramount to this research. To this end, this type of research promotes an acceptance of all people's capabilities.

This type of methodology continues the human-oriented approach of my research, and follows the very few other participatory research studies with people labelled as with aspergers, especially when also described as gifted. Hence, establishing this role was unique yet challenging. 
Most studies on aspergers and/or giftedness use participants as subjects only - with researchers discursively in fixed status positions of power (Baum, Herbert \& Renzulli, 1994; Cash, 1999).The distinction between the two roles are clear - an active researcher and more passive subject (participant) (Smith, 2008). These roles are usually not negotiable, a discursive normality of the traditional research world. This invariably contributes a discourse of aspergers as an impairment and disability. Hence placing participants labelled as aspergers into shared roles with researchers, shifts the researcher-participant dynamic into a shared collaboration. Oliver said:

The emancipatory research is not how to empower people, but once people have empowered themselves, precisely what research can do to facilitate this process... researchers have to put their knowledge and skills at the disposal of their research subjects, for them to use in whatever ways they choose.

(Oliver, cited in Oliver, 1997, p.17).

If this concept is carried out in research, there needs to be clear role descriptions for both researcher and participants - despite that, often the line between them may be blurred in comparison to traditional research (Gaffney, 2008). For example, Ramcharan and Grant (1994) introduced an alternative and mutually exclusive 'devolved research' paradigm of the following ideas that would enable more research opportunities between both participants and researchers: full devolution of funds to the social groups being studied, and who are in some way normally 'disadvantaged'; allowing participants to negotiate the research agenda with the researcher; creating the opportunities for participants to choose researchers they see as fit and able researchers (preferably researchers also labelled aspergers) or otherwise the participants to undertake the research themselves. 
As researcher, establishing participatory research was a challenge for me. Firstly, engaging an academic ethics board to understand and agree that people labelled as having aspergers are able to be co-researchers meant time and effort. I also maintained strongly that the participants would not be exploited for any perceived 'heroism' or tokenism within or after the research study.

I attempted to plan ways in which any perceived or possible barriers toward participants would be lessened, in an effort to convince the ethics committee that participatory research was best. Yet despite my best efforts, not all of the concepts Ramcharan and Grant (1994) suggested above are compatible with the academic Master's thesis I pursue.

Ramcharan and Grant described their last point as 'empowering the people named disabled' (1994, p.229). Although their rights-based paradigm of disability puts justice at the centre of enquiry, Foucault (1975) would argue that people need not be 'given' power as power already exists within discursive social realms. To 'empower' implies to 'hand over' or 'give permission' or having gained a vice that a person or group held over others. Rather, power is a mechanism that exists in every aspect of society that all people engage in powerful forces in institutionalised societies. As Foucault stated,

Power is not to be read, therefore in terms of one individuals' domination over another or others, or even as that of one class over another of others; for the subject which power has constituted becomes part of the mechanisms of power.

(McHoul \& Grace, 1993, p.22). 
This was discussed earlier in chapter 2 ('Foundations before the Journey'). Ramcharan and Grant's (1994) vision therefore contrasts sharply in that disabled people must have justice by 'taking back' the power that is rightly theirs as equal citizens, rather than the belief of Foucault who stated that all people activity continue the power structures of their societies.

The important point, however, is that by creating opportunities for people labelled as having aspergers to make more meaning of their data, allows research to move into a new paradigm that takes more account of human rights. In this study, my participants and I worked collectively as a team to create meaning. This is important as, like a photographer with a camera and photography work, researchers may impose their own meanings on findings, which could invariably affect the overall outcome of the study (Ramcharan \& Grant, 1994).

Research processes that are disabling to participants

From a social rights perspective, Rioux (1994) argued that the philosophical and moral discourses of citizenship, and equal human rights, are important in the critique of disability research as is any other research on humans (Rioux, 1994). Yet there remain assumptions that people with disability or impairment have a lack of equal status (Fulcher, 1989). This way of thinking is often left unchallenged and unquestioned, despite the fact that undertaking research involving other societal groups or categories of people, would normally be considered immoral, unethical and or inhumane (Rioux, 1994). For example, studies that used a traditional worldview of biological giftedness, in order to study the intelligence levels 
of people of European ethnicity compared to African ethnicity, could be viewed sceptically and racially inferior to other people. In other words, disability and impairment ought not to be a rationale for research that is unacceptable for other groups in society either.

Following from this view, research processes may often be structured in such ways that continue to perpetuate the medical discourse of disability and that implicitly accepts the 'personal tragedy' model (Ramcharan \& Grant, 1994). Examples of this are medical or lay discourses that sought an alleviation of disability, or viewed impairment as a personal problem, rather than assessing how society could change to enable better support and celebrate diversity. Bickenbach (1994, p.54) argued that research methodologies that involved people deemed as disabled allowed participants to move in the social world as a "cultural dope" of the researchers' own epistemologies.

Furthermore, the tacit reliance by researchers on the work of other professional people has maintained the unchallenged categorizations of disabled people in particular ways. For example, studies have used subjects already labelled disabled or with aspergers or autism, as assessed by standards of the Ministry of Education (such as the measuring of 'support need', often categorised as 'very high needs', 'high needs', lower needs' etc) (MacFarlane, 2000). As Ramcharan and Grant (1994) stated,

There is an inherent conservatism in this unexplicated understanding collusive. It is therefore ironic that despite a high degree of acceptance by researchers of the labelling and societal reaction models within social science, researchers continue to be a 
part of, and contribute to, the very system of labelling many of them seek to criticize.

(Ramcharan \& Grant, 1994, p.231).

Other methodologies that have portrayed disablement are when researchers have decided on the concepts used in interviews in advance that may have placed a filter or presented veiled hints about the efficacy of the participants. An example may be by asking participants questions such as 'how does it feel to be pitied by other people?' which may have implied a patronizing tone of 'othering'. Another assumption may be of people labelled as intellectually impaired as also disabled and that due to the disability, the people are to be pitied. Researchers instead need to examine the meaningful social inner world of their subjects from the subjects' points of view. Otherwise, the research is implying the rationality of a researchers' own worldview, which would add more meaning and value than the participants'.

Concepts to develop inclusive research practices are far and few; however my review of participatory research found three themes in research that included opinions of people labelled disabled or impaired. One was the strong belief that researchers who were categorised as disabled or impaired were in the best position for researching people who were also considered disabled or impaired. This was due to the likely shared understandings and sensitivities between participants and researchers. However examples of the employment of such researchers are meagre (Oliver, 1997, Priestly, 1997; Zarb, 1997). This means there remain few disabled researchers who are recognised as able to carry out research tasks. 
The second theme is that there are often limited resources to enable maximum involvement of people deemed impaired or disabled (Priestly, 1997). For example the employment of support people to offer translation of non-verbal or different communication in interviews is limited, and transitions may have consequences of bias or manipulation through translated meanings between support person and participant (Zarb, 1997).

Thirdly, monies have to be secured from the larger and more powerful organizations that plan and implement services and structures, including research arenas (Bickenbach, 1994). This may lead to conflicts of interest between research advocates and organizations. It also continues to place accountability on these powerful groups rather than the participants with disabilities (Fulcher, 1989). Unless researchers and disabled people together take an active stance on disability research, these implications and issues will remain alive and unquestioned.

Benefits for participants with 'aspergers' and giftedness

From my literature review of researching people with intellectual impairment, there are some common benefits, which may potentially enable involvement of people with aspergers and giftedness. This is in light of the limited literature about disabled people taking active roles in research studies about disability.

Firstly, research participants are generally more interested in what and how the research can improve their current situations, rather than 
academic articles or research (Lamont, 2008). Participants are more willing to share life stories if they know the data is used in an advantageous way for participating. For example, using the research as a tool to aide educational strategies or giving a voice for new policies or laws. Bringing together a collective voice (including my own with participants together) means shared understandings of what being an aspie means (Smith, 2008). Therefore the research benefits both parties and has a humanitarian purpose.

People labelled as disabled, impaired or with aspergers have recommended that researchers get actively involved in the person's life, perhaps as a citizen or advocate, or in promotion of self-advocacy. This ensures a close relationship and aids both parties in the interview process, which at times may be personally difficult for either interviewer or participants (Bagatell, 2007). This relationship need not be in an employed professional position, but rather as an active and sincere interest in supporting the participant in some way, such as café visits or walks.

The essential added benefit is a shared language that emerges during the ethos of collaborative research (Lamont, 2008). Carol Gray, writer on the topic of autism 'disorder' stated "the impairment of autism is shared" when both parties work as an equal team (Gray, 1991, cited by Lamont, 2008, p.40). This can be best summed up by Lamont's opinion "those with ASD may not easily be understood by typically developing peers, equally, typically developing peers do not easily understand those with ASD" (Lamont, 2008, p. 40). Moving from a pathological model of attempting to 
make a person with autism to accommodate, an ideal method is to adopt a communication model in which differences of both the researcher and participants are accepted.

Rather than disseminating through artificial mechanisms, the researcher could simply tell the co-researchers / participants the findings and work with them to make any adjustments, with or on their behalf. This process is designed to be fair and equitable for all parties (Lamont, 2008). It allows for areas to be openly discussed and changed, such as errors or adjustments participants make aware to the researcher, or extra details to data.

From these personal accounts of how disability studies is best researched, I can conclude that the emerging belief 'nothing about us without us' is best for working with people with gifts and aspergers (O'Brien, 2005, n.p). In other words, I will ensure that all participants have opportunities to have input of their views, ideas and suggestions and to amend or make clear any misunderstandings of data. I tried to ensure that communication between myself and participants was as consistent and clear as possible, in different mediums and, where necessary, includes non-patronizing 'plain language'. The benefits of taking part in the research were negotiated and made clear for participants.

\section{Beginning the journey of methodology}

The best way to implement fair, ethical and valid research, which incorporates participants' ideas, is in my view, by the use of qualitative 
research. Qualitative research has the benefit of getting broader understanding of participants' experiences and adds ethnological meaning that cannot be found in quantitative statistical analysis. This is the best way to hear participants with aspergers and giftedness' descriptions of their lives, and allows opportunities to express themselves openly.

\section{Case studies}

As qualitative research is voluminous, it tends to work with relatively small numbers of people (Willig, 2001). This is the unique and challenging aspect of holistic case studies. People's lives could then be analysed closely, compared and contrasted with each other. Each participant's story had richly diverse descriptions, which added to the variety of data unparalleled in other types of qualitative research. The use of case studies was therefore ideal, as giftedness and ability levels were so varied, and even more so for peoples' self-perceptions of emotional and social development.

Not surprisingly, qualitative case study research is often criticized for lack of generality (Cresswell, 1998). For instance, the sample of participants I used cannot be viewed as representative of all people deemed aspies. Yet deciding boundaries for case studies was challenging and unfair (Cresswell, 1998). For these reasons I decided not to choose or single out particular potential participants. To do so would place my pre-conceived ideas about giftedness and aspergers and this could produce data that may be an almost predicament. Choosing certain lives of aspies would be 
contradictory to the focus of finding out how talented aspies describe their lives by them, and celebrating their differences.

By deciding against the idea of choosing particular cases, I did not have to struggle with decisions as to whether I wanted 'cases' that are common, stereotypical, exceptional, or relatively 'unusual'. Furthermore, Haug (cited in Willig, 2001, p.49) believed "if a given experience is possible, it is also subject to universalisation". That is, there are always possibilities of unique experiences happening in other parts of the world or time. This is because individuals' perceptions of life are socially constructed, and therefore potentially generalisable.

\section{Gaining entry and seeking participants}

I networked into community organizations, services and educational specialists that cater specifically for people with the labels of aspergers, autism, and disabled people in New Zealand. This involved explaining to gatekeepers about the study and processes, and entrusted them to pass on relevant information via websites, notice boards and word of mouth. I also placed advertisements on bulletin boards. Through my former roles in disability workplaces I found the networking process quite easy.

Recognising that many aspies do not view themselves as disabled I did not limit my support network to disabled organizations. I sought participants in areas they may excel in. University academics, staff and tertiary student notice boards and libraries presented my advertisements. I also networked with staff of the New Zealand Gifted Education Centre, with the hope they would have contacts to former students with of the 
criteria. Whilst these areas were not successful for finding participants, the gatekeepers shared information of other arenas in which I sought for participants.

After nearly 2 months the outcome was that parents, teachers, staff members of non-government organizations and others knew of people considered suitable, yet few actual people with considered giftedness and aspergers chose to volunteer on their own. Those that did were youths or children, or I judged them too young to partake in such a reflective study. I was cautious using referred people as I felt that, as this study was participatory based research, then the right to choose to take part is paramount in the lives of the volunteer participants ${ }^{12}$.

After much reflection, I realised that waiting for people with giftedness and aspergers to come forth was too challenging. A common trait of aspergers as stated in the Diagnostic Criteria for Autism Spectrum Disorders (Ministry of Health, 2008), many people with the aspergers label find social interaction a complex challenge. I had to think 'outside the square'. Hence an assumption was made that for a person with aspergers, approaching a stranger to do research about aspects of their personal life may be too difficult to start a conversation.

\footnotetext{
12 For instance, I was not keen to ask one person to participant because her mother wished her to participate. My intuition was that the person may not have chosen herself to take part. If she had, she might have done so due to compliance to her mother. Subsequently, data had the possibility to be skewed -the daughter may have shared what she felt was expected to share from her parents. It may have also reflected an example of passiveness and compliance commonly seen in the disability world. Therefore, I wanted people to choose for themselves.
} 
I chose a different proactive approach of personally promoting the research study to people with aspergers. A staff member of an autism organization indicated there was a social Adult Asperger Group meeting on a weekend in a city. The adults in this group ranged from early 20 s to elderly and were a mixture or men and women, married and single, parents, academics, professionals and retired. They met together once a month from around the region to socialize and support each other, with an understanding that socializing may be problematic for many members. The convenor invited me to take part in the next event of a shared lunch, and fun activities that enabled socializing for people with aspergers.

Promoting the research in person was successful for finding participants. This was due to a concerted effort to be personable and promote my research amongst the dialogue shared within a specific group. Seven adults took great interest, and were given written information about the study. Some were excited and indicated the decision to take part. Most potential participants stated that there were few research studies for aspies to express what life is actually like for them; rather, what existed for them were studies for parents, siblings or teachers of people with aspergers. This research appeared exciting, innovative and cutting-edge in the eyes of many aspies and it offered them the chance to describe life as what it actually is in their terms. 


\section{Participant selection}

As this is discursive research, the participants were regarded as experts. Their views expressed were their personal views, and not representative of the general aspie population.

Participants were people who identified themselves or by others as having the recognised 'form' of autism called aspergers. I had no intention that the participants be diagnosed, for that would place this study within a medical discourse. However the participants were expected to identity as having this categorisation some time in their life from knowledge of what autism spectrum disorder and asperger syndrome are, since these labels are often found in their social systems. I also expected their understanding to include the known-to-be common but various experiences or issues people with aspergers face, as found in literature (such as diverse talents, issues with socialization and imagination, as well as any unusual, eccentric or unique behaviour) (Autism New Zealand, 2004).

Participants did not necessarily believe they had giftedness or talent but orally communicated to me that they were keen to be part of the study. They understood others may believe their abilities to be of gifted and talented quality. I placed no limitation as to who should decide the level of ability, or what value is placed on a skill, as to do so would be placing an ability or skill under social accredited measuring, similarly as intelligence quotients may do. However, all participants attempted to show me what they felt they were talented at doing, and this reflected in the natural eagerness and pride of identifying as different. 
I chose participants of any so long as they were aged 16 years or older. This was done because parental consent may have meant limitations of participant numbers, or bias from parents' views of who should take part in the study.

Three of the participants, Kea, Eion and Alan were male and Kay was female $e^{13}$. I found very few females who identified or were categorised as 'aspergers' despite my wide search around New Zealand. I found no evidence or research to suggest that females with aspergers where considered less keen or less unlikely to take part in participatory research; instead, my understanding was that because medical discourse seen in literature of aspergers and autism suggested males were more likely 'affected' directs common knowledge of the two, hence supports the few females interested in taking part (Autism Spectrum Disorder Information Network, 2009).

Three of the participants were parents (Kay, Kea and Eion), with one biological child each. These children were also identified as having aspergers, which medical professionals, diagnosticians and researchers might describe as a genetic trait. Whether or not the traits are personality characteristics, commonly shared or 'found amongst people with aspergers is debatable; however I felt the fact that the participants with children also shared asperger traits meant a different and rather unique outlook on aspergers that could provide richer stories for the research.

\footnotetext{
${ }^{13}$ See chapter 2 'Foundations before the Journey' - 'Definitions of autism and aspergers'.
} 
Participants held jobs and Eion, Kay and Kea were married to or separated from partners not deemed by the participants to have aspergers. Participants were of Anglo-pakeha descent and generally were in the age group of 35 to 50 years. (I did not think it appropriate to ask people for their ages directly.)

\section{Establishing rapport with research participants}

I met with people who were keen to be participants to build strong participant / researcher partnerships. Strong relationships were essential for studies such as this, for participants become more relaxed when they shared personal and potentially sensitive information. My trust as a researcher was strengthened and respect shared. This relationship building period took time. I met with some at cafes, at their workplaces, had phone conversations and regular emails. After meeting with me, people who decided and were still able to take part signed consent forms (see appendix).

For each participant, I also became by default, a supportive friend. Becoming a friend had the benefit of being not so much of an 'outsider looking in', but an 'insider within'. That is, I witnessed the great strengths and weaknesses such unique individuals gained in their lives within personal contexts. Descriptions of certain experiences became much stronger once I got to know the participants. Also, I was able to intuitively decide how much the research would benefit each person. I developed an understanding on how to protect each person for ethical and confidential 
considerations. "Harm can occur both during the project and years after its completion. This can arise in avertedly through casual communications about the project, especially if easily identifiable in their communities" (Smith, 2008, p.18). As I had the background knowledge of people's experiences, their personal descriptions of their lives became shared understandings between us.

I did need to use a method of careful selection for participants whom I judged able to understand the consensual nature of the study. This was to ensure that participants understood the dimensions of academic study and could agree to be confidential. This is not unlike other narrative study research, in that a strong trust element is vital. This meant discussing the whole research process with each participant, and being sure that I was able to trust they could relay the information back to me.

The participants were likely to have shared what they believe is their truth and in my view were not likely to have responded solely to please me as the researcher. The participants also asked about the interview questions, sometimes in detail, which indicated their understanding of the research processes and their ability to articulate their thoughts and beliefs about their ideas of giftedness and aspergers, and the impacts these had made to their lives.

\section{Interview procedures}

In designing my interview questions, I felt that I needed to have someone who identified as having an intellectual disability or impairment to help 
shape the methodology. My aim was to understand what would be considered suitable content and wording of interview questions, and how best to ask them. Therefore I sought the help of a professional working in a disability organization, who believed he lived with intellectual disability.

The interview questions were vetted by an advisor. In order to continue the focus on rapport with participants and their own knowledge, a semistructured interview format was chosen with room to ask questions using a conversation method; this allowed the possibility of a more natural and casual manner of communication with a purpose and topic. This was chosen also to minimise social demands for participants who might find social chatter too demanding.

Before conducting the interviews, I carried out a pilot interview with the advisor. The purpose was to practice the conversation method of interviewing, and enabled me to avoid 'leading questions'. This exercise was beneficial to me and allowed practice for better listening and clarifying.

Deciding on key words to use in the study was a challenge for the advisor and me. He, like the disability organization he worked for, adopted institutionalised terms that described people with perceived impairment, such as: 'person with intellectual disability' 'service user' and 'self advocate'. However, during my search for participants, the term 'person with intellectual impairment' was viewed sceptically and crude by some 'aspies' and potential participants, with no respect to whether they actually 
chose to identity as 'having' an 'intellectual impairment'. They felt these terms were decided and controlled by the dualistic 'other' 'neuro-typical' society. On the other hand, being 'socially disabled' or 'socially impaired by society' was considered better-used terms from a few of the potential participants. Others simply accepted the socially normative labels, despite not feeling comfortable with them. As one stated, "It's what's always used to describe us" (potential participant, 2008, Personal communication).

I decided that the safest and most sensitive way to deal with concerns was to speak the language of the participant I was interviewing, incorporating key words at the time. For example, if a participant called themself a 'person with asperger syndrome disorder' then I would continue talking in their manner with their chosen words. As mentioned by Lamont (2008) earlier, sharing a common language is preferred over a pathological model of disability, and allows the experience of aspergers to be more closely shared.

Each participant was shown the themes of the interview questions but not the actual interview questions (see box). The questions were also communicated in an academic written format and verbal communication the aims and focus of the study and the research questions. Participants had the opportunity to make interpretations and suggestions of words and meanings in these communications. It also gave the participants ideas and leeway regarding what they may choose at the time of interviews. 


\section{Questions asked during narratives}

1. Experiences of having a gift / talent:

Question: I've noticed / you have told me that you are very good at (talent). When did you first realise you may have a gift / talent in this way? How did this come up (i.e. at home, with friends, or at school)?

Question: In your life, what sort of experiences have you had due to this talent or gift? For example, how has your gift / talent influenced your experiences at school; work, at home, with friends?

\section{Experiences of having aspergers:}

Question: How did you find out you have aspergers? How do / did you feel about the diagnosis / condition?

Question: Aspergers influences peoples' lives in difference ways. Tell me how you feel Aspergers has influenced you life.

\section{Aspergers and giftedness in society}

Question: How do you view the way society regard you and your needs?

4. Views on other people with giftedness and aspergers:

Question: What are your views on other people who have giftedness and aspergers?

Question: If you had a good friend who has aspergers and giftedness, what advice would you give him / her?

\section{Support network:}

Question: In any areas in your life, who has been supportive of your needs?

5. Sharing personal views about giftedness and aspergers:

Question: How does it feel for you to talk about your experiences living with giftedness and aspergers?

Question: What areas of topics are easy to talk about and why? 
Setting

Interviews were held in a place that was comfortable for both the researcher and participants. One was held in the workplace of one participant, and the other three were held in a university setting. Each premise was in a sound-proof area to ensure confidentiality. For the university settings, I asked each participant if they had any challenges concentrating with background sounds, echoes, distracting visuals or other sensory aspects. None stated they did, except one who asked for a room with a window. This was ensured, as was refreshments and a nonintrusive camera.

Support people were welcomed, had any participants needed or wanted support during the interviews. None chose to use one, perhaps an indication of the strong and open relationship between myself and participants.

\section{Filmed interviews}

I carried out four videotaped interviews with participants. Videotaping was used to enhance the portrayal of participants' communication, including body and facial expressions; this may have also added meaning where written interviews may not, if the participants had any communication issues or differences (Ratcliff, 2004). Some difficulties that had commonly been associated with the diagnosis of aspergers were verbal and social impairment, such as avoidance of eye contact (Autism New Zealand, 2004). Videotaping allowed the recording of such behaviours to enhance 
findings. After interviewing, participants were given a copy of the filmed interview on a DVD.

\section{Participant communication}

Once the interviews were transcribed, each participant was emailed for comments or questions, excerpts, quotes or nuances from their interviews. Each participant communicated back via email and shared extra ideas, suggestions and clarifications for data and the study. For example, if I was not clear about the meaning of a quote, participants would reply with explanations and give me better insight to their data.

Participants mentioned that reading and reflecting on their quotes was insightful for them. This process allowed them to step back and view themselves as others may view them. Participants generally did not change a viewpoint they shared after their interviews but rather added comments on the way they saw them. Some felt this benefited them socially, to understand social cues and nuances.

\section{Trustworthiness between researcher and participants}

I was aware of linguistic variability that could arise during the interview (Minichiello, Aroni, Timewell and Alexander, 1991). For that reason, I sought to understand the words and meanings as participants wanted them to mean, irrespective of how participants said them. This was particularly if participants had verbal or understanding differences. This awareness enabled me to use the findings in a valid and fair manner and to not interpret exploitatively. 
I was also aware of my own concern that participants may have potentially distorted or exaggerated information during the interviews. This may be because the communication is what the participants expected me to receive, in order to enhance their image (Minichiello, Aroni, Timewell, \& Alexander, 1991). However, despite using crosschecking techniques, if is difficult to pin down the truth. As Minichiello (et al., 1990, p.128.) pointed out: "The in-depth interviewer cannot be one hundred per cent sure about the difference. However the researcher is not geared to finding out the truth per se, but rather the truth as the informant sees it to be".

\section{Researcher Bias}

In producing this research I worked hard to remain aware of the personal and professional biases I brought into the study. Smith (2008, p.18) wrote that "When one is researching one's own context, it is important to acknowledge the perspective one brings; however the goal is not to overcome or change this perspective, but later make known how it has affected the research".

I have worked in disability settings and personally known people with autism and/or giftedness. Added to that, I have family members who are classified as disabled. These experiences may have biased my worldview of people with gifts and aspergers as well as what I believed constituted disability, intellectual impairment and giftedness. I felt my biases are

minimized by the recognition of each participant's influence, the completion of written transcripts to preserve the participants' words and 
researcher and participant discussions of data prior and post data collection. However there may be gaps in my knowledge because I am not a person with aspergers or an aspie myself.

\section{Limitations}

There were complex issues involved in choosing the criteria for participants. Due to my upbringing and knowledge of ways that medical discourses construct notions such as impairment and aspergers, I foresaw a difficulty in gathering data, given that people labelled with the name 'Asperger's' are often described as having limitations with tasks involving face-to-face communication, as well as potentially limited ability to understand complex research requirements due to problems such as lack of short or long-term memory ${ }^{14}$.

In my previous work in this sector, I had taught myself to ignore the unsettling communicative tendencies of some service users in former disability workplaces (e.g. limited eye contact). However I did not want to limit my participant search to those with some normative criterion for communication ability, since such a view might be underpinned by a medical discourse, and this would prevent the chance for some unique stories to be confidently shared by participants, regardless of ability. Hence I prepared myself to problem solve creatively, and formulated clear

\footnotetext{
14 This can be common for any participatory narrative research, although, my conscience of what entailed impairment and aspergers from a medical discourse perspective recurred. Limited short-term memory may have meant forgetting what questions were asked, and possibilities of limited long-term memory may have distorted or left out data they could have regarded as important. Whilst no participants admitted either memory issue prior to data collection, I encouraged each to email me thoughts if they felt it best to prevent forgetting, or to keep a personal journal. Two participants chose these options.
} 
strategies to deal with dilemmas that might arise. These strategies included using technology for communication, availing myself of any potential support from participants' family and friends and using the services of organizations mentioned earlier.

One further limitation of this study is that it is small scale, with voices of only four participants; hence, the findings cannot be generalised to represent all people labelled as gifted with disabilities or aspergers. Although there were main themes common to participants' talk, these are limited to the experiences of these few participants.

\section{Data Analysis}

Once data from the interviews were transcribed, I reviewed the filmed interviews, making detailed notes as appropriate. This allowed opportunities to note any behaviour, body language and verbal emphasis the participants showed. It also meant I was able to better understand what the participants would consider as their 'normal' body language for them; i.e. I was able to note behaviour that may appear unusual to another person, but would otherwise had been a regular occurrence to a participant, such as long pauses or avoidance of eye contact. The behaviours noted from video data also added an element of how strong participants felt about certain subjects (e.g., smiling broadly on particular topics).

From the transcripts, words that were in common usage amongst participants were noted, as were unusual or unique words. I used coloured 
highlighting of such words according to apparent themes that seemed consistent between each interview. That is, each theme had an associated colour highlighted on the words of each transcript, acting a code to signify a theme. For examples, the colour green was used for the theme of identity formation. Quotes mentioning the topic related to 'identity formation of giftedness' was highlighted in pale green; 'identity formation of aspergers' was highlighted in dark green. Interesting single or groups of words that appeared to be key terms in participants' meanings were underlined, e.g. terms such as 'high functioning' and 'slightly geeky'.

When each narrative was singly examined each appeared as colourful scripts. However, when compared and contrasted against one another, I could easily view the similar or same colours and thus identify theme consistencies. I then developed an understanding of which themes tended to be more common, strong or unique in participants' talk.

Coloured-coded data helped me to identify the most significant themes and topics and the less important data. This was of immense convenience to me as it meant I could easily obtain the information readily. 
117 
$\sim 118 \sim$ 


\section{Chapter 6}

\section{“'Aspie' is Who I Am"}

From the beginning of each interview, all participants made clear they experienced challenging and colourful journeys growing up with the labels gifted and aspergers syndrome. Fortunately, as participants considered themselves as aspies, their current identities vis a vis the term aspergers were viewed by them as unique and generally positive 'adventure'.

However each participant tended to find upholding the confidence of this identity a challenge. Many participants expressed that revealing traits of uncommon individuality in social situations could be a perceived by others as impairment. This was seen as a paradox, since some of their abilities are recognized by others as gifts or talents; hence, their uncertainty existed around whether their experiences of aspergers was seen as an impairment or a gift, and whether these could be an advantage or not in their lives.

It was perhaps not surprising, then, that being an aspie was described in far greater depth than having gifted talents, perhaps in some ways to justify their uniqueness.

\section{Feeling different to other humans}

Participants shared some understanding of feeling as if they 'don't fit in' to a culture, group, or society, sometime in their lives. They believed this is due to perceived dualistic differences between themselves and neuro- 
typical people. This main theme is similar to studies done by Bagatell (2007) and Biklen and Kliewer (2006).

Participants in this study described feelings of confusion about why they felt they were different to others, and for some participants, these feelings still existed after their interviews. One major influence for participants Kay, Kea, Eion and Alan, was they did not know the concept of 'asperger syndrome'; hence not realising there were opportunities to have a diagnosis, or not knowing the existence of the identity 'aspie'. Furthering these perplexities of identity, participants felt their society also did not know or had limited understanding of autism or asperger syndrome. In other words, they thought that these concepts did not exist as 'real' in their worlds when growing up; and perhaps not in the discourses of those close to them, such as family.

All spoke of experiences where they all felt somewhat different, isolated and lonely, but could not put a finger as to an explanation for their differences.

Eion: Imagine discovering tomorrow ...that you were not of Han Chinese descent, but in fact Miao Yao Chinese, and that you had always known you were somewhat different from the broad sweep of Han ethnicity, that something just did not square in your makeup. But being, at the same time, unable to locate proof of this, definitive proof, access to your culture and community, access to your lore and legends, your special familial way of being, that had been subsumed or lost by other means to the overwhelming numerical and influential superiority of the Han element.

Therefore, until any existed terms, diagnostic criteria, and labels of aspergers and aspie were created, participants such as Eion had little explanation or understanding of their actions and way of thinking. 
Participants believed their differences were likely to do with some intrinsic aspects of their characters, as compared to neuro typical people, for example, Eion's statement, "and that you had always known you were somewhat different... that something just did not square in your makeup". Their differences were often conveyed to participants as appearing unusual in the eyes of people in their lives - whether positively unique, appropriately different, unconventional, deviance, eccentric or odd.

Feeling different to other people may be linked to Foucault's concept of 'madness' and being segregated from others. Horrocks and Jevtic (1997) described Foucault's argument that 'one is not ill internally, but ill due to being separated' (p.7). Participants expressed feeling socially separated due to perceived characteristics that people with aspergers seem to share, or as described by Eion 'squared in their makeup'

\section{Pathway to 'aspie'}

The pathway to learning about aspergers and self-identifying as an aspie was different for each participant; yet all experiences involved learning of other people they knew as having aspergers early on. Whilst participants had not heard of the terms aspergers or aspie when younger, they had heard of the medical description of autism and personally knew people with autism who required a lot of support to learn.

Adulthood tended to be the time when participants learnt of the term aspergers. Being adults, participants had opportunities and decisions to discover the world outside their families. This period may have happened 
at a time that the term aspergers become more socially known in the mid1980s when diagnostic criteria were being formed, as according to participant Eion. Eion and other participants' understandings that autism may be an impairment or disability, and that aspergers is considered a related type of autism, meant they had complex reflections upon their identities. Learning of the terms aspergers 'syndrome' and aspie became turning points in their life journeys.

Kay: well I'm ... l'd say l'm probably on the aspie scale... certainly reading the book 'Pretending To Be Normal and... 'Woman From Another Planet', there was so much of the experiences of the women in those books...

Eion: Well I had always known. When I was 18 I went to 'Handicamp' and looked after a boy with classic autism called Dylan, and Dylan was like me but further down that track [arm gesturing a large length]. I could see, I could see he was like, aloof, closed off communication wise, and I was a little bit at that age as well

These comments show that these were turning points in Eion and Kay's lives. Finding others they could relate to meant they felt less isolated and that other people may understand their differences.

The participants who are parents shared experiences learning they likely had aspergers as their sons were diagnosed (either by a professionals or considered by themselves as parents), and learning from medical experts or literature that aspergers is believed to be genetic. Having family members with aspergers tended to make understanding of the term for participants less lonely; for example, understanding the shared family behaviours usually considered 'unusual' by others, meant participants had explanations to describe the differences to other people.

Eion: it was only when my son was diagnosed by a paediatrician, 
at 3 years of age. He said "Luke, like his father, has aspergers syndrome

Kea: The journey was.... Uh, the first understanding of it... my brother, he got his oldest son diagnosed.... and then, when they came up to see my parents and his wife they said (to me) "oh! You look a bit like, a little aspergers too!" [Laughs]

The above quotes indicate ways that the families of Kay, Eion and Kea went through phases of learning about aspergers as criteria became better known. As other family members become diagnosed, Kea assumed he too was an aspie. This identity felt 'natural' for him, and he stated the word was really a defined title to an already close-knit group that was part of his life. On the topic of his family having aspergers:

Kea: Yeah they were seen as a little bit different and if we were (aspergers), our extended family, it's like, it was 'normal' [laughs]... and, um, it's not something that you'd sort of questioned

Kea's extract gives a description of what an ordinary life can be like for an aspie, compared to the majority of people in a community who are considered 'normal. Kea was raised in a family where all the males were eventually considered as having 'asperger' traits. He described his father as hardworking, well known and highly respected in the community as a religious leader. As a result, people outside the family did not challenge the family's differences that may have been obvious to them, perhaps viewing his higher social status and success as a reason. Kea and his family had a way of living that suited him and this meant an acquired, seemingly natural 'normalness'. However once he left home and socialized with a majority of considered neuro typical people, his differences felt more obvious to him. 


\section{Diagnosis of aspergers}

Diagnosis was a major aspect of helping participants understand themselves and explain their differences to themselves and others. Being medically tested as this category was important to some participants, as the outcome of diagnosis would inevitably change their life courses. Some participants decided diagnosis was the key to understanding their world, for themselves and for everyone in their life. On the other hand, diagnosis existed as an option that could not guarantee better circumstances to life, and had the potential to create consequences that were disabling or limiting. Despite the fact that I posed no questions surrounding diagnosis, the topic of diagnosis of aspergers frequently featured as a major influence on participants' lives.

As none of the participants were diagnosed as children, decisions surrounding diagnosis of aspergers as adults was a taxing issue. For some participants, their differences as aspies were reinforced since choosing diagnosis. Participants relied on medical experts to be sure they had aspergers, for example, Alan explained he consulted a psychologist, whose report "pretty much says it". Participants' instinctive feeling of having aspergers often existed prior to diagnosis had they heard of the term, but having formal testing meant an assurance to themselves and other people.

In other words, diagnosis also confirmed identities. Alan and Eion believed getting a diagnosis early was 'absolutely essential to survival'. Early diagnosis confirming identity meant longer periods in life to grow used to 
the identity label, getting essential support early and living a comfortable life. Diagnosis allowed participants to prove to other people their differences and had a description to back up their explanations. It also meant aspies had something in common and diagnosis created a shared social group.

Interestingly, my literature search reviewed no discussion of the idea of participants deciding to have a diagnosis. Studies using personal narratives or case studies (Bagatell, 2007; Biklen \& Kliewer, 2006) suggested that diagnosis had happened already in participants' lives, especially in childhood, and that there was no choosing to be diagnosed . I assume, then, that those sharing narratives in other studies were chosen because being diagnosed meant they 'were definitely aspergers', and then considered as participants. This therefore continues medical discourse in such research, despite some studies stressing a need for a more rightsbased discourse in disability studies. If this were a goal, then diagnosis and medical categorise would be less important, with less reliance on medical language unless mentioned by participants themselves.

In this study, the decisions of Alan and Eion to be diagnosed signifies participants chose, needed or consciously relied on the medical discourse of what constitutes aspergers, autism and impairment. Diagnosis therefore constituted what is already known and assumed in society; that is, an assurance that something is different and that 'something' exists as a name or label. The diagnosis also exists as believable to participants because of the pervasiveness of medical professions in their lives. Hence, 
diagnosis framed within a medical discourse organised the thinking and inner belief of participants about the concept of aspergers and is an example of how professionalism of impairment and disability can shape the understanding of one's lives. The importance of medical staff in participants' lives is shown below.

Kea: I needed a comprehensive idea of what was going on and... um, I needed to talk to a psychologist um, about this, you know ... and I saved up money and went along and saw [psychologists' name] ... as far as I'm concerned it basically says 'yup I'm on the spectrum'... I don't have to sort of prove it to anybody, I don't have to say 'oh I think I'm on it but I'm not sure'

Eion: If only I knew back then what I know now, but I am one of the few who can honestly say that diagnosis, early-diagnosis, would have meant an altogether better and more pleasant life, that diagnosis would have been a key to so many strategies, and it would have been a reason for my behaviour and that of others towards me, not an excuse for my behaviour, but a portal to understanding.

Diagnosis meant an understanding of their differences to them and society in general, and led to a hoped for social understanding and empathy rather than 'excuses' for their differences. All participants had shared experiences of being incorrectly and perhaps ignorantly described as defiant, or unusual, by family members, partners, friends, teachers and employers within their lives. Using the explanatory of diagnosis, of aspergers as a category or identity, allowed participants to make sense of their differences and describe them to others.

Participants felt that admitting an identity or diagnosis of aspergers could be a challenge if experienced in a stressful social situation. Kea described a constructive dismissal process at his place of employment due to his 'unusual' behaviour at work that was viewed by superiors as unproductive. 
He felt he needed to organize a diagnosis to add to his argument that his behaviour was due largely to having a mind that thinks differently. A diagnosis of aspergers was a last resort in his situation:

Kea : it was actually pragmatism we actually got it, because it looked as if it was a constructive dismissal process going, or starting... at work ... but uh, the reason was to actually force them to do a second take... To have it (a diagnosis) was... [pause of longer than 1 minute]... a not quite a 'get out of jail free' card ... um, I don't know if it actually, actually changes people's attitudes to you...

Following this incident, Kea's employer presented a power point presentation on 'What is Asperger Syndrome' to colleagues. This was designed to educate staff on the diverse ways Kea learns and socialised and how staff could accommodate to his differences. However Kea needed to have the diagnosis to confirm his difference, and justify his actions in order to remain employed at his workplace.

Kea's experience was one shared by several participants: that diagnosis is not always beneficial to their lives, nor does it make life any easier or more complete. There are many reasons for this. Diagnosis comes from the medical discourse of disability and furthered the social stigma obvious in vital arenas of their lives, such as employment or relationships, as Kea described.

Participants spoke of how they felt they were often viewed at first glance as belonging to a group of 'disabled or impaired people' and secondly as individuals. They felt society did not see past the label aspergers, which to the participants reinforced the idea that this is was an intellectual impairment. That is, the label inevitably meant being treated as less than 
able, and being judged further from the norm by difference of behaviour, language and thought. The label increased their feelings of social isolation and loneliness and did little to assist in life. Such a discourse conveyed a blaming or deficit attitude toward participants.

Medical discourse permitted a comparatively objective 'removed' medical language and that language may have impacted largely on how aspergers is understood within society. Similarly to other narratives about aspergers, how people in participants' lives viewed 'aspergers' strongly influenced their own beliefs about their identities, and appeared to be extremely important for social survival.

Kay viewed the medical discourse as offensive and decided against a diagnosis. She also held strong beliefs that admitting her and her son has aspergers would continually be viewed negatively by others in her social circles. Kay's email described how her and her son decided to not have diagnosis for this reason:

Kay: I asked my son if he wanted to be diagnosed, and he said no; because while there is tolerance at his school of ASD people they are still regarded as different and they are not treated the same as everyone else. He didn't want to be labelled 'special'.

Kay followed her argument by stating that diagnosis was viewed by her as a practice done to discriminate against people into social ranks and only added to the current ablest negativity so portrayed in society.

Kay: All of us (friends identified as aspies) dislike the $\mathrm{D}=$ Disorder part and are not going to submit to testing for someone else's benefit. I think there is a feeling of anger with some Aspies, about pressures to conform and to do the group thing for the sake of someone else's peace of mind. 
...I have heard stories from co-workers... about the weird things their rellies do. Sometimes the behaviour sounds quite sensible to me, but the laughter, pity \& sympathy are clues that my views are not shared. Sometimes I say something 'odd' (to them but not to me), and get a pitying look or a patronizing reaction. I imagine that I would get more of that if I self-identified all the time as Aspy. I don't want pity or hand patting. I'm happier identifying as weird, as a geek, as someone different, than as someone with a disability who needs to be constrained or protected.... but not having a diagnosis makes it less of an issue. It's like gays in the US army under "don't ask, don't tell". I don't think telling too much would be helpful."

(Participant's emailed communication in original prose).

One can see from the above comments that to choose to have a diagnosis was viewed differently by participants, as simply not beneficial, or very beneficial. The usefulness of diagnosis for some participants such as Alan and Eion, was to understand about the condition and to have clarity or reason for socially perceived variances of behaviours and living. For Kay and Kea, choosing to not have a diagnosis prevents them from conforming to an already disabling world, or being ridiculed or not taken seriously. However, in Kea's case, diagnosis was necessary as a way to hopefully explain and prevent difference.

Despite participants' different reasons for choosing whether or not the participants choose to have a diagnosis, getting one has never made any participants feel complete or have less complicated lives. In Kay's case, she argued that it would subject her to further disabling factors, such as bullying in the workplace and a sense of being patronized. Kea felt the diagnosis furthered his difference, as viewed as an unproductive staff with unusual actions. He felt less accommodated within the workplace, and instead subjected to a distancing of being uncommon. 
Therefore, the categorisation of aspergers created a 'crisis', in many ways, as well as a 'cause' to explanations of unusual differences. Whether or not the participants choose to have a diagnosis, the identity of aspergers and aspie had been a challenge to accept, but often came with relief. Without the label of aspergers, it was hard for participants to identity as a group in order to make sense of their world. At the very least, 'aspie' existed as a term which reminded people identifying as this that they belong, as Donna Williams (1992) describes in her book Nobody, Nowhere. Feeling lonely or discriminated against in other aspects of social life often resulted in a desperation and human need to feel accepted within a category of sorts (Foucault, 1973).

With few adults diagnosed in New Zealand, some participants have believed they have been misdiagnosed or received dual diagnoses of disability and 'mental illness'. This again follows the medical discourse, as the language used shows. Some common diagnoses were 'schizophrenia', 'panic disorder', obsessive compulsive disorder', 'anxiety disorders' and 'attention deficit hyperactivity disorder'.

Having a dual or misdiagnosis may have happened for many possible reasons. Dual or misdiagnosis of a mental illness may be partly due to what Autism New Zealand (2004, n.p.) described as 'co-morbid disabilities associated with aspergers and autism'. If one is socially stigmatised as 'strange and eccentric', feeling a sense of not 'fitting in', or being labelled intellectually impaired, mental health may suffer as a result. The mental 
illness characteristics may be more obvious to a diagnostician than traits of aspergers.

Another possibility is that the participants' talented abilities were not considered 'disabled enough' to associate with intellectual impairment. Often the participants' relatively common life experience and abilities may be enough to be categorised as not intellectually impaired. For example, all participants learnt at regular schools as opposed to 'special needs' schools for students who receive funding due to disabilities. Eion, Kay and Kea had been, or are married; all participants have been employed. This supposition is due to past literature surrounding this issue of misdiagnosis (Biklen \& Kliewer, 2006). This medical discourse assumes then that disabled people may not or have these experiences to the same extent due to impairment or disability. In fact, not appearing physically impaired can hint at normalcy, alluded to when Bevan-Brown states, "Children with difference on the ASD spectrum are often referred to as having a 'hidden disability"' (2008, p.30).

However, aspergers as a concept, had been viewed as a disability as seen by the Kea's statement about a man with aspergers picture in the newspaper for accidentally being in trouble with the law:

Kea : I can kinda understand his case but it's like [mimics the man] 'Uh Me - Asperger' [gesture by pointing to self].

[Mimics the neuro-typical judge:] "Ok but I'm still going to judge you as a normal person"

[Kea shakes his head] Well he doesn't look any - he doesn't even look disabled! 
The concept of 'disabled' in this quote demonstrates the cultural and social implications of medical discourse to structure people's membership within a socially organized group or label. The fact that Kea also describes the young man as an aspie and judged by the courts as inherently different due to aspergers but 'not disabled enough' to be judged as intellectually impaired, shows he discursively continues a normative medical worldview of disability and aspergers. For example, his description of the man who did not appear disabled suggests that despite his own feelings of preferring to not be diagnosed due to social stigma of aspergers, there remains a social stereotype of a disabled person, and that aspergers is a type of disability.

Other reasons aspies might be diagnosed under the 'mental health' system are the rarity of a doctor / psychologist who is knowledgeable about asperger syndrome, especially those understanding that this impairment may not be an illness. Due to the relative newness of diagnostic criteria, the diagnosis of aspergers for adults is expensive; furthermore there are very few psychologists available who can give a diagnosis.

Such misdiagnosis or dual diagnosis is believed by participants to have influenced negative 'down-turns' in their lives, and feelings of lack of self control. This lay discourse is seen when diagnosis of mental illnesses came with medicines and medical support that was not always welcomed by participants or helpful to their mental or physical health. Due to participants getting lost in the identity journey of aspergers there consisted 
an intermingling of confusion, many medical and social labels, and searches for identities.

Alan: I've had descriptions of descriptions of symptoms... there's been obsessive-compulsive disorder, some behavioural ... thing, panic disorder... and anxiety. I didn't understand why this - sort of 'crème or clump' if you like of (mental health labels)... aspergers really gives me that option because up until then I talked to my GP... (But) my GP new nothing about autism, apart from Kanner's autism

Kea: I'm busy on identifying on it (aspergers) because... it's been a journey for me. I actually got admitted to Wellington psych ... for a number of reasons anyway I, um, got a tentative diagnosis of reactive psychosis but I don't know if it was actually real psychosis because, because, because I think it was more like A-typical

Cam: so do you think this was before you knew aspergers was known?

Kea: yeah this was 1992

Cam: [clarification] are you saying they just gave this label because that's what they thought, or...

Kea: well the way I think about it now, is that you can easily diagnose the presenting symptoms

Over time, after undergoing treatments and medicines, participants questioned the idea of having mental illnesses. Some learnt of asperger syndrome from meeting other people diagnosed within this category. Participants seemed to have a conviction that an impairment of aspergers seemed far more plausible a description of their behaviours and thoughts, than illnesses with focuses on rehabilitation. The search for answers and an identity was paramount. From the difference between congenital impairment and mental illness, many felt quite betrayed that presenting symptoms was used as diagnosis of mental illness.

Eion: Oh, how much suffering I could have avoided. How many better streets I could have travelled to success instead of banging my head against the wall at the end of so many dead-ends... 
Yes, I know I am not the only person to be chanting at my age "if only I knew back then what I know now", but I am one of the few who can honestly say that diagnosis, earlydiagnosis, would have meant an altogether better and more pleasant life, that diagnosis would have been a key to so many strategies, and it would have been a reason for my behaviour and that of others towards me, not an excuse for my behaviour, but a portal to understanding.

\section{"Being 'aspie' is optimistically unique"}

Admitting to the aspie identity within a neurotypical world appeared to be a mission for some participants. This often came from the preceding major experiences of deciding to be diagnosed by a medical expert, or do a selfdiagnosis. The interplay of being part of the common social forces of power, or making a decision to journey against the social norm was huge for all participants. Participants all described their resiliency in dealing with stressful and challenging moments when other people believed their behaviours were unusual or inept. These experiences are also shared in other disability narratives and studies, particularly the 'hidden disability' aspergers (Bevan-Brown, 2008, p.30). However inner convictions meant participants are able to live with the identity in ways that are optimistic, as shown in the excerpts below:

Eion I looked upon it as both positive and negative at first, because... negative, because it was something that I was, but hadn't known and had needed to know before... and it was upsetting... but also positive in terms of I automatically looked at it also as the key that would unlock the story of rest of my life... and a lot of people never get that opportunity... and it was something I was very keen to find out how to do things different.. uh why am I so different? and I learnt that difference is not a good or bad thing, it's just a difference and that there is a different way of being... and it's a lot happier being different instead of just fighting it all the time... which I don't bother any more now... 
Kay: For me because l've gone through so many areas of my life of self identity and other things I guess it's a progressive cascading...so like I'm out as bisexual, I'm out as pagan and have faith... I actually go to about 5 different - groups from time to time...

... I guess I am old enough and secure enough that if anybody is going to get negatively affected by it - tough that's their outlook, not mine.

So (identifying as aspie) it's also a gradual thing... probably if I got really negative responses from somebody, you know, I might sort of crawl back. But I sort of mention things a little bit progressively and you know... that's fine. But it's not like being as fully out... I probably wouldn't wear a badge...

Kay and Eion's examples show how confusing the journey can be identifying as aspies. The journey is fraught with accepting the term aspergers as a socialised group, and understanding how their social positioning is related to neuro-typical people. Their pathways to accepting their identities of aspergers were challenged by judgements made by other people. However their choosing to accept the identity came with the confidence of knowing other people exist with the same identity.

All participants shared an understanding that being classified by society, as aspergers was something that is unchangeable in their lives, as their socialised worlds continued to use this measured social grouping. Whilst this is debatable, this is also backed by the 'disability' studies reviewed in chapter three. Other social classifications they lived under may change, but aspergers was unlikely to be one of them. Hence participants took the shared view that to identify, as aspie, could be a choice and decision. With other successful role models, aspie was viewed as a rather positive characterization, having been discursively developed by and for aspies 
themselves. It may not be their only identity, but leads a relatively large aspect of their lives. 
137 
$138 \sim$ 


\section{Chapter 7}

\section{Sharing the 'Asperger' World as 'Aspies'}

Participants developed aspie identities when they discovered that other people proudly identified as having aspergers; hence they developed the coined term 'aspie'. Participants were full of energy when they described ways of seeking others with aspergers to contribute support and grow shared understandings.

Power structures created by society influence a human need to be part of a group (Foucault, 1975). Other aspies mentioned in the participants' narratives helped create distinct commonalities that are unique to them, and also empathy and support within a world of mainly misunderstanding 'neurotypical' people. This world may otherwise be adverse to unusual differences.

Typically, dialogue surrounding having a supportive social arena with other aspies lightened the participants' narratives:

Eion: They (aspies) are the neatest people in the world... I wanted to spend weeks with each one, I would have been so happy... it's like coming across the most amazing person, and then having to go onto the next appointment. Unfair that. Intellectually, they tickle me. Somewhere deep inside my head and it's a really nice feeling being with them, very comfortable

Alan: We're sort of like locked in - oh I don't know... locked in a sort of... well... the guys I get along with... we are comfortable with world facts, world real facts with absolutely no threats whatsoever...It's when it comes around to things like intimacy, closeness, reassurance, sexuality, we are very, very frightened. We are, we are, well, I'm very frightened... 
and a lot of that comes from thinking you already have a job or position within society...

...Regular folks I find a little bit difficult and very, very challenging ... you know, I know I can trust Allison... and Brendan, and James, and James knows Allison... we speak the same language, you know? [Smiles]

Participants may have led fairly constrained lives if they had lived without the support from being part of the aspie social group. Feeling 'locked in' as Alan described, existed as a feeling of being in solidarity but yearning for friendship. Had the participants not known others existed with aspergers, or aspie identities, their pride of being aspies might have been markedly lower. 'Speaking the same language' as Alan referred to imply a variance to common social systems that only members of this social group understand, with compassion. For example, words such as 'neuro typical' and understandings of social cues seemed to be understood within the aspie community.

Age was not an issue for participants; having knowledge that there were others similar to them made life seem less confusing, less stigmatising and less lonely. Eion described to me a child he met when he was in his teenage years, the first person he felt connected to by his categorisation of an autistic spectrum (and first understanding of autism).

Eion: I thought he was the neatest kid I had ever met in my life and... he was just so unthreatening and so quiet and so all of those things that I like in another person... that I just felt drawn towards the way he was, drawn towards the difference that he had and, um, I felt a bond, an ability to understand what sort of weird thinking path he was on

Eion's excerpt shows the similarities he felt he shared with this boy, and the vast differences between themselves and a common society. Eion's 
choice of words "weird thinking path" demonstrates that Eion understands the way he and the boy with aspergers thinks and lives may be considered unusual by a majority of neuro-typicals.

Feelings of being judged and not understood seemed to go hand in hand with feelings of powerlessness and isolation within a social system. This is parallel to the theory that social power forces create the negated social status of asperger 'syndrome'. Similarly, narratives from literature (e.g. Bagatell, 2007; Grandin, 2007; Ward \& Alar, 2000) suggested lower social positioning influences feelings of confusion, loneliness and a need and want to find someone in similar situations. In other words, without other aspies to share support, participants felt sure they may have 'suffered' from a deprivation of being wholly understood by other non-aspie people.

As with all social groups, there do remain those who are more revered, or experience periods of celebrity status within the aspie world. This is because the aspie social group is, like any other, a community of sorts. For example, Kay was considered as a 'gifted child' by adults, as she used polysyllabic words at aged 2. Foucault (1975) and many others would argue that social statuses exist within all communities. Donna Williams, Temple Grandin and Tony Attwood are a few celebrated aspies, considered successful role models by some of the participants, and were sometimes mentioned as is shown in the next section.

In fact, all aspies are labelled and positioned within their membership and community. Often or not, aspies and people with aspergers measured 
status of 'ability to succeed' depended on how much accreditation they received for being able to 'survive' within the neuro typical world as aspie.

\section{Learning how to survive socially}

The hunt for a 'global aspie fraternity' seemed to be a shared life goal for participants, especially so, to learn from each other how to survive socially. Participants had actively taken part in personally supportive arenas such as online forums, chat-rooms, blogs and websites. Modern day technology tended to be seen by some participants as a lifesaver. For example, some participants mentioned having pride in reaching out to other people with aspergers who struggled with aspie identity and decisions surrounding diagnosis. Some participants enjoyed researching non-profit organizations that support people with aspergers. Kay explained her experience of reading books:

Kay: I bought that book by Temple Grandin on social unwritten rules... (it) actually helps to explain what the rules are for certain circumstances coz a lot of people l've encountered are people who may be on the... aspie spectrum.. (They) are more comfortable if there are rules to operate

The extract is an example that feelings of not being accepted as an aspie were strongly felt, nearly every day of their lives. There were various coping mechanisms that aspies created in order to remain proud of being an aspie whilst enduring hardships. This details the enormity of how the social force of power within a community and 'fitting in' can interplay with a positive recognition of self. It also illustrates the often demanding, and sometimes self destructive challenge of conforming to social norms and expectations.

Kea: everyone is saying "here's the position, here's what is expected" [high pitched mimic] of me...uh, "we realize you 
need to accommodate, so you need to accommodate to our" - or their "culture".

And I think, they think [mimics voices] "you should be 'happy' to be accommodating to this culture"...and it's like - uh, why? (Italics added).

Alan: you'll see you've got to develop a certain sort of, how would I put it, 'rugged', uh, eccentricity, or more self determination... to survive.

Perhaps, deciding or subconsciously appearing as eccentric may allow participants to be true to themselves as proud aspies with unusual behaviours. This choosing possibly means they could be proud of being unique, as well as not appearing nonconforming or deviant to the rest of society, or needing to be treated differently. As Kay mentioned earlier,

Kay: I'm happier identifying as weird, as a geek, as someone different, than as someone with a disability who needs to be constrained or protected.

Having coping mechanisms may have meant the participants were aware of aspects of medical and lay discourse that so many people in their societies live in, such as words and their often negated common meanings. Perhaps they understand the nature of these discourses so plan how to manage their situations, such as Kea's understanding of his workplace's expectations of him fitting in. Therefore coping mechanisms may be a way of dealing with identifying as 'normatively different' but yet still managing to 'fit in' with the common ways of society.

\section{Positive advice to other 'aspies'}

Participants certainly believed that people with aspergers could truly succeed if they were taught what to expect in society by other self identified aspies, and this would enable them to experience life adventures practicing the advice and wisdom shared. Often the advice participants 
wanted to give people with aspergers was to prevent pitfalls or mistakes done on their part, avoid embarrassment and possible experiences of being ostracised from society.

Sharing these coping techniques with others become such a phenomenon that participants had taken opportunities to read and write ideas from and for other aspies. Eion, Alan and Kay gave experiences working in nonprofit organising that benefit the rights of aspies. Many of their roles were done passionately and voluntarily. Participants' life goals seemed to be reassuring others with aspergers that life can be positive, challenging and fruitful. This follows on from the celebrated works of Donna Williams (1992)

Alan: I have a friend whose... son has been diagnosed as being asperger syndrome autistic... and I said "hey that's really the neatest thing you could ever want for him"... and she said "why?" and I said, "well he's four now, going onto five, you have the option of taking him to a really good school if you want, checking out the schools you think are going to be supportive of him... by the time he's 20 he'll have had 16 years of real, real focused attention on how to make the best of his, uh, unique abilities... you know, there's a lot less catching up to do". Me, on the other hand l've got like 25 years. [Frowns] Oh I didn't realize... how much (it) is to learn to be social... yeah, yeah and if you've got the social skills you're all right

Alan's advice indicates that caution for socialisation is often a vital thing when making decisions for supporting people with aspergers. He mentions seeking out the schools parents believed will be supportive of aspergers. This implies that in his reality there were arenas where people with aspergers were not well accepted or tolerated. With support from family, Alan believed the school would focus instead of 'how to make the best of his unique abilities' rather than dualistically focus on what the child cannot 
do. Therefore, Alan's advice is two-fold: help find suitable environments that have good quality support for socialisation, and at the same time be aware that there may be unsuitable or challenging social arenas for people with aspergers.

Finding a job or career was often a topic that participants wanted to help other people with aspergers to do well in. This would mainly be how to socialise in order to gain work, or how to use abilities in ways that would be best noticed in the workplace. In this way, differences in learning and socialising would not be focussed as incapabilities or errors of judgement. The creativity of participants' approaches can be seen in the discussion below.

Kea: look for a niche. Look for a crack in somewhere and ... look for... inclusive employers. Look for clues that an employer uh, tolerates diversity

Cam: Do you think many employers do that?

Kea: my one doesn't really, um... [Thinks] and it depends on your culture too

This excerpt is also demonstrative that society plays immense parts on people with aspergers' lives, and acknowledging this seemed vital to Kea. Similarly to Alan, people with aspergers may be caught in an obstacle should the culture or society not accept or tolerate diversity such as aspergers. Therefore, people with aspergers may feel they need support to seek arenas which are measurably safe and less of a barrier to social participation. Participants stressed people with aspergers should also endeavour to pursue arenas that are non-ablest themselves, rather than allow or wait for society to demonstrate awareness, accommodation and 
understanding of 'aspergers'. This would improve personal confidence and pride.

\section{Pretending to be 'normal'}

Whilst participants felt a growing optimism for identity and helping other aspies learn the social rules in life, there do remain cautious 'do's and don'ts' for socialisation. Yet, the advice tends to be on how to 'survive' by 'acting normal'. In this sense, the rights-based outcry of accepting participants for 'who they are' may be contestable and contradictory if, in some instances, the advice is to 'act normal'. One illustration is Kay's recommendation to 'hide' any obvious traits that would indicate socially perceived 'negative' difference, such as aspergers.

Kay: Camouflage. It's about how to be an animal, a prey animal in a forest... and if children are sort of interested in animals they can sort of follow that... [Thinks] ...it's like, how can you hide? How can you pretend to be normal? Just to get by on a day to day basis?

Kay's past success with employment and friendships may be measured upon this discursive 'masking of inabilities'. However, Kay's concept of masking aspergers could potentially be entwined with further challenges. Because of the mixed consequences and spontaneity of life situations, other people with aspergers being aware of such recommendations may feel a sense of wariness, confusion or finding the whole process taxing. In the same excerpt, Kay also agreed that hiding is not so easy but neither is 'being normal'; insofar, pretending to be 'normal' could have a consequence of believing oneself as not proud to be an 'aspie'.

Pretending to be normal places the added contradictory stress of being successful as an aspie within an asperger world - and the success is 
viewed by many as having an ability to 'fit in' successfully in a neurotypical world. As mentioned earlier, within any community, there exist social status, and many 'aspies' are revered by aspies and people with aspergers because they are fairly successful accommodating into both worlds.

Sharing an aspie world can be exciting and creates a bond between likeminded people with aspergers. Advice and support can be shared, such as learning how to survive and pretending to be normal. These creative options may help in areas such as employment and friendship. However, hiding aspects of aspergers suggests a perhaps lesser confidence in aspie identity. The words, meanings and understandings shared about aspergers in the narratives, and possibly amongst each other, depict a somewhat contradictory nature for aspie identity and the world of aspergers. 
$\sim 148 \sim$ 


\section{Chapter 8}

\section{Language and Words in an 'Asperger' World}

In order to analyse discourses used by participants, my observations of the filmed interviews focussed on varieties of discourses signalled in the language used within participants' narratives about their lives. Discursive language is best described by Foucault: "Descriptions are not language's faithfulness to its object, but the constantly renewed birth of an infinite relationship between words and things" (cited by Horrocks \& Jevtic, 1998, p.6). Discourses create habitual normalizations, and eventually, through socialization, change language. Whilst words chosen by participants existed as familiar meanings for them, word usage and meanings tended to change within narratives. An example is how concepts of ability were considered 'measurable' and applied like a spectrum; another example is how social considerations of normality, disability, stereotypes, categories and other classifications were used.

\section{Measurable 'asperger's' and talents}

Concepts of aspergers in the narratives tended to be measurable and tangible, in the sense that a person may have aspergers internally, and that there is a spectrum or scale of the perceived ability / functionality and independence / support needed. This illustrates the obvious, and in many instances, unavoidable dualisms created by the culture the participants live in. For example, participants understood that people not categorised as aspergers were those who do not exhibit behaviours or traits of the 
socially created 'syndrome'. These issues are often considered under medical terms, therefore are compared to social norm standards.

Conversely, those aspies deemed gifted tended to be measured against one another as successfully accommodating within social norms of nonaspies. In other words, the bench-mark for measuring giftedness by these participants tended to be how well someone was accepted and accommodated within regular society, as well as identifying as aspie. Hence, aspies deemed unsuccessful were those labelled medically as 'lower functioning' or 'more aspergers' than others.

When I questioned the choice of words used, participants would often confirm their descriptive words were considered by them as the conventional thinking surrounding the issue in their generation and culture. The normal or cultural understanding was often believed because it was expressed by medical professionals, written in books, and shared amongst aspies and other communities participants were involved in. Yet, as experiences of certain issues were diverse amongst participants, this indicates the powerful yet shifting nature of discourse. For example often words were used in a variety of ways in differing situations, such as the measurable idea of autism and aspergers, and whether aspie meant a pride of the identity, or a general membership of people labelled aspergers. One example follows:

Kay: like most people see a full picture when they look at something. Some people, it's more like 'join the dots', and experience helps build the ability to join the dots to make the functioning. And those people who are low functioning, they've got fewer dots and less ability to connect them up. 
But time and experience and a bit of detective work can quite often fill in the gaps and that can be on how people are motivated, uh, how they sort of operate

\section{Cam: you mentioned 'low functioning'?}

Kay: oh that's just from the... literature... [Thinks momentarily] Now it's not low functioning in terms of how he is, it's how he fits into what he wants to do, versus what his group wants him to be doing

Reflecting upon this excerpt, Kay described her understanding using everyday language other people use, within medical discourse (e.g. in her view the words 'low functioning' existed as a measured status that evolves with support from the environment). Kay is a self-identified aspie; like all participants, her descriptions of aspies tended to 'accept' or 'conform' to ways that other people from her social networks, (in her case, media and literature) describe the 'syndrome' and adopt some of the words and meanings, but she did add to this medicalised view a personal fresh perspective with pride.

On the other hand, when I questioned Kay about the use of the words 'low functioning', Kay replied casually that this is what is generally used in the literature. However, she changed her dialogue to possibly convey a more empathetic feel, by defining ability as a comparison to standards created by society, and then contrasted these with the wants or needs of the individual. In other words, the individual has a choice or perhaps a dilemma, of deciding what he/she wants to be positioned as, rather than being forced to reach a standard of a certain ability or skill that a majority of people judged as 'normal'. This focus on the aspie's own choices indicates Kay's attempt to add in aspects of a rights-based discourse. 
This is not easy, however. In the following example, Kay stated she cannot escape the terminology of commonly used descriptions of ability when referring to her son Gareth:

Kay: This is one of those areas where an awareness of 'expert' definition and labels is inescapable. To get access to appropriate education for my son's (gifted) needs he has to be labelled and it does help that the label has not yet been twisted negatively as the 'special' label has.... (son's name) is opposed to labels and isn't even happy about people needing to be named.

Unfortunately, it appears to be a complex process to create an alternative discourse given the predominantly strong medical discourses. The excerpt also demonstrates the dualistic nature that Foucault (1975) and others (Bagatell, 2007; Biklen \& Kliewer, 2006; Fulcher, 1989) believed is inevitable in evolving cultures. People are either with aspergers or not with aspergers; self identified as aspies, or simply labelled 'person with aspergers'; there may also be an assumed reference to scales of how 'much' a person has aspergers, such as seen in the Diagnostic Criteria for Autism Spectrum Disorder (Ministry of Health, 2008). Eion described how he met a child with diagnosed autism when he was a teenager, and how he developed an early understandings of a measurable criterion of autism and later, aspergers.

Eion: Well I had always known. When I was 18 I went to Handicamp and looked after a boy with classic autism called Dylan and Dylan was like me but further down that track [arm gesture a length] I... could see he was like aloof, closed off communication wise, and I was a little bit at that age as well

Cam: Sorry you said 'further down that track'?

Eion: Further down the spectrum [arm gesture] I just think of it as a spectrum, so I began referring myself as having 'semi autism' which is um doesn't exist, and it was only when my 
son was diagnosed by a paediatrician, at 3 years of age... he said "Luke, like his father, has aspergers syndrome". And having diagnosed thousands of people with aspergers syndrome I took it from him that that was correct... and I've since got diagnosis from an adult paediatrician but not a psychologist yet

This is another example of a predominantly medical discourse. 'Further down the track' indicates the idea that autism exists on spectrum of abilities and people are labelled according on the spectrum. Eion empathised with the child; like Kay, he understood that the child is somewhat the same and different because the child's judged abilities indicated a lesser estimation of ability, even within the aspie community. 'Semi autism' again reflects this worldview. This example also challenges the dualism that people are either autistic or non-autistic. Though this definition was given the subjective category aspergers years later, it is still believed to be on a spectrum of autism, rather than a dualistic non-autistic label.

Whilst the participants were proud to identify as aspies, they recognised the judgements of being positioned as 'more abled' aspies compared to other people with aspergers or autism. Hence they discursively continued to exercise the medically, and socially accepted language of the day.

\section{Stereotypical world}

The language used by participants also adds to and confirms the social identification of stereotypes. For example, there is a socially constructed idea that academically clever people who are considered inept, appearing awkward and with few friends, are a special group often casually labelled as 'geeks'. This is a label that may be placed upon those also categorized 
mathematics and science easy, but socialization challenging. Whilst the term is generally considered derogatory, some aspies adopted the term with a positive spin, devising the name 'geek syndrome'.

Kay: I usually get on fine with guys, especially as geeks and you know, computers and that sort of people

This is an example of playing with conventional and modern language use, and creating a label for shared understandings and identities. It challenges current ideas about being popular, being classified as aspergers and being inept as part of a social identity. This is to a point that disability is less in focus but rather a cultural spin to a social sub cultural category.

Furthermore, the use of 'geeks' and 'geek syndrome' is a phenomenon that portrays anyone who fits the stereotype, and is not limited to those labelled as having aspergers. In this way, Kay stressed her comfort with the geek label:

Kay I'm happier identifying as weird, as a geek, as someone different, than as someone with a disability who needs to be constrained or protected.

A characteristic of 'geekiness' was an idea compared by the participants' perceptions of being aspies. Language and words in narratives meant participants recognized themselves as influenced by what their social standings were in institutionalised cultures; in other words, by the way power is used in a cultural sense toward and by people within stereotypical groupings. For example, Kay described how different she felt compared to other girls in childhood:

Kay: Oh from an early age I also had an interest in science topics and, and like, instead of playing with Barbie dolls l'll be more 
(likely to) play with meccano and you know objects...l've often been the only girl, in say, a higher level science class...

Her dialogue shows her rejection of what she saw as sexist stereotypes about girls and Barbies. In her comments, she suggests that girls not only enjoy playing with dolls but are likely to be less successful in science classes. Whilst she recognized she was different, she mentioned that her experiences were ordinary in her life.

Kay: Well, l'm normal, it's everyone else...

Cam: you know that aspergers really influences people in completely different ways. How do you think it's influenced your life?

Kay: Well I can't separate that from anything else...

The concept of normality is constantly contrasted in Kay's talk between aspie and non-aspies. It appears comparisons and contrasts continue to be major facets to the lives of many participants. It adds to the dimension of what is 'normal' and perceptibly different, and the dualistic nature that humans inevitably engage in. Alan confronted this with his statement:

Alan: you know l'm not going to necessarily go through the same sorts of, uh, socialization processes that other people go through, you know... they start their work at $17 \ldots$ go through their (life) paths... I am 52... I mean I hear people are 52 and they're grandparents um I'm not a grandparent definitively... and they're either happily married and they're settled domestically. I am none of those I haven't even got continuous employment history...

The power of wanting to be the same as other people in their culture was huge, despite if their lives were deemed 'normal' by themselves or other people. 


\section{What is 'disabled'?}

Words such as 'disabled' were rarely used by participants, but like choices of many words, discourses changed within narratives. 'Disabled' may conjure meanings of being unable or the opposite of 'able' or 'enabled' as seen in medical, charity and lay discourses. As Biklen and Kliewer (2006) argued most people choose to not be described as 'impaired if the 'lower ability' meaning and social positioning were negated.

Another possible reason why participants did not use the word disabled as often as the terms aspies or aspergers is perhaps intellectual disability was viewed as not always associated with aspergers. Some unwritten dialogue that was uttered by Kay prior to data collection about people with aspergers is that aspies do not have an intellectual disability, but rather a 'social disability'.

On the odd occasion, however, data portrayed concepts of the word disabled. For example, the extract mentioned earlier had Kea described a young man with aspergers whose photo was placed in the local newspaper,

Kea: Well he doesn't look any - he doesn't even look disabled! This demonstrates that there is indeed a stereotype within Kea's discourse of what a disabled person or an aspie may appear like. Therefore, his experiences of aspergers, as compared to people without aspergers, may be a type of disability. Kea also described how he organised a diagnosis to confirm to his manager he is an aspie. 
Kea: I did it because I needed to keep my job and disclosure meant that HR had to legally take my disability into account, or at least be seen to be taking it into account.

Whilst his disability was seen as normal when he grew up in a family of other aspies, the social context later changed for Kea. Interaction between him and his co-workers about his characteristics of aspergers meant his skilled abilities were overlooked, and he felt he was viewed by some as having a disadvantage or disability first. When asked if teaching his workplace about aspergers changed the outcome of his relationships, he mentioned:

Kea: Two of the staff have children on the spectrum so their views were probably more positive. Maybe. [Thinks]. One of them tends to wear his son's disability a bit like a fashion accessory and a cross that he has (to) bear.

Again, a measurable diagnosis of aspergers is applied in this situation, as well as the dualistic nature of disability and normality. Furthermore, Kea adopted the language his workplace used, despite not approving or being comfortable of the same labels they placed upon him. He felt that one of his workmates used his son's disability as a tokenistic difference, furthering a dualism between normal and disabled. To describe this experience, Kea needed to use the same words, to speak the same language, in order for him to accommodate within his workplace world and hopefully be noticed as a person that could be skilled and gifted, even if viewed and labelled as 'disabled'.

\section{Continuing with categories, characters and classifications}

In this study, despite participants calling themselves aspie and hinting of a few nuances of a rights-based discourse, participants' narratives demonstrate that medical language continues to dominate their 
discussions about aspergers and giftedness. Because medical discourse added weight to what Fulcher (1989) identified as disability lay discourses and charity discourses ${ }^{15}$, these discourse 'types' are influential to words used within narratives. As a result, participants were unable to remove themselves wholly from medically influenced discourses of aspergers or giftedness into a more rights based way or thinking and being.

On the other hand, discourses change, including traditional medical discourses. Words participants adopted from one discourse type may have been used in other ways in another discourse. Choosing a discourse is individualistic and constantly evolving, that often people are not awareness of the ongoing changes to discourse.

This challenge - pitting medical and rights views - was highlighted as a continuing paradox in Chapters 2 and 3 . My findings suggest that participants found this aspect of their narratives the hardest to discuss. The idea of an entire discourse transformation conveys a dualistic nature between 'rights based' and 'medical' discourses of disability and aspergers. This is parallel to Bagatell's narrative study (2007). However due to the small sample of participants, this may not be generalisable. Despite years passing between narratives examined in my literature review and participatory narratives of this study, the interplay of having to 'fit in' with what society ordains as necessary is indeed a continuing challenge.

\footnotetext{
${ }^{15}$ see Chapter Two 'Foundations of a Journey'
} 
Participants seemed to believe in both the inevitable yet alterable nature of social categorisation and labels. In effect, labels cannot be avoided, as society uses many different processes to classify people into groups to specify different characteristics, needs and the like, for example to access special educational funding. On the other hand, social forces place the dualistic preferences of whether or not certain categories are negative (e.g. disability) or positive (giftedness) in certain arenas. Therefore, it is the social conditions that determine whether labels and categories are viewed highly or less worthy. 
$\sim 160 \sim$ 


\section{Chapter 9}

\section{Gifted 'Aspies'}

In this chapter, I focus on narratives about talent or giftedness. Participants considered that having a talent or gift was possession of a positive trait, a skill measured at an ability level compared to other ability levels in their socialisations. I explore why some gifts are considered useful or not by participants, and how participants felt their skills were judged by neuro-typical society. Some participants continued to be proud of being an aspie when discussing gifts; therefore a section of this chapter focuses on whether their giftedness is a separate characteristic to being labelled as having aspergers.

\section{“I am an 'aspie', but am I gifted?"}

All participants spoke less about being gifted compared to their lengthy discussions about their lives in being an aspie. In fact, what life was like as aspies were participants' primary topic, despite the fact that interview questions related equally to being an aspie and to giftedness identity formation. For example, Kea was very interested to take part as a participant. His concern however was that, while he openly identified as an aspie, he did not see himself as someone acquiring or possessing skill worthy of classifying as 'talented'. In particular, he struggled to view himself as a gifted person:

Cam: When did you first realize you had this gift or talent and how did it come up?

Kea: Well I actually wrestled with the question actually [laughs] 
Because, well, I thought what talent do I have? I'm quite a critic and like to evaluate things. I am visual. Early on when I was younger I had a near photographic memory.

Kea said he was challenged to think of what talents he had or gained; yet he seemed to think of many examples for when he was considered different for not achieving socially expected standards, such as professional tasks at his workplace. There may be many reasons for this. Perhaps a major influence was the feeling of being different to others in terms of having impairment (whether participants deemed aspergers as a social or intellectual impairment, if any) and the challenging impact this may had on socialisation and fitting into a dualistic culture of mainly neurotypical people. Being labelled as gifted or admitting to having talent appeared a more varied and subjective ideal for participants, as opposed as labelled 'aspergers'.

This is related to the notion that to have a talent or gift creates social admiration, by those within an asperger world and by people from a neurotypical world. The social positioning of a gifted and aspergers status ranged from visionary to hopeful or unlikely.

Another reason that participants may speak more about being aspie than being talented is perhaps the cultures they live in may place less emphasis on being gifted, and more on supporting those social considered as 'not accomplishing'. Whilst there are people who are celebrated or revered as successful, as stated in my literature review, essential attention tended to be given to, perhaps, 'under-performing' learners, such as 
funding for students not reaching age related milestones (Colangelo \& Davis, 2003).

Furthermore, some cultures may place a level of humility upon speaking about one's talents - to do so may be viewed as 'showing off' or boasting. Hence, these factors were taken into consideration when analysing the many worldviews surrounding abilities.

\section{“"My asperger's gives me abilities”}

The talents Kea, Alan, Kay and Eion knew other people perceived them as having were believed by participants as being caused by a 'natural' inherent trait of having aspergers. For example, Kea had viewed his 'near photogenic memory' as normal because his family also had aspergers and some of the same traits. Another example was after some discussion about how aspies were often working in 'smart' engineering industries, Kea stated about his own ability of understanding engineering,

Kea: It's kind of (as if), engineering was 'semi- hard-wired' into, into the psyche, uh... I am interested in bits of pieces...

The characteristic of being inquisitive of how machinery and 'bits and pieces' work in engineering may be considered by other people within Kea's world as a talent; but for Kea, it was viewed as part of an asperger genetic or intrinsic makeup. All participants had overtones of the concept 'aspergers makes me...' as if this skill was controlled by a belief of aspergers as an unexplained phenomenon. Alan and Eion described their experiences, as follows:

Alan: (Aspergers) has made me a very, very individual' individual... yeah it's made me a very individual individual. I have a friend who... says I have certain, what I would call 'singularities' uh, 
one is that I do like the use of language... 'Hyperlexia' where I just simply adore the texture of a word. Words have texture for me so to simply say the word is to evoke the texture and if you like the colour and smell of the word as well...It's not simply an abstract reality for me...yes it has texture rather like...cuddling a teddy bear or something, um to say a word like -[thinks] 'nonsingularity' is quite lovely

Eion: It's (aspergers) made me choose some rather unusual causes and rather unusual interests to back and to back them in a rather different way that other people might ...it's caused me to be first at some things rather than last to join in as well... It's also that I end up a trendsetter as well which is rather unusual for someone with aspergers

This idea that certain skills are seen as natural or intuitive, hints at a medical explanation of genetics because the skills feel 'normalised' and 'real' within their discourses. However, the participants seemed to understand that these traits, perhaps as part of an asperger 'make up', are measured as different to the majority of neuro-typical people; this is illustrated in Eion's use of the words 'unusual' to describe his traits, and Alan's description of himself as a "very individual individual". In effect, it could also explain why the other participant Kia (mentioned earlier) did not recognise his near photographic memory and inquisitiveness identity as types of gifts, because the skills were viewed by him as natural traits or normality in his life.

Participants felt they needed to be acknowledged by society for certain tasks in order to understand their social standing as having any talent. Often gifts had been acknowledged by other individuals in higher social positions, such as parents, teachers, career advisers, psychologists and employees in the participants' worlds. For example, Eion shared his experience: 
Eion: I can't remember when I learnt I had talents with languages. I think it became clearer when I got to about 11 years of age, when I won a spelling contest, and then a French speaking contest and I won a scholarship to college.

Cam: How did it make you feel when you won all these achievements?

Eion: I felt that it was like l'd been given some acknowledgement of what I already knew and for others to see as well

This excerpt showed Eion assumed he had a mastery of learning languages, and was proud his ability was measured highly, when compared to the success levels of his peers. This acknowledgement inspired him to learn other languages and he realised he had a potential to succeed.

Kay described her experience of understanding she was considered gifted by adults:

Kay: Any person sort of regards themselves as being normal in themselves, it's more about other people's reactions to them. And so from the time I was at kindergarten... my mother was told um, "you better start saving, she's going to university" right? "She's very bright she knows a lot"... you know, as a... three year old, using polysyllabic words....

Kay later emailed the following passage to me to describe her understanding of the last excerpt:

Kay: It's not that I think I have any particular 'gifts', but more a matter of recognising a category to which I may loosely belong, and other people seem to like using categories.

This quote shows Kay's belief in a traditional "category" to which she belongs. The fact that the word has a commonly understood meaning gives direction to a discursive analysis of the way this word works to help people to accommodate to 'normal' life. 
Similarly to Alan's love of textured words (which he described as 'hyperlexia') and Eion's success learning languages, Kay understood her talents were also to do with advanced language skills, particularly at a young age. However, she said this was 'normal' for her; despite other people in her society measured these skills as gifted.

The excerpts show that participants understand how neuro-typical people view giftedness, and which traits are deemed worthy. However cultural opinions of what is gifted may create concepts of 'gifted asperger' representations, or possible stereotypes. Participants had shared with me concepts and knowledge of people with aspergers that are considered by society as gifted, such as from literature (Williams, 1992; Grandin, 2007) and in popular media, (such as the movie Rain Man ${ }^{16}$ ). Kay's idea of people with aspergers is that they may be considered socially inept and labelled 'geeks'. Kea's perception is that people with aspergers are often eccentric engineers. Alan, Kay and Eion shared understanding that people with aspergers may have particularly strong memories and advanced language abilities.

Asperger representations in popular media or literature may also emphasise in cultures what 'talents' and 'gifts' are considered valuable and 'make' people geniuses. These possible stereotypes of people with aspergers and gifts may exist like social stereotypes of disability, and may

\footnotetext{
${ }^{16}$ Rain Man is a movie about a man who is diagnosed as autistic, and with savant syndrome. Although he was considered as having limited ability in day to day living (such as personal care), he was also considered as having a high ability in numeracy and memory skills. Rain Man was a celebrated movie that enabled autism to be viewed in a dualistic gifted light (Treffert, 2007).
} 
have influenced participants' thoughts and ideas of what constitutes giftedness. Kea, for example, enjoyed creating art and music, but did not view these skills as gifts compared to other aspies' talents.

Kea I'm not a mathematical asperger, um, you know [thinks] we're defined as various types ... I'm, l'm not a numbers person, uh, I'm a visual person... I don't know about my painting... l've got a reasonable musical ear and she (mother) says I'm good at singing so that's.... the interesting... the thing is I thought you were actually talking about

savants

As can be seen in Kea's comment, the word 'savants' was something he had thought about. Savant syndrome was mentioned as the archetype or epitome of 'aspie- genius' by Kea, Eion, and Alan. Yet they doubted they would reach this apparently superior level. This is interesting as these words developed in some participants' narratives when asked questions about giftedness. None of the interview questions actually mentioned the word 'savant'. Savant syndrome therefore appears to be a recognisable category with a socially high status within the discourses of talent employed in my participants' talk.

\section{Worthy abilities, talents and gifts}

All participants believed people with aspergers have skills that are normal or part of their everyday lives. Whether the skills have been considered valuable to themselves or from other people came with mixed responses. This is due to the discursive interplay of their skills and their environments; namely, the interaction of whether skills can be useful in education or a career.

Cam: So do you think their talents are usually recognized by other people? 
Eion: Um the ones with so-called 'useful talents' [laughs] uh, they get recognized but I meet very few of them, probably about just one or two

Like most participants, Eion perceived most aspies talents are not regarded as useful in certain social conditions, so sometimes the skills are left undeveloped. On the other hand, some participants have made concerted efforts to adopt a personal value of the skill, regardless of the little or limited encouragement given them. Eion enjoyed learning new languages and understanding computers. He decided to hold his abilities in high esteem because they are considered attractive skills in his social system. Alan also held a sense of pride for skills he developed:

Cam: What sort of experiences have you had with these talents?

Eion: The language part, it gives me a lot of fun as well as a lot of trouble. It affects my sense of humour. I love word play puzzles. (Aspergers) it makes me very talkative or hyperlexic. It makes me fall out with some people and fall in with others. It helps me to choose certain jobs and certain tasks that are more inclined towards language and the IT skills. Well, that's where I spend most of my working life, working in that area and, well that's because, a love affair with my computer still hasn't ended

Interestingly, Eion above mentioned that aspergers 'gave him' the useful characteristic hyperlexia, the same word and characteristic Alan described earlier. The fact that two different participants believe this trait is 'due to' aspergers, hints at a possibility that the trait is worthy of being considered gifted and also a useful skill for these aspies to have.

On the other hand, some skills explained as 'due to' aspergers are not always considered useful. Eion commented that aspergers makes socialisation harder with some people. Alan also shared problems with 
socialisation as he mentioned in the next excerpt. However, he chose to accept his difference and "just be me":

Alan: The other thing I (am) really good at is I remember lists and lists and lists of facts. All facts are very important for me

Cam: That must be very useful

Alan: No it isn't actually. In fact it's a handicap in many respects, because they weren't necessarily the facts that teachers or the social environment is interested in. So it tended to... [thinks] set... me apart... The problem is that... at that time when I was growing up...it wasn't really regarded as a social skill or attitude that you wanted, it was personally satisfying but it wasn't socially satisfying... it might be something that you could use as a party starter or nothing more than that. So I simply had to sort of, give yourself value to that or I don't get any value at all. So um, that's how I so of looked at it anyway... I had to see value I had, to reflect back on it, and go 'just be me'

Similarly to being 'veiled geniuses', gifts or talents often failed to be recognized, according to these participants, unless aspects of their unconventional asperger characteristics made them appear different. The examples above illustrate the differences as dualistically being positive or negative, depending on their social environments.

Some participants described how their skills have also gotten them into trouble, often stating their 'aspie' characteristics are partly to blame. For example, Kea had described earlier how his inquisitiveness was portrayed to his manager as 'unproductive' when he took apart machinery to discover its workings.

Cam: What are your views of people with giftedness and aspergers? Are they recognized for being gifted?

Kea: Sometimes they are, sometimes they're seen as skilled people who are naughty. 
As Kea's excerpt shows, aspergers is not always understood by many people in the participants' lives, and their behaviours are often misunderstood as some kind of misdemeanour. Participants felt that opportunities that would reveal hidden talents or prove worthy skills may have been masked by perceived incapability.

Kea also described how people with aspergers had a "skill set" that was often "strangely shaped" compared to more common neuro-typical set of skills. For Kea, this was most evident at his workplace. He had drawn a diagram to depict his feelings of how his "set of asperger skills" was not appreciated by his workmates (page 146). 


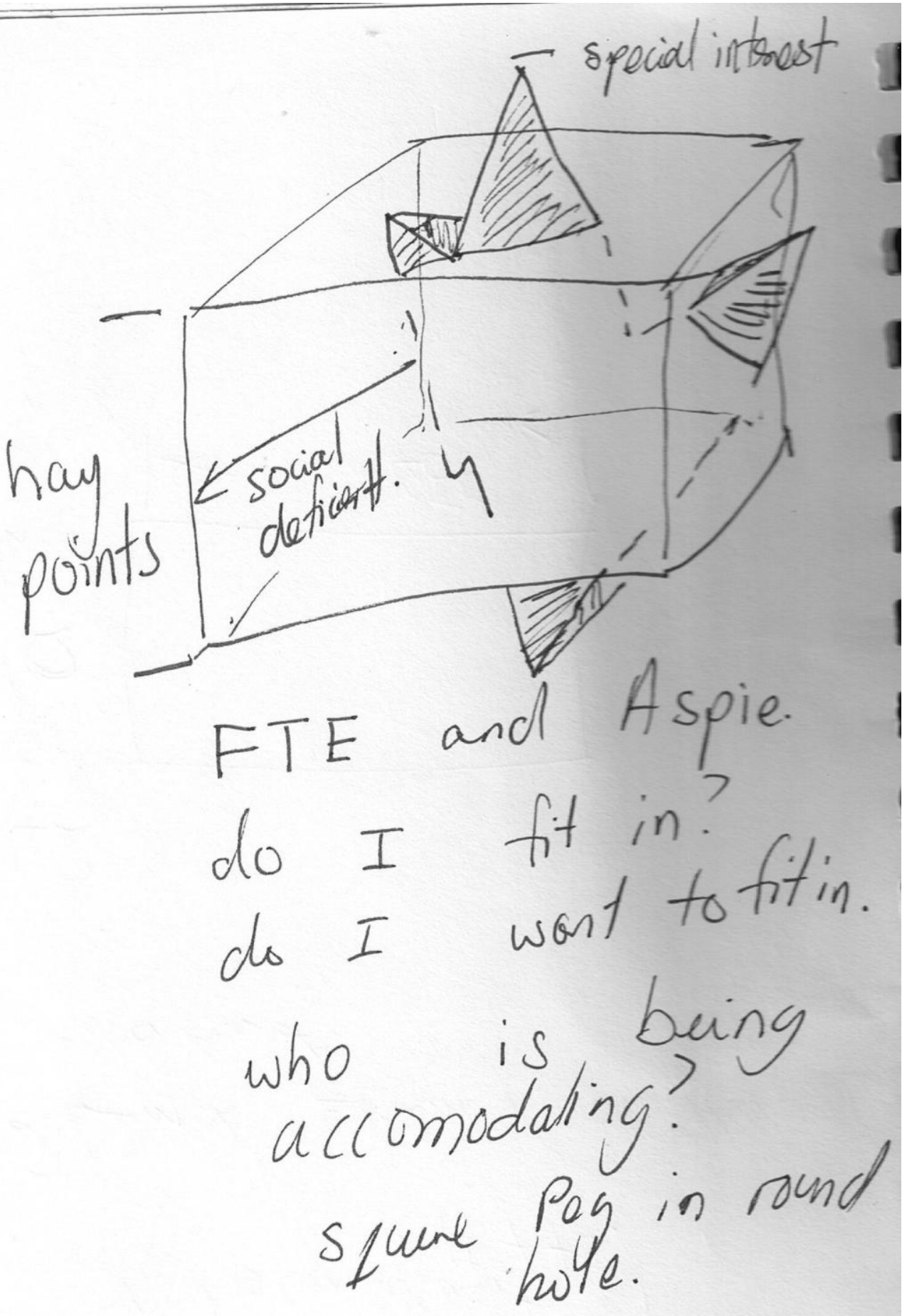

Kea's depiction of an "aspie skill set" at a workplace is based upon a human resource diagram used by Hays Group associates ${ }^{17}$.

${ }^{17}$ The Hay Group is a human resources management company. They use a cube to visually explain a business job description profile to measure compatibility and competency of an employee. Staff profiles are compared and contrasted and are used to predict a measured likelihood of employee success. Hay Points are awarded when an employee succeeds in expected and desired tasks of a job description. The cube is usually measured as a Full Time Equivalent role ('FTE'). 
Kea's depiction is that people with aspergers are likely to have social impairment that may result in a difficulty to do some tasks, such as being a 'team player'. Kea showed this concept marked with an arrow with the words 'social deficits' on the left side of the cube. This was not marked on all the cube's sides, as Kea believed people with aspergers are unlikely to meet this requirement and would receive less Hay points. The 'special interests' words and the prisms pointed outside the cube symbolise Kea's concept of gifts and talents in an asperger skill set. These may be advanced memory or inquisitiveness, skills Kea believed he had. However, Kea believed that people with aspergers have 'too much' of these abilities, and they may be a burden, hindrance and or unnecessary to a job well; therefore, the points are jaggedly 'outside' the desired job skill set.

Kea's written words 'Do I fit in?' and 'Do I want to fit in?' shows his confusion of whether or not to 'be himself' in his workplace, or risk being judged as deviant or incompetent. Perhaps a preference for him is for the workplace to accommodate to his unique skills. He described his experience as a 'square peg in round hole' as a metaphor that he had a 'weirdly shaped' skill set and does not match a job profile expected and socially moulded within his workplace. Hence, whilst Kea understood he had skills that are judged higher ability, they were not always considered worthy or useful. The metaphor is Kea's confusion of whether to accept social norms and accommodate, or dualistically, expect or hope the workplace to accommodate to him. 
As mentioned earlier, if people with aspergers reveal their challenges due to having aspergers, participants felt that people will see their disability first. This adds to the fact that data tended to reveal aspergers as a topic had a far greater focus in life experiences for participants than experiences of 'gifted talents'.

\section{Celebrating gifts}

Participants shared a belief that talents should be recognized in all people with aspergers, as Eion stated:

Eion: Develop it, share it with other aspies, take a risk and go to university and try to make it into a formal qualification somehow. Let it out [smiles]. Let us see it, stop hiding it away [smiles]. I keep finding people who keep hiding it away.

Eion shared with participants Kea, Kay and Alan three reasons other people with aspergers should be proud of gifts: firstly, representing a group of diverse people with diverse abilities and gifts; secondly, encouraging other people with aspergers to use their skills in ways that create relationships between themselves and neuro- typical people; and thirdly, people with aspergers could challenge social stereotypes of dualistic 'disabled aspies', words which conceptualise people incapable of being talented.

Participants were asked what sort of advice they would give other people with aspergers to celebrate talents. Participants spoke from past experiences of trying to use the talents at school, higher learning institutions and in a career. This could happen in a culture of empathetic support. Eion and Alan mentioned 'downplaying' the more obvious negated traits of being aspergers and using the talents to shine. 
Developing a personal value to the talent and being proud of it, is an aspect which participants believed made successful role models.

Eion: Oh share it (talents) with other people with aspergers syndrome. You know, let them see your talent and ask to see theirs.... it's not a normal social exchange but it is a social exchange and that's a good thing you know, it's good that we complement each other... because there may be others out there who are neurotypical and who have been uncomplimentary to us, and I know how much of, um, a beast it is for people with aspergers syndrome to have an extreme talent and to have no people recognize

In fact, participants sharing their talents during interviews developed a heightened sense of pride and worth, both within themselves and as a collective community. Whilst a high percentage of their descriptions of lives were about being challenged as an aspie, the conversations of their interests, successes and celebrations added great colour and excitement in an otherwise confusing world. 
$\sim 175 \sim$ 
$\sim 176 \sim$ 


\section{Chapter 10}

\section{Sharing an 'aspie' journey}

Participant narratives came 'straight from the heart' and were poignant and touching. Not only did these aspies admit to challenges of life experiences, in many ways they were reflecting and confronting the future of how they think, feel, live and share the identity of aspie, as is shown in the interview excerpts below

Discourses participants used tended to convey a dualistic nature for different concepts, such as the differences between people deemed to be aspergers and neurotypical people, or medical discourses and, perhaps, a rights-based discourse. Whilst medical and related discourses used in participants' talk have been criticised by many writers (e.g. Fulcher, 1989; Stockholder, 1994) as powerfully negative in their stance on aspergers, participants, in contrast, held a certain pride in being different. However this pride was demonstrated by participants as knowing the social rules or 'tricks' to accommodating within an often uncompromising or misunderstanding world. Deciding to position themselves as proud aspies therefore, took courage and understanding the possibilities of social positions

\section{Influences of discourses about 'aspergers'}

It is evident that medical discourse dominated other discourse types in the lives of participants. For example, discursive medical language and words continued to be habitually rooted narratives, indicating power dominance 
in culture generally. The use of words 'asperger syndrome' 'higher functioning' or 'social disability' were common examples, hence explains why participants lived the belief 'aspergers makes me...' as a reality and $\operatorname{truth}^{18}$.

However as will be demonstrated below, participants not only used language indicative of a medical discourse about aspergers, but used nuances hinting of related discourse categories, such as lay, charity and rights discourses ${ }^{19}$. Charity discourse was used when Kay said she preferred to be viewed by other people as a 'geek' rather than 'with

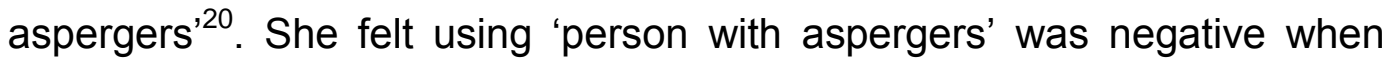
society used a charity discourse, and she would receive unwanted sympathy and be outed or ostracised.

Another discourse type I noted also influenced by the medical discourse was Lay discourse. An example of lay discourse was used by Kea when he stated that another aspie in the news 'doesn't even look disabled'21, hinting that people with aspergers have a disability and therefore have a stereotypical appearance. Another example was when participants spoke of the benefits and consequences of a diagnosis to confirm differences as perceived by them and people within their society. Lay discourse is evident when participants spoke of believing that a perception of giftedness is due to having aspergers as if an 'asperger mind' exists that

\footnotetext{
${ }^{18}$ See Chapter 9 'Gifted Aspies'

${ }^{19}$ See Fulcher (1989) in Chapter 2 'Foundations Before a Journey' - 'A Discursive Framework for Exploring the Asperger Journey - Categories of Disability Discourse' .

${ }^{20}$ See Chapter 8 'Language and Words in an Asperger World' - 'Stereotypical world'

${ }^{21}$ See chapter 8 'Language and Words in an Asperger World' - 'What is disability?'
} 
can be portrayed as intrinsically talented, useful and worthy of 'higher ability.

However, I felt that many participants had a sense of pride in being aspies that seemed linked to a rights based discourse. This is because participants wanted to be accepted rather than simply accommodated in an otherwise misunderstanding world, such as Kea's visual description of him feeling like a 'square peg in a round hole' in his workplace ${ }^{22}$. Some participants used a belief in being confident to make a decision regarding a social label and self identity, such as Kay stating "All of us (friends identified as aspies) dislike the $D=$ Disorder part and are not going to submit to testing for someone else's benefit"23. Kay's quote suggests rights based discourse as one way to be 'free' as an aspie, rather than constrained by classifications, judgements and stereotypes. This freedom was also felt when participants spoke of the strong relationships and shared understandings when socialising with other aspies.

I sensed that in order to 'fit in' with neuro-typical society, participants needed to adopt the same words, descriptions, behaviours and thoughts about aspergers used by people in everyday life. Discourses are so subtle in their operation that changing from one to an opposing discourse, such as including both medical and rights-based discourses, may be confusing when socialising with a majority of neuro-typical people. Another possibility is that participants may feel isolated or met with resistance when the

\footnotetext{
${ }^{22}$ See Chapter 9 'Gifted Aspies' - 'Worthy Abilities, Talents and Gifts'.

${ }^{23}$ See Chapter 6 'Aspie is Who I Am' - Diagnosis of Aspergers'
} 
majority of people do not understand the discursive words, beliefs and worldviews surrounding old and new discourses of aspergers.

\section{Living in a dualistic world}

Discourses tend to conceptualise aspects of life into dualisms. If something is the way it is, there is usually considered another, perhaps, 'opposite other' way to being. I felt this was a strong theme woven amongst the diverse narratives shared.

The most obvious dualism involved people who were considered (by participants) as having aspergers and those who were not. However, dualisms also existed for other opposites, even within considered autism and learning ability spectrums. Such dualisms carried discursive meanings, and tones of being 'one or the other', often with a positive or negative tone. The following dualisms were found in the data. Note the two lists are not representative of a positive or negative stance:

Neuro-typical /normal

Non-aspie

Aspergers / aspie

Not disabled / normal

Medical discourse of aspergers

Gifted ability

Gifted ability

Aspergers and gifts
Aspie / person with aspergers

Aspie

Autism / autistic spectrum

Disabled /impaired

Rights discourse

Disabled / low ability

Normal ability

Aspergers without gifts 
Narratives suggested that an ability spectrum tends to create conceptual measured normalities, with high or low abilities. Hence, there are more alternatives to 'ways of being' than simply the classifications listed above. Yet participants tended to focus on whether they are generally considered as being 'one or the other' of the above words in the table, in order to understand their social ranking. Their measured positioning became part of their realities. For example, participants chose to get a diagnosis to confirm if they were 'definitely aspergers'.

These discursively influenced behaviours forced participants to see their world as measured, compared, contrasted and categorised. This is parallel to data found in other studies of disability and giftedness, for example by excerpts of Parker (1992) and Ward \& Alar (2000). However, due to the ever-shifting social understandings of giftedness, talent, aspies, aspergers and 'ability' in general, dualisms are subjective and topical within the participants' paradigms. Hence, being considered aspergers and gifted today does not mean they will continue to use these labels eternally. Self identity may change according to the social forces of power and social institutions. Therefore, a person labelled aspergers and gifted today may find the label has a new description and meaning in another generation.

\section{Hide the 'aspie'... rather than pride of 'aspie'}

Participants' identities developed throughout their lives, helped by socializing within the communities with others who also identified as aspies. For examples, a sense of being part of a group allowed aspies to 'be themselves', share ideas, role model to each other and grow with 
support. On the other hand, participants often experienced ablest attitudes toward a perceived deficit concept of aspergers.

Decisions to hide or camouflage behaviours that may be judged as aspergers meant creating different identities that carried allowances for eccentricities. For example Kay identified with the stereotypical 'geek' $\left.\right|_{\text {abel }}{ }^{24}$. This identity may be judged negatively by a majority of society as a socially inept group, yet she felt the label was less ostracizing and less likely to be misunderstood as 'impairment'. Kay is similar to comments in narratives of other 'aspies' (e.g. Bissonnette, 2005, 2007; Ward \& Alar, $\left.2000^{25}\right)$.

However, hiding an identity of aspie, or 'downplaying' any asperger traits meant sacrifices and costs for participants. Participants, like people from previous studies, chose to mask their 'inner asperger' selves to enable any hope of acceptance and belonging to societies that may not understand their hidden differences (Coleman, 2001). For instance, Kay stressed that learning the 'social unwritten rules' was a beneficial way of surviving in a neuro-typical world ${ }^{26}$. However, this meant to participants as taking part in the same societies that participants argued do not entirely make accommodations for differences such as aspergers. For example, Kea indicated he would like to succeed at work ${ }^{27}$. However he stated that when his superiors told him he "should be happy to accommodate" into "their" neuro-typical world, he challenged that belief by answering "Uh- why?"

\footnotetext{
${ }^{24}$ See Chapter 8 'Language and Words in an asperger world' - 'Stereotypical world'.

25 See Chapter 4 'Life as We See It ' Gifted Aspies Talk About Their Lives'.

${ }^{26}$ Chapter 7 'Sharing the Asperger World as Aspies' - 'Learning how to survive socially'.

${ }^{27}$ Chapter 7 'Sharing the Asperger World as Aspies' - 'Learning how to survive socially'.
} 
with a perhaps defensive and offended response. Had Kea decided to conform in order to accommodate, he may have felt a need to 'hide' any unworthy asperger characteristics.

In many ways, participants hiding an aspie identity contradicted any statements of celebrating differences and individualities. The same could explain hiding self- perceived unworthy talents or gifts in the understanding that these are caused or due to aspergers; hence creating a perception of normalcy in order to live within the unwritten social rules. Kay's excerpts below describe this theme:

Kay I referred to myself as an Aspie, but high functioning (so much so that I can 'pretend to be normal') and you indicated that you were doing research on people like me. I think your written overview of your study included the phrase 'gifted people with aspergers'.

Despite Kay's choice to 'hide' her aspergers, she also used a rights based discourse when she stated her apparent pride of 'aspergers':

Kay: I guess I am old enough and secure enough that if anybody is going to get negatively affected by it - tough that's their outlook, not mine.

Participants felt traditional views surrounding autism and intellectual impairment in general were unfair, stereotypical, and hard to accept, let alone be proud of the self identity. This except is not other unlike personal narratives of 'aspies' (Grandin, 2007; Ward \& Alar, 2000). This is despite words participants in this study chose to use were fraught with a need to be accepted and accommodated within institutionalised society. 


\section{Positioning oneself amongst an 'aspie' and normal communities}

Social positions are created according to measured ability statuses. Narratives showed a much higher emotional proportion of the theme of how aspergers influences participant lives rather than achievements, successes and talents. Hence participants felt their social positions were as aspies with shared challenges in life, such as socialisation. This is parallel to other personal narratives and studies of aspergers. For example, Ward's narrative from my literature review showed a similarity to participant Alan, as both believed their talents are to do with memorization, 'caused' by their 'aspergers' (Ward \& Alar, 2000); however they also view these as more of a hindrance to socialization than value.

Ward: I can call up memories of things like cartoons so clearly that it is almost like playing a videocassette in my head. I used to do that and make the sound effects along with the story in my head. Other kids heard me making those noises and thought I was nuts. the thing I hated most in junior high was being teased. Mean kids used to try to upset me by imitating me or trying to get me in trouble. They thought it was funny that I was so "weird."

(Participant, Ward \& Alar, 2000, p.234).

Alan: I remember lists and lists and lists of facts - all facts are very important for me. It's a handicap in many respects because they weren't necessarily the facts that teachers or the social environment is interested in, so it tended to... set me apart...uh, it wasn't really regarded as a social skill or attitude that you wanted...

Using a lay discourse, emitting signs of ability would prove to others that the participants are 'higher functioning' than people labelled autistic that may be judged as not able to show similar ability levels. Participants often knew they were talented if someone had positively told them; this was the same for disability, which happened more often than celebrated talent. 
The above examples of how Ward and Alan were considered 'weird' for appearing different illustrate this point. Even so, people in society seem to need to seek evidence of competence, based upon their own worldviews of what this means. As Biklen and Kliewer (2006) wrote, "it is not in a state of unconsciousness or out of any belief in the autonomous individual that even in our own work invariably we feel compelled to somehow 'authenticate'..." (p.176). So, whilst labels are unavoidably required within society, this furthers the dualistic idea of being either talented and gifted or disabled.

Participants held strong viewpoints that humans are constantly pushed into categories and stereotypes, and the unfairness of negated social labels meant a possible life journey of confusion, loneliness, low confidence and confusion of self identify. Again, the unfairness was created due to the negative perception and focus of perceived disabilities. Few participants spoke of the rare positive experiences of being labelled gifted, as according to their perceptions of giftedness. Instead participants focussed on how socially considered talents or skills were well recognised in their "asperger skill set"

On the other hand, dualisms are subjective and could be challenged within the discourse participants used. Whilst all participants expressed opinions of being ostracised by society for perceived 'disabilities', Kea challenged the idea of dualism further:

${ }^{28}$ Chapter 9 'Gifted Aspies' - 'Worthy abilities, talents and gifts'. 
Kea: this whole dualism thing (is), we have this great need to 'fit' someone in to 'categorize' them, but the whole dualism thing is, that aspies fit in to neither 'gifted' or 'disabled' category. We kind of span it.

Kea felt somewhat angered at the need to categorise aspies as expected by neuro-typicals. However, he discursively continued to do so when he categorised other people as gifted or disabled. Conversely, he said he felt he is not a gifted person with aspergers, because he viewed this concept as a 'savant'. Similarly, Kay stated that she was not going to be diagnosed "for someone else's benefit", suggesting in her tone a personal entitlement, within a right based discursive slant. On the other hand, this decision does not prevent her from her idea of 'camouflaging' any sense of aspergers and instead 'pretend to be normal' in order to accommodate to an institutionalised normal society, the same society that aims to diagnose her.

The idea of 'dual exceptionality' raises the subjective issue of what exactly constitutes 'giftedness' and 'disability'. Many participants tended to view their talents as due to or caused by 'having aspergers', showing clear examples of lay discourse. This would mean then, that 'aspergers' may 'go hand in hand' with associated talents the participants' believed to have, such as advanced memory, mathematical and language skills. Similarly, Grandin stated,

All minds of the autism spectrum are detail-oriented, but how they specialize varies. By questioning many people both on and off the spectrum, I have learned that there are three different types of specialized thinking (in autistic people)

(Grandin, 2005, n.p). 
Whilst this may reinforce certain asperger stereotypes of 'geeks', it furthers the dualistic challenge of ability and disability, and remains the debatable theme of whether talent, skill, aspergers and impairment could well be intrinsic naturally.

This adds to an argument in favour of the gifted/disabled dualism, giving medical discourse a place to describe gifts that are common amongst people consider aspergers. In other words, the same medical or traditional descriptions of deficiency may be useful in some cases to describe traits that are considered talents. As Kay mentioned, people seem to prefer using labels and groups as part of their identities. Traditional discourses around disability and giftedness could be considered useful in this sense: perhaps the medical discourse surrounding Asperger's Syndrome may evolve over time to portray a less deficient discourse to one of difference and ability.

Another way of viewing the debate is that what is considered to be gifted in the participants' situations may well be because other (mainly, as participants believed, neuro-typical) people conveyed to the participants a normative definition of gifts. However, talent for these participants may not be the common skills such as memory or language acquisition; in fact, if 'advanced memory' is intrinsically 'normal' for people with aspergers, the standard measurement for this social group may be higher. Rather, talent for aspies in this study may be their measurements of successfully 'fitting into' society in ways that accept their different lives, and society showing an accommodating approval toward the participants (as opposed to the dualistically opposite of perception of inability). 
This would explain the participants' wish to support role modelling and sharing of experiences with others within an asperger community. Positioning themselves within this type of community becomes a real option once a status position is placed upon them, based on their perceived challenges, successes and gifts. When they have learnt the social rules to ensure society accommodates with them, (as opposed to 'learning how to survive' or 'hiding') participants may consider themselves as successful, and maybe even talented, in an asperger world.

This study is unique because it allowed participants to choose their identities prior to, and within their narratives. This opportunity meant that the descriptions based around their life experiences may be vastly different from research that is based on participants who were diagnosed prior to participant selection. As the research is also participatory, participants had opportunities to change their data or add further meaning after the filmed interviews. Because of this reason the participants' stories cannot be generalised to the wider aspie, asperger or gifted communities.

Perhaps additional research may evolve from participatory research of giftedness and aspergers. Participatory research is encouraged, as well as people with aspergers involved in decisions surrounding writing and promoting about aspergers and giftedness. More in-depth case studies are ideal for further exploring the gifted and asperger or aspie worlds in depth, whilst action research could have possibilities for enhancing participants' lives. Medical and qualitative research could play a part in 
future research of aspergers and giftedness also, particularly as this type of discourse remains a large focus of participants' talk. For example, medical or other common discourse could be slanted toward an objective look of academic and social results testing of gifted learners with aspergers at schools or tertiary providers and employment, or perhaps mental health statuses.

Societies discursively categorise and measure abilities, according to social understanding of worthiness, idealistic talents and measured social norms. Discourse enabled these concepts to be real in the participants' lives. Whilst they attempted to challenge the nature of their own and others' discourses, this placed larger challenges upon themselves as people already positioned within a larger society. 
$\sim 190 \sim$ 


\section{Chapter 11}

\section{Conclusion}

Narratives in this study showed that living in a world with both the labels of talented and aspergers was challenging and sometimes contradictory. Enabling participants to be co-researchers, meant that aspies had opportunities to reflect and give advice on research done about their lives labelled aspie, aspergers and gifted.

Aspies made clear their definitions of giftedness and disability have dualistically opposed meanings. As the review of literature in Chapter 2 showed, ability is measured according to social norms and expectations, creating a spectrum of ability levels and categories, of which 'people with asperger syndrome' is a social group.

However, comparisons to social norms inevitably change over time. Perhaps not surprisingly, the discourses used by participants in their talk changed during their narratives, particularly about explaining who they were, saying why they were labelled in certain ways, describing their social status positions and saying whether they preferred to accept the labels adopted by society or not. Acknowledgement of giftedness was an ideal measure of success within participants' worlds, and was superior to being labelled as anything else, namely, common dualistic terms of disabled, impaired or aspergers.

Participants' pride in an aspie identity seemed distinctly different to their pride in having talents. Participants felt the coined term 'aspies' seemed to 
strengthen the idea of their uniqueness as well as their sense of belonging within this group of similar others. Typical of any social group, such as medical professionals ${ }^{29}$, these aspies had created a shared language and understandings. For example, words such as 'neuro-typical' people, 'higher functioning' and 'asperger syndrome' were commonly used by participants. These aspies and other people with aspergers had also created social rankings within their group, such as aspies who can successfully assimilate into society as well as have talents that both people with aspergers and neuro-typical people deem worthy. This was demonstrated when savant syndrome was mentioned as the archetype or epitome of 'aspie- genius' by Kea, Eion, and Alan. When I mentioned a dual concept of aspergers and giftedness, Kea stated "the thing is I thought you were actually talking about savants"30.

Whilst Kay, Alan, Kea and Eion felt confident to identify as members within an aspie group, having displayed pride of talents meant participants felt less unlike the dualistic neuro-typical people - particularly because their societies recognized talents as worthwhile. This was comparative as talents to participants appeared to be about surviving in a neuro-typical world, and attempting to be normal; this was opposed to their pride of uncommon or unique abilities. In this sense, masked impairment and manifested talents were dualist endeavours in parts of their lives.

\footnotetext{
${ }^{29}$ See Chapter 4 "Life as We See It: Gifted Aspies' Perceptions of Life"

${ }^{30}$ Chapter 9 "Gifted Aspies" - "My aspergers gives me abilities".
} 
A sense of personal pride was obvious in the video recordings when participants recalled moments of being acknowledged to a skill they often already knew they had, such as Eion celebrating his language skills when a child. Participants' experiences of being discovered and celebrated by others in their asperger and neuro-typical worlds meant their skills being perceived as worthy talents by teachers, parents or peers. This is understandable, when compared to the limited literature of aspie or asperger narratives or research, which promote aspergers as a 'syndrome' and disability.

Skills Eion, Kay, Kea and Alan had, which other people perceived as talents were considered by participants as "part of my aspergers". They believed that the 'asperger make up' intrinsically has abilities that may be judged at a higher level than social norms, such as Eion's ability to learn languages. However, these abilities were judged by the participants as normal for them. This may suggest the linking of traditional medical view that people with aspergers have talents that are perhaps genetically inherited. Another argument is that talent within a traditionalist giftedness discourse is not the same as a discourse about talents expressed by these aspies. Often, talent to them may be measured by social acceptance, fitting in and successful accommodation being a proud aspie in a neurotypical world.

Participants shared understandings that being proud as an aspie meant knowing when to conceal differences. This is despite participants continuing to strive for asperger rights and supporting others living under 
this label. For example, self-diagnosed Kay happily admitted in her narrative she was an aspie, yet had not allowed her son or herself to be labelled so by other people. Perhaps this explained my difficulties in finding participants for this study. Many people who felt diagnosis confirmed they had aspergers did not want to discuss the nature of their lives, perhaps due to a disability stigma attached to having aspergers, and a want or need to 'fit in' with the rest of society. The often negated label of aspergers means some people may feel 'unsuccessful' in life, a word not normally associated with descriptions of talents and giftedness. Alan, for example, stated he has not had a "normal life path"; he gave examples that he had never had children or married, had not settled domestically nor had continuous employment ${ }^{31}$.

Generally, participants preferred to identify as aspie if society would accommodate and accept their differences; this is shown in Kea's handwritten words "FTE and Aspie. do I fit in? do I want to fit in. who is being accommodating? square peg in round hole" (words quoted as original). The feeling of society needing to accept and accommodate to participants' differences were especially felt as they experienced attempts to accommodate to 'normal' society.

However, there was in participants' talk words influenced by dominant medical discourse. Participants' acceptance of such labels as their own tended to be somewhat restricting and lead them to conform to a lower social positioning of disabled within a normative world. Participants often

${ }^{31}$ Chapter 8 "Language and Words in an Asperger World" - "What is disabled?" 
used the language of dominant norms, adopting the same language, behaviours and viewpoints as the majority of society. For example, labels surrounding mental illnesses were prevalent in all narratives when participants were diagnosed as having mental illness. The term 'asperger syndrome' had not yet existed in their worlds, or in the worlds of some of the participants' initial diagnosticians. These diagnoses acted as confirmations to participants that there existed descriptions and explanations of 'what was wrong with them' compared to the rest of society, hence enabling a difference to 'become real' for them, as seen in Chapter 6.

I was not completely sure why my participants continued to use the labels aspergers and aspie when sometimes they were so challenged to accept it themselves. Perhaps this shows the strength of the medical discourse, and participants continue the discourse and dualisms, along with their social worlds. Dominant discourses such as medical discourse become imposed and contribute to worldviews that in some ways they are 'inscribed'. Participants' decisions to hide their characteristics of aspergers hinted at decisions to accommodate to a society, despite pride of being aspies. Consequently, attempts of accommodating to the normal world may be easier if one accommodates to the acceptable medicalised view of one's life. Therefore, medical discourse has its place in the asperger world, if at the very least to describe and measure what is normal or not.

Participants did not criticise the medical discourses surrounding aspergers, with pejorative terms such as 'syndrome' 'impairment' or 
'disability'. To challenge traditional or current view points of aspergers being an impairment and disability that medical discourse purports, is tricky as it means challenging original concepts, behaviours, language and ways of being. Perhaps, an initiation to support an alternative non-medical type discourse, such as a celebration of a healthy diversity of bodies, abilities and talents, may be preferable for participants. Yet it also leaves people with consequential outcomes. For example, if Kea decided against diagnosis of aspergers, his workplace would not understand his different ways of working, and treat him as if he is misbehaving.

In a detached way, aspergers and giftedness are words and concepts, labels with meanings - yet not so simply. Foucault would argue that the meaning of words happens due to socially institutionalised constructs, seemingly 'naturally' as the employment of particular ideals and concepts of a discourse. Participants had been judged as different, and measured according to other medical discursive language - but with a stance they 'had something wrong' as compared to the rest of society. Before the term 'aspergers syndrome' was developed, the idea of it had not existed in the participants' realities. Their worlds existed in the form of a diagnostic criteria and comparisons to criteria and 'normality'. Since the creation of asperger syndrome, participants were about to be tested and confirmed of their difference, whether proudly or not.

This was a very small study, though it did provide considerable depth in the amount of detail participants brought to the discussion of their lives. The data cannot be considered generalisable to all people with aspergers 
or identified as aspies. The study was also limiting to the reason that some people with aspergers may have challenges communicating their experiences in such an arena, for example due to lack of confidence of their self identities, or physical impairment of memory or communication.

Very few studies have taken a participatory stance with an objective look upon giftedness with aspergers. This is often due to a portrayal of aspergers being an impairment or disability, associated with a 'lack of ability' to partake in participatory research. The fact that other research about aspergers as a 'syndrome' have generally used people already diagnosed as having aspergers shows a uniqueness of this study, and a possible difference to data. There is a need to further explore and reflect the discursive way of life people labelled as aspergers or identities of aspies live, and how they influence the relationships of ability - namely dualistic differences of disability and intellectual impairment with giftedness and talent.

Concepts of what we know today as aspergers and talent could be viewed as a different way of living and thinking, rather than the continuous deficit discourse of aspergers, and paradoxical discourse when talent is added. Shifting discourses surrounding aspergers and talent means a shifting of attitudes and understandings of ability within social worlds. This may lead to more opportunities for people with aspergers to shine with talents, rather than wear their label as a mask or failed 'veil'. A pride in being described as different or diverse may replace the label of aspergers, or 
their coined terms 'aspie' or 'geek', etymology may change to reflect a differently positive or diverse light.

Changing discourse of aspergers and talent may allow opportunities for participants to choose a new label as an identity, rather than be forced into a stereotypical group. Aspergers and talent may not be discursively viewed as dualistic opposites. Perhaps they would be viewed as going hand-in-hand, much as do disability and impairment currently within medical discourses.

More research is needed to understand the nature of what discourses are available for people labelled as gifted and aspergers. Future research could take the ideas explored here further. A realistic goal is to include aspie writers of the subject, alongside disability professionals (particularly those holding dominant positions within discourses, such as doctors) and the rest of society. Conference, journal papers and public events could promote new ideas such as participatory research, action research or new slants to medical discourse in quantitative research. This would paint a wide picture of discourses surrounding abilities, and may add more bright colour to some otherwise darker life journeys of those deemed 'with a difference' 
199 


\section{References}

Association of Gifted Children (2009). The New Zealand Association for Gifted Children Website. [Online]. Retrieved March 1, 2009, from http://www.giftedchildren.org.nz/

Autism New Zealand Inc (2004). Autism New Zealand Inc: Including Asperger Syndrome and related disorders. [Online]. Retrieved March 1, 2009, from http://www.autismnz.org.nzl

Autism Spectrum Disability Information Network: ASDIN. (2009). [Online]. Retrieved March 1, 2009, from http://www.asdin.org.nz/

Bagatell, N. (2007). Orchestrating voices: autism, identity and the power of discourse. Disability and Society 22(4), 413 - 426.

Baker, B. (2007). The apophasis of limits: genius, madness, and learning disability International Journal of Inclusive Education:1, 1 - 33.

Baum, S. M., Herbert, T.P., \& Renzulli, J.S. (1994). Reversing underachievement: stories of success. Educational Leadership 3(52), 48-52.

Bennet, C. E. (August 2002). Emotional Roller-Coaster: Stories from Mothers of Young Disabled Children. Massey University Research Fund Project. Massey University.

Bevan-Brown, J. (2004). Maori perspectives of autistic spectrum disorder Rangahau Matauranga Maori: Maori Education Research. Ministry of Education Maori Research Division. Department of Learning and Teaching (p.30). Wellington: Massey University College of Education..

Bevan-Brown, J., \& Carroll-Lind.J. (2008). Making assumptions vs. building relationships: lessons from a participatory action research project to identify effective practices for learners with autism spectrum disorder. Kairaranga: Weaving Educational Threads. Weaving Educational Practice 9 (Special edition), 22 - 31.

Bickenbach, J. E. (1994). Voluntary disabilities and everyday illnesses. In M. H. Rioux and M. Bach (Eds.). Disability Is Not Measles: New Research Directions and Paradigms (pp.55, 109 - 125). Ontario: L'Institut Roeher Institute.

Biklen, D., \& Kliewer, C. (2006). Constructing competence: autism, voice, and the 'disordered body'. International Journal of Inclusive Education, 10 (2-3), 10 -188.

Bissonnette, L. $(2005,2007)$ My Classic Life as an Artist: A Portrait of Larry Bissonnette. [Online]. Retrieved on May 8, 2008, from http://www.myclassiclifefilm.com/ 
Blacher, J. (2002, September). Twice-exceptional: learning disabled and gifted. Exceptional Parent Magazine, pp. 100-103.

Bourne, J. (2004). Twice exceptional: teaching gifted students with learning disabilities in a regular classroom. The New Zealand Journal of Gifted Education, 14(1). [Oniline].Retrieved September 9 , 2005, from http://www.giftedchildren.org.nz/apex/v14no1art04.php

Braggett, E. (1998). Gifted and talented children and their education. In Ashman, A. \& Elkins, J. Educating Children with Special Needs. Sydney: Prentice Hall of Australia, pp. 229-283.

Bruzzano-Ricci, C. (2005). Gifted/ADD? Or gifted / learning disabled? Removing the mask of frustration. Tall Poppies Magazine, the New Zealand Association for Gifted Children. [Online]. Retrieved September 7, 2005, from http://www.giftedchildren.org.nz/national/article3.php

Burman, E. (1991). What discourse is not. Philosophical Psychology, 4(3): $325-341$.

Cash, A. B. (1999). A profile of gifted individuals with autism: the twiceexceptional learner. Roeper Review, 22(1): 22 - 95.

Clark, T. (1995). Giftedness and autism - the application of savant / splinter skills in the autistic population through curriculum design. A longitudinal multiple replication case study. Power point presentation notes. University of New South Wales.

Classen, D. R. \& Classen, R. E. (1997). Mentoring the gifted and talented. In Colangelo, G.D. Handbook of Gifted Education, (pp.254267).Boston: Pearson Education.

Colangelo, N., \& Davis, G.A. (2003). Chapter 1 Introduction and overview. In Colangelo, G.D. Handbook of Gifted Education, (pp. 310).Boston: Pearson Education.

Coleman, M. R. (2001). Surviving or thriving? (Gifted boys with learning disabilities). In Gifted Child Today Magazine (2001, Summer). [Online]. Retrieved August 13, 2005, from, http://www.idonline.org/ld indepth/gt Id/surviving or thriving.html

Cousar, G. T. (2001). The case of Oliver Sacks. The Ethics of Neuroanthropology. Indiana: Indiana University.

Cowan, R., Stainthorp, R., Kapnogianni, S.,Anastasiou, M. (2004). The development of calendrical skills. Cognative Development, 2, 169 178. 
Cresswell, J. (1998). Qualitative inquiry and research design: Choosing among five traditions. CA: Sage Publications. Dalziel, H. L. (2001, April). The New Zealand Disability Strategy: Making a World of Difference Whakanui Oranga. Office of Disability Issues, (pp.4-35). Wellington: Ministry of Social Development.

Encyclopedia Britanica (2009). Madness and civilization: A history of insanity in the age of reason. (2009). Encyclopaedia Britannica. [Online]. Retrieved May 18, 2009, from http://www.britannica.com/EBchecked/topic/355900/Madness-andCivilization-A-History-of-Insanity-in-the-Age-of-Reason

Encyclopædia Britannica (2009). Mental age. (2009) Encyclopedia Britannica. [Online]. Retrieved August 23, 2009, from http://www.britannica.com/EBchecked/topic/375337/mental-age

Foucault, M. (1973). The Birth of the clinic: An Archeology of Medical Perception. (A. M. Sheridan-Smith trans). London: Tavistock,

Foucault, M. (1965). Madness and Civilization: A History of Insanity in the Age of Reason. (R. Howard trans). London: Tavistock,

Foucault, M. (1975). Discipline \& Punish: The Birth of the Prison. New York, Random House.

Fulcher, G. (1989). Disabling Policies? A Comparative Approach to Education Policy and Disability. East Sussex: The Falmer Press.

Gaffney, M. (2008). Participatory action research: an overview. What makes it tick? In Kairaranga: Weaving educational threads. Weaving educational practice, 9. (Special Edition), 9-14.

Gallaher, J. J. (2003). Issues and challenges in the education of gifted students. In Colangelo, G.D. Handbook of Gifted Education. Boston: Pearson Education. pp.11-23.

Goggin, G \& Newell, C. (2005). Imagining disability tomorrow. In Journal of Future Studies (10) 2, pp. 69-74.

Grandin, T. (2007). Dr. Temple Grandin's Web Page. [Online]. Colorado. Retrieved October 18, 2007, from http://www.grandin.com/

Hermon, S. (2002). Gifted students with disabilities. Gifted and Talented Students. Ministry of Education. [Online] Retrieved August 20, 2005, from http://www.tki.org.nz/r/gifted/disabilities/index e.php

Horrocks, C., \& Jevtic, Z. (1997). Foucault for Beginners. Cambridge: Allen \& Unwin. p.7.

Lamont, R. (2008). Learning from each other. Kairaranga: Weaving Educational threads. Weaving educational practice, 9. (Special Edition), 38 - 42. 
Lindquist, B., Carlsson, G., Persson, E.-K., \& Uvebrant, P. (2006).

Behavioural problems and autism in children with hydrocephalus. In European Child \& Adolescent Psychiatry, 15, 214-219

Lyons, V. \& Fitzgerald, M. (2005). Asperger Syndrome: A Gift or a Curse? New York: Nova Publishers.

MacFarlane, S. (2000). Gifted children with learning disabilities - a paradox for parents from Ministry of Education, Te Kete Ipurangi The Online Learning Centre. Retrieved September 19, 2005, from http://www.tki.org.nz/r/gifted/reading/theory/disabilities e.php

McHoul, A., \& Grace, W. (1993). A Foucault Primer-Discourse, Power and the Subject, (pp.21-22). Melbourne: Melbourne University Press.

McMullen, P. (2000). The gifted side of autism. Focus on Autism and Other Developmental Disabilities, 15 (4, 239.

Miller, L. K. (2005). What the savant syndrome can tell us about the nature and nurture of talent. Journal for the Education of the Gifted, 8. (3/4), $361-375$.

Minichiello, V., Aroni, R., Timewell, E., \& Alexander, L. (1991). In-depth Interviewing: Researching people, (p.128). (2nd Edition). Hong Kong: Longman Cheshire.

Ministry of Education. (2002). Gifted and talent students. Gifted students with disabilities. Te Kete Ipurangi the Online Learning Centre. Retrieved September 19, 2005, from http://www.tki.org.nz/r/gifted/initiatives e.php

Ministry of Health. (2008). Appendix four: diagnostic criteria for ASD (DSM-IV-TR and ICD-10). New Zealand Autism Spectrum Disorder Guidelines. Wellington: Ministry of Health.

Moltzen, R. (1995). Special abilities. In D. Fraser, R. Moltzen \& Ryba, K. (eds.) Learners with Special Needs in Aotearoa / New Zealand, (pp.307-340).Palmerston North :The Dunmore Press.

Morelock, M. J. \& Feldman, D. H. (2003). Chapter 35 Extreme precocity: prodigies, savants and children of extraordinary high IQ. In Colangelo, G.D. (ed.) Handbook of Gifted Education, (pp.455 469). Boston: Pearson Education.

O'Brien, P. (2005). Where to from here? Inclusive research. Paper presented at the conference NISLD Seminar Series Trinity College, Dublin. 
Office of Disability Issues (2009). Consultation with the disability sector. Wellington. 30 April 2001: Hon. Lianne Dalziel, Minister of Disability Issues. [Online]. Retrieved Septemeber 5, 2009, from http://www.odi.govt.nz/nzds/speeches/30apr2001-dalziel.html

Oliver, M. (1997) Emancipatory research: realistic goal or impossible dream? In Barnes, C. \& Mercer, G. (Eds.), Doing Disability Research, (pp.15-31). Leeds: The Disability Press.

Parker, I. (1992). Discovering discourses, tackling texts. Discourse Dynamics: Critical Analysis for Social and Individual Psychology. New York: Routledge.

Plank, A. (2005). Exclusive Interview with author Jerry Newport. WrongPlanet.net The Online Resource and Community for People with Asperger's Syndrome. [Online]. Retrieved September 5, 2007, from www.wrongplanet.net

Plomin, R. \& Price, T.S. (2003). The relationship between genetics and intelligence. In Colangelo, G.D. (ed.). Handbook of Gifted Education, (pp.113-121). Boston: Pearson Education.

Priestly, M. (1997). Who's research? A personal audit. In Barnes, C. \& Mercer, G. (Eds.), Doing Disability Research, (pp.88-107). Leeds: The Disability Press.

Ramcharan, P. \& Grant, G. (1994). Chapter 10 - Setting one agenda for empowering persons with a disadvantage within the research process. In Rioux, M.H.\& Bach, M. (eds). Disability Is Not Measles New Research Paradigms in Disability, (pp. 227 - 244).Ontario: L'Institut Roeher Institute.

Rapley, M., Ed. (2004). The Social Construction of Intellectual Disability. New York, Cambridge University Press.

Rattcliff, D. (2004). Video and audio media in qualitative research, Ring Surf Qualitative Research Net Ring. [Online]. Retrieved August 5, 2004, from http://don.ratcliffs.net/video/

Reis, S. M. \& Ruban, L. M. (2004). Chapter 8: Compensation strategies used by high-ability students with learning disabilities. In Students with Both Gifts and Learning Disabilities - Identification, Assessment and Outcomes, 25, 155 - 198.

Riley, T., Bevan-Brown, J., Bicknell, B., Carroll-Lind, J., \& Kearney, A. (2004). Gifted and Talented Education in New Zealand Schools. Wellington: Ministry of Education.

Rioux, M. H. (1994). Introduction. In Rioux, M. H. \& Bach, M. (eds.). Disability Is Not Measles : New Research Directions and Paradigms, (pp. 1 - 7).Ontario, L'Institut Roeher Institute. 
Sisk, D. (2003). Gifted with behavior disorders: marching to a different drummer. In Montgomery, D. (ed). Gifted and Talented Children with Special Educational Needs, (pp131 - 154). London, David Fulton Publishers.

Smith, L. (2008). Ethical principles in practice: evidence from participatory action research. Kairaranga: Weaving educational threads. Weaving educational practice, 9. (Special Edition), 16-21.

Stockholder, F. E. (1994). Chapter 7: Naming and renaming persons with intellectual disabilities. In Rioux, M.H \& Bach, M. (eds.)._Disability Is Not Measles: New Research Directions and Paradigms, (pp.153 179).Ontario, L'Institut Roeher Institute.

Synque. (2007). Changing minds: a short description of Foucault's ideas on self identity. In Foucault and Identity. [Online) Retrieved July 25, 2007, $\quad$ from http://changingminds.org/explanations/identity/foucault identity.htm

Tallent-Runnels, M. K. \& Layton, C. A. (2004). Chapter 7 Gifted adults with learning disabilities in post secondary settings. In Sternburg, R. J. \& Newman, T. M. (eds). Students with Both Gifts and Learning Disabilities - Identification, Assessment and Outcomes (25), (pp.131-154).New York: Kluwer Academic / Plenum Publishers.

Tammet, D. (2006). Born on a Blue Day: A Memoir of Aspergers and an Extraordinary Mind. London: Hodder and Stoughton.

Treffert, D.A. (2007). Savant syndrome - island of genius. Wisconson Medical Society. [Online]. Retrieved July 23, 2007.

Taylor, S. (2009). Gardner's multiple intelligences theory - G \& T Related Reading for Support for Schools and Teachers. [Online]. Retrieved 5 August, 2009, from http://www.tki.org.nz/r/gifted/reading/theory/gardner e.php

Ward, M. \& Alar, N. (2000 Winter). Being autistic is part of who I am. Focus on Autism and Other Developmental Disabilities, PRO-ED Journals. 232 - 235.

Williams, D. (1992). Donna Williams personal space. [Online]. Retrieved August 5, 2008, from www.donnawilliams.net.

Willig, C. (2001). Adventures in theory and method. Introducing Qualitative Research in Psychology, (p.49). London: Open University Press.

Zarb, G. (1997). Researching disability barriers. In Barnes, C. \& Mercer, G. (eds.). Doing Disability Research, (pp.49-66). Leeds: The Disability Press. 
$\sim 206 \sim$ 


\section{Appendices}

\section{List of Appendices}

Appendix One: Notice for Participants

Appendix Two: Information Pamphlet

Appendix Three: Consent Form

Appendix Four: Consent Form for Images

Appendix Five: Transcript Guide 


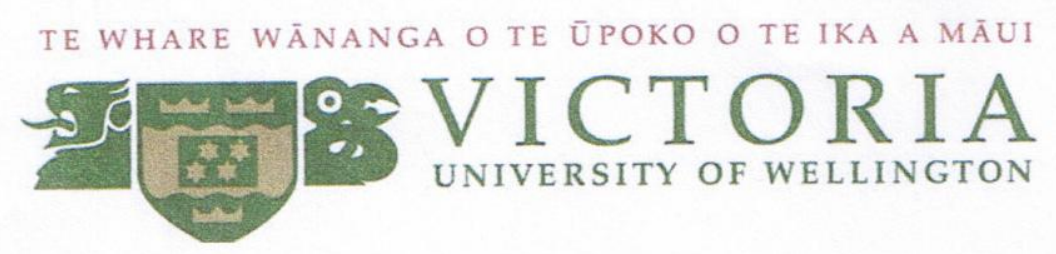

Notice for:

\section{"Gifted Individuals with Aspergers Talk About Their Lives"}

Kia ora! My name is Camellia Wong.

I am looking for some people to help me in a Victoria University of Wellington thesis project about what life is like for people with giftedness and Aspergers Syndrome.

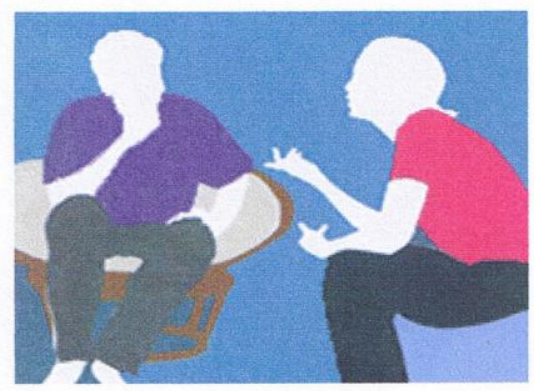

I am interested in working with people from all walks of life

Participants can be any age, but must be 16 years or over.

Gifts can be any kind of talent, e.g. mathematics, memory, creative, musical, sporting, that is done at a level higher than peers.

This study is a collaborative study, which means that participants have a chance to add to and help with the study.

All participants will receive movie vouchers for their time and commitment

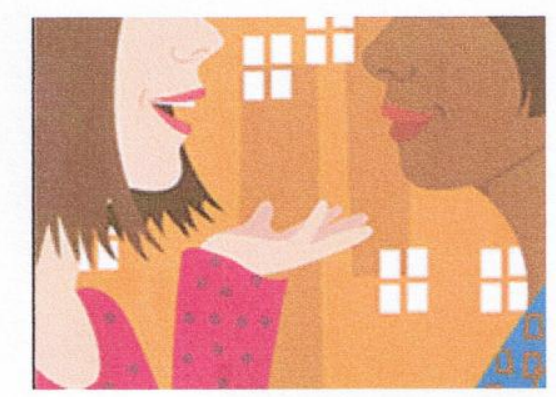

Interested? Contact Camellia Wong on: wongcame@student.vuw.ac.nz 
Appendix Two: Information Pamphlet for Participants

The following two pages are the front and back of the Information Pamphlet. 


\title{
Information sheet for a research project called
}

\author{
"Gifted Individuals with Aspergers
}

\author{
Talk About Their Lives"
}

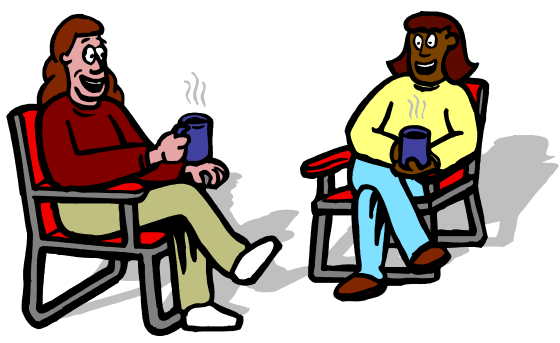

\section{Who is Involved?}

You have been invited to take part in this study if you identify as someone with Aspergers and who also has notable talents.

\section{Why is this study being done?}

This study is created to give gifted people who identify as having Aspergers the opportunity to share their views of what life is like with Aspergers and giftedness. It allows an opportunity for gifted people with Aspergers to take part collaboratively academic research, rather than just be participants. A benefit of the study is to educate and create awareness for the public of what life is like for people with Aspergers and giftedness.

\section{What is Giftedness?}

Whilst all individuals have skills and abilities, gifted people are described as having exceptional abilities that create both performance and the potential to achieve outstandingly well, in relation to peers. The giftedness may be in any areas of performance, for example, maths, music, memory, art and other unique talents.

\section{About the Researcher}

My name is Camellia Wong. I am doing a Masters thesis at Victoria University of Wellington. My supervisor is Dr. Lise Claiborne, who is Associate Professor of Educational Psychology at Victoria University of Wellington. She is helping me with the study. I have been a disability programme co-ordinator, support worker and advocate for people with diverse abilities. I am also involved in many disability settings in Wellington. I am interested in this topic because it is an understudied topic and I would like the chance to work with people who live with both giftedness and Aspergers Syndrome.

\section{What would you have to do?}

You and I will take part in a conversation

- We would meet and I would listen to your stories about different experiences of your life, such as social experiences, in education, relationships, careers. It would a conversation rather than an interview or question / answer session.

- The talk should last around 1 hour.

- I will record what you say, using a video camera. This will be so I remember what is said and how the conversation goes. It will be used in a way that will not distract how we talk to each other.

- This conversation will only be shown to you, my supervisor, and me.

- After the conversation, what you said on camera will be written into a transcript for future reference. We will watch the video and discuss it. We can watch the 
video conversation as many times as you need. This is where I will get my research findings.

- I may ask you questions to clarify about what you said in the interview, or make sure I don't miss anything out. You would help me make sure I understand everything correctly.

- You will have a chance to omit any information you share with me in the conversations prior to me writing up the study.

\title{
What would you get out of it?
}

You will have a chance to share what life is and was like for you living with Aspergers and giftedness. As this is a collaborative study, and you are a key spokesperson, your stories will add to the knowledge of what life is like for other people with giftedness and Aspergers. You may choose to keep a summary of the thesis or filmed conversation that you took part in.

\section{Confidentiality}

Everything you tell me is confidential. This research project has received approval from the Victoria University of Wellington Human Ethics Committee.

\section{For your safety:}

You may find talking about some experiences tricky, embarrassing or sad. Or there may be some things you don't feel comfortable to share with me, or on camera. That is fine, you don't have to. If you choose, you can have a supportive person attend parts 1 or 2. You can pull out of part 1 of the study at any time, without giving a reason, up until I analyse my findings. This will be two weeks after data collection.

At the end of the study:

I will keep the recorded conversations, transcripts and any other work securely for up to four years to write articles and give talks about the study. After four years they will be destroyed. You may choose to keep a summary of the thesis or filmed conversation that you took part in.

\section{What happens next?}

1. Take some time to think about being involved in the study as a co-researcher. Consider priorities, such as time involved, effort and other life commitments.

2. Consider confidentiality issues, personal life experiences and time availability.

3. If you decide to take part, we will have a discussion, and then you will be asked to sign a consent form.

4. We will arrange for a time to meet, and for the study to begin.

\section{Contact Details}

\author{
Reseacher: Camellia Wong \\ wongcame@student.vuw.ac.nz
}

Supervisor: Dr Lise Claiborne

lise.claiborne@vuw.ac.nz 


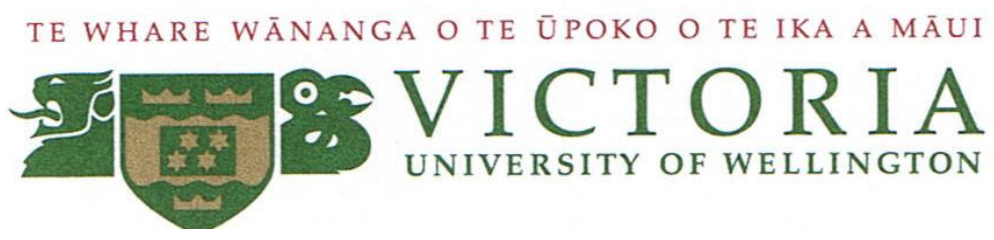

\title{
Consent Form to participate in a research study called:
}

\author{
"Gifted Individuals with Aspergers Talk About Their Lives"
}

\section{By Camellia Wong}

$\square$ I understand that the researcher and participants will be co-researchers in this study. This means that $I$ am able to ask questions, make suggestions and comments and have the opportunity to aid Camellia in her understanding of giftedness and Aspergers at any time of the study. This collaborative partnership has been explained to me and I am willing to take part.

I understand that the data will be collected from a conversation between Camellia and myself. It will be filmed with a video camera set up in the room.

I understand that only Camellia and her supervisor, Dr Lise Claiborne, will see the videotape. I am able to view with Camellia the videotape that I feature in.

I understand that my name will not be used. Instead a pseudonym will be used in the transcript that will be written up from the videotaped interview. These written transcripts will form the basis of the thesis.

I am able to make suggestions and comments to Camellia about the study prior, during and after data collection.

I am able to have a support person of my choice assist me in the duration of the study.

I understand that I can withdraw from this study up until the time Camellia starts to write her findings. This will be two weeks after the end of data collection.

Camellia will keep all recorded conversations, work and data findings securely up to four years after the study ends. After this, all data from the study shall be destroyed. I am able to have copies of data, recorded conversations if I choose.

Name of participant:

Signed:

Date: 
Email Address:

Address:

Signed.

Date

Camellia Wong, researcher 
Appendix Four: Consent Form for Images

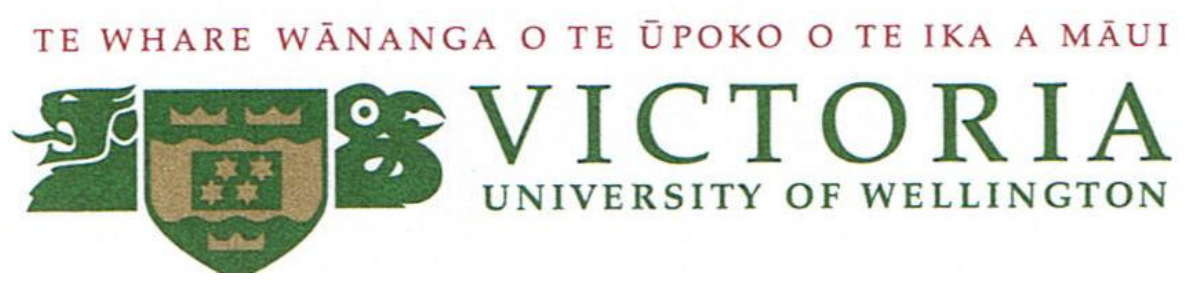

\section{Visual release form \\ Permission to use visuals / diagrams}

I hereby give permission for a visual / diagram /art work constructed and drawn by me, of my concept of:

An asperger identity applied to a business Hay's Point profile

To appear in the Victoria University of Wellington 'Gifted Aspies Talk About Their Lives' by Camellia Wong, to be published by in 2009 .

(participant)

(date) 


\section{Appendix Five: Transcript Guide}

All the quotations in this study come from raw data of transcribed interviews with Kay, Kea, Eion and Alan. The following is a guide to the presentation and understanding of the quotations.

Words in plain type

Words in italics

Single full stops and commas

[Pause 1 minute]

$[\ldots]$

[Laughs], [nods]
Participants' words.

Interviewer's words (myself).

Indicates pauses at end of sentences in participants' speech.

Indicates a length of time paused in participants' speech.

Indicates omitted speech by the author of thesis (myself) to shorten selected quotes from the transcript

Indicates action done by participant at the time of quote, as shown from filmed interview 